\title{
Colourful FKS subtraction
}

\author{
Stefano Frixione $e^{a, b, 1}$ \\ ${ }^{a}$ PH Department, TH Unit, CERN, \\ CH-1211 Geneva 23, Switzerland \\ ${ }^{b}$ ITPP, EPFL, \\ CH-1015 Lausanne, Switzerland \\ E-mail: Stefano.Frixione@cern.ch
}

ABSTRACT: I formulate in a colour-friendly way the FKS method for the computation of QCD cross sections at the next-to-leading order accuracy. This is achieved through the definition of subtraction terms for squared matrix elements, constructed with single colour-dressed or pairs of colour-ordered amplitudes. The latter approach relies on the use of colour flows, is exact to all orders in $N$, and is thus particularly suited to being organized as a systematic expansion in $1 / N$.

KeYwords: NLO Computations, QCD

ARXIV EPRINT: 1106.0155

\footnotetext{
${ }^{1}$ On leave of absence from INFN, Sezione di Genova, Italy.
} 


\section{Contents}

1 Introduction 1

2 Gluon amplitudes $\quad 4$

$\begin{array}{lll}2.1 & \text { Subtraction of colour-summed matrix elements } & 7\end{array}$

$\begin{array}{llr}2.2 & \text { Subtraction at fixed colour configurations } & 9\end{array}$

$\begin{array}{ll}2.3 \text { Subtraction at fixed flows } & 11\end{array}$

$\begin{array}{lll}2.3 .1 & \text { Fixed real flows } & 13\end{array}$

$\begin{array}{lll}\text { 2.3.2 } & \text { Fixed Born flows } & 18\end{array}$

3 Quark-gluon amplitudes $\quad 19$

3.1 Subtraction of colour-summed matrix elements 23

$\begin{array}{lll}3.2 & \text { Subtraction at fixed colour configurations } & 25\end{array}$

$\begin{array}{lll}3.3 & \text { Subtraction at fixed flows } & 27\end{array}$

$\begin{array}{lll}3.3 .1 & \text { Fixed real flows } & 28\end{array}$

3.3.2 Fixed Born flows 33

3.3.3 The $g \rightarrow q \bar{q}$ branching at fixed flows $\quad 34$

4 Summary of subtraction formulae $\quad 40$

5 Born-like contributions $\quad 41$

6 Discussion $\quad 43$

$\begin{array}{lll}7 & \text { Conclusions } & 47\end{array}$

$\begin{array}{ll}\text { A Conventions for colour matrices } & 48\end{array}$

B Colour operators, flows, and colour conservation $\quad 49$

$\begin{array}{ll}\text { B.1 Gluon amplitudes } & 49\end{array}$

$\begin{array}{lll}\text { B.2 Quark-gluon amplitudes } & 51\end{array}$

C A simple example: $0 \rightarrow u \bar{u} d \bar{d} g$

\section{Introduction}

The computation of amplitudes in QCD is a problem whose complexity grows factorially with the number of particles even at tree level. Such complexity stems from the extremely large number of Feynman diagrams that contribute to many-particle processes and which, apart from the inherent complication of the Lorentz structure, induce a proliferation of 
mutually-independent colour factors, that give rise to an involved colour algebra. Lorentz and colour structures can be separated by expressing scattering amplitudes as sums of products of dual (or colour-ordered) amplitudes times colour factors (see e.g. ref. [1] and references therein). The problem of the efficient computation of dual amplitudes has attracted considerable attention, and nowadays several solutions exist (Berends-Giele recursion relations [2], CSW relations [3], BCF recursion relations [4]). In order to predict observable cross sections, however, the colour algebra must be performed. Its factorial growth has been bypassed by working in the colour-configuration space [5], and by re-expressing it as an ordinary integral [6]; these approaches avoid the use of Feynman diagrams [7], and are essentially equivalent to colour-dressed recursion relations [8]; by these means, the Feynman-diagram factorial complexity is reduced to an exponential one.

The problem posed by the factorially-growing complexity of the colour algebra was simply irrelevant for the calculation of observables at the next-to-leading order (NLO) and beyond, because several other issues limited anyhow the applicability of NLO techniques to small-multiplicity processes. This is not the case any longer, thanks to the progress recently achieved in the automation of the two essential ingredients in an NLO computation: the subtraction of real-emission singularities [9-12] (where use has been made of the universal subtraction formalisms known as FKS [13, 14] and dipole [15]), and the computation of one-loop matrix elements [16-20] (based on generalized unitarity [21-23] and integrand reduction $[19,24,25]$ techniques). Therefore, the question becomes relevant of how to best organize an NLO computation, in order to be able to exploit the solutions that work well for tree-level matrix elements, and to use new tree-level approaches which may become available in the future. As far as the one-loop contribution is concerned, the problem is conceptually analogous to that relevant to tree-level amplitudes (but technically more complicated, see e.g. ref. [26]), since it consists in finding the optimal representation of an amplitude in terms of dual amplitudes and colour structures. On the other hand, the subtraction of real-emission singularities is complicated by the fact that it must be performed at the level of amplitudes squared, while it is the amplitudes (not squared) that display the simplest factorization properties in the soft and collinear regions (see e.g. ref [27]). As a result, in the subtraction terms defined in the FKS and dipole formalisms, the colour structure is not factorized, since it appears in both the universal kernels and the short-distance, process-dependent reduced matrix elements. Such a convolution is avoided in the antenna subtraction method [27-29], which is based on the use of the squares of, or the interferences between, dual amplitudes as elementary quantities whose singularities have to be subtracted.

The aim of this paper is that of formulating the FKS subtraction in terms of colourdressed and colour-ordered amplitudes. This will allow one to use, with only a few trivial modifications, the techniques developed at tree level to deal with the problem of the factorially-growing complexity. In order to be more precise, let me briefly digress and introduce the tenets of the FKS method.

The basic idea of the FKS subtraction formalism is that of treating in an independent manner the singularities present in a multi-parton matrix element squared. This is achieved by damping all singularities except one soft and one collinear, which are pre-determined, 
and by repeating this procedure for all singularities in turn. One introduces a set of functions $\mathcal{S}_{i j}$ such that:

$$
\sum_{i j} \mathcal{S}_{i j}=1
$$

The sum in eq. (1.1) can be thought of as extending to all strongly-interacting pairs of particles, although in practice significant simplifications are possible (see ref. [11] for an exhaustive discussion). For a given $(i, j)$ pair, the function $\mathcal{S}_{i j}$ is equal to zero in all soft and collinear limits, except those associated with $k_{i} \rightarrow 0$, and with $k_{i} \| k_{j}$. Given a real-emission matrix element squared $\mathcal{M}$, one exploits eq. (1.1) by writing

$$
\mathcal{M}=\sum_{i j} \mathcal{M}_{i j}, \quad \mathcal{M}_{i j}=\mathcal{S}_{i j} \mathcal{M}
$$

The matrix elements $\mathcal{M}_{i j}$ are independent from each other and, thanks to the properties of the $\mathcal{S}_{i j}$ functions, have one soft and one collinear singularity at most (depending on the identities of particles $i$ and $j$ ). These singularities are subtracted as follows: be $E_{i}$ and $\theta_{i j}$ the energy of parton $i$ and the angle between partons $i$ and $j$, in the rest frame of the incoming particles. The (divergent) integration of $\mathcal{M}_{i j}$ over the phase-space $d \phi$ is replaced by its (convergent) subtracted form:

$$
\mathcal{M}_{i j} d \phi \longrightarrow\left(\frac{1}{E_{i}}\right)_{+}\left(\frac{1}{1-\cos \theta_{i j}}\right)_{+}\left[E_{i}^{2}\left(1-\cos \theta_{i j}\right) \mathcal{M}_{i j}\right] \frac{d \phi}{E_{i}}
$$

If one neglects, for the sake of this discussion, the factors that multiply $\mathcal{M}$ in the square brackets on the r.h.s. of eq. (1.3), one sees that the result of the two plus prescriptions is that of constructing the linear combination:

$$
\mathcal{M}-\mathcal{M}_{\mathrm{SOFT}}-\mathcal{M}_{\mathrm{COLL}}+\mathcal{M}_{\mathrm{SC}}
$$

Here, the second, third, and fourth terms are the limits (in the sense of asymptotic behaviour, as customary) of $\mathcal{M}$ when $E_{i} \rightarrow 0, \theta_{i j} \rightarrow 0$, and $\left(E_{i}, \theta_{i j}\right) \rightarrow(0,0)$, respectively.

As was already mentioned, so far the FKS procedure has been formulated by assuming that all contributions to eq. (1.4) be colour-summed. In the present paper, $\mathcal{M}$ will be decomposed into a sum of terms, each of which has an immediate interpretation in terms of colours. Furthermore, this sum will have to commute with the subtraction procedure. In other words, each of the terms in the sum must have well-defined soft, collinear, and soft-collinear limits, so as for each such individual term a linear combination identical to that of eq. (1.4) can be defined, which is finite locally in the phase space. I shall consider two options: the sum over colour configurations, and the sum over colour flows. In the former case, the relevant matrix elements will be the colour-dressed amplitudes squared; in the latter case, they will be the squares of, or the interferences between, colour-ordered amplitudes.

This paper is organized as follows. In sections 2 and 3 I shall carry out the programme described above for pure-gluon amplitudes and for generic quark-gluon amplitudes respectively. The reader who is not interested in the technical details of the derivation may 
skip these two sections, and go directly to section 4, where I summarize the results for the limits of the matrix elements. In section 5 I consider the case of the contributions to the NLO cross section which do not originate from the subtraction procedure of eq. (1.3). Section 6 presents a discussion on the findings of this paper and a very brief comparison with antenna and dipole methods, and section 7 reports my conclusions. The conventions adopted for the colour matrices are given in appendix A, while appendix B contains a few technicalities, whose role is important for the understanding of the derivations presented in the main text. Finally, in appendix $\mathrm{C}$ some of the findings of this paper are applied to a quark-gluon process.

\section{Gluon amplitudes}

Given that the aim of this paper is the re-organization of the subtraction procedure in a colour-friendly way, it is convenient to consider all particles entering a hard scattering as outgoing, in order to simplify the notation. The Born and real-emission processes will therefore be

$$
\begin{aligned}
& 0 \longrightarrow n, \\
& 0 \longrightarrow n+1,
\end{aligned}
$$

respectively. Furthermore, it is not restrictive to assign labels $n+1$ and $n$ to the FKS parton and to its sister, respectively. ${ }^{1}$ The final results which will be obtained here will be easily cast into the usual form adopted in the context of FKS subtraction elsewhere $[11,13,14]$ — processes with a physical four-momentum configuration $2 \rightarrow n-2$ or $2 \rightarrow n+1-2$ will be obtained by crossing, while partons (and in particular the FKS parton and its sister) may be relabeled. In the introductory part of this section, where I shall discuss the general features of the scattering amplitudes, I shall consider the Born process of eq. (2.1) to be definite. The case of the real-emission process of eq. (2.2) can simply be obtained with the formal replacement $n \rightarrow n+1$.

Given an $n$-gluon colour configuration in $\mathrm{SU}(N)$

$$
\left\{a_{i}\right\}_{i=1}^{n}, \quad a_{i} \in\left\{1, \ldots N^{2}-1\right\}
$$

the corresponding scattering amplitude can be written as follows, where I adopt the representation used in ref. [1]:

$$
\begin{aligned}
\mathcal{A}^{(n)}\left(a_{1}, \ldots a_{n}\right) & =\sum_{\sigma \in P_{n}^{\prime}} \operatorname{Tr}\left(\lambda^{a_{\sigma(1)}} \ldots \lambda^{a_{\sigma(n)}}\right) \widehat{\mathcal{A}}^{(n)}(\sigma(1), \ldots \sigma(n)) \\
& \equiv \sum_{\sigma \in P_{n}^{\prime}} \Lambda\left(\left\{a_{i}\right\}, \sigma\right) \widehat{\mathcal{A}}^{(n)}(\sigma)
\end{aligned}
$$

\footnotetext{
${ }^{1}$ I remind the reader that, within one given region of the FKS dynamic phase-space partition achieved by means of eq. (1.1), the FKS parton is defined to be the only one that can give rise to soft singularities (hence, $i$ ), while the pair composed of the FKS parton and its sister is the only pair that can give rise to collinear singularities (hence, $(i, j))$.
} 
where I have introduced the shorthand notation:

$$
\Lambda\left(\left\{a_{i}\right\}, \sigma\right)=\operatorname{Tr}\left(\lambda^{a_{\sigma(1)}} \ldots \lambda^{a_{\sigma(n)}}\right) .
$$

When no ambiguity is possible, the first argument of $\Lambda$ (i.e., the set of colour indices) will be dropped. Here, $\lambda^{a}$ are the Gell-Mann matrices, whose normalization conventions are given in appendix $\mathrm{A}$, and $P_{n}^{\prime}$ is the set of non-cyclic permutations of the first $n$ integers. The non-cyclicity condition can be imposed by simply requiring

$$
\sigma(1)=1 .
$$

The quantity on the 1.h.s. of eq. (2.4) is the colour-dressed amplitude, and $\widehat{\mathcal{A}}^{(n)}$ is the dual (or colour-ordered) amplitude. Each term in the sum on the r.h.s. of eq. (2.4) corresponds to a colour flow (in which the colour of gluon $\sigma(i)$ is connected with the anticolour of gluon $\sigma(i+1))$. In a technical sense, I shall identify the flow with the ordered set

$$
(\sigma(1), \ldots \sigma(n))
$$

It will be convenient to regard each colour configuration as a vector $\left|a_{1}, \ldots a_{n}\right\rangle$; the set of all colour configurations can therefore be made equivalent to an ortho-normal base in a vector space (called colour space henceforth):

$$
\begin{aligned}
\left\langle b_{1}, \ldots b_{n} \mid a_{1}, \ldots a_{n}\right\rangle & =\prod_{i=1}^{n} \delta_{a_{i} b_{i}}, \\
\sum_{\left\{a_{i}\right\}_{i=1}^{n}}\left|a_{1}, \ldots a_{n}\right\rangle\left\langle a_{1}, \ldots a_{n}\right| & =I .
\end{aligned}
$$

Equation (2.4) suggests to define the following vectors in the colour space:

$$
\begin{aligned}
\left|\mathcal{A}^{(n)}\left(a_{1}, \ldots a_{n}\right)\right\rangle & =\mathcal{A}^{(n)}\left(a_{1}, \ldots a_{n}\right)\left|a_{1}, \ldots a_{n}\right\rangle, \\
\left|\mathcal{A}^{(n)}(\sigma)\right\rangle & =\sum_{\left\{a_{i}\right\}_{i=1}^{n}} \Lambda\left(\left\{a_{i}\right\}, \sigma\right) \widehat{\mathcal{A}}^{(n)}(\sigma)\left|a_{1}, \ldots a_{n}\right\rangle,
\end{aligned}
$$

which can be used to construct a vector that corresponds to the physical amplitude (i.e. the amplitude obtained by summing over all colour configurations or flows):

$$
\begin{aligned}
\left|\mathcal{A}^{(n)}\right\rangle & =\sum_{\left\{a_{i}\right\}_{i=1}^{n}}\left|\mathcal{A}^{(n)}\left(a_{1}, \ldots a_{n}\right)\right\rangle, \\
\left|\mathcal{A}^{(n)}\right\rangle & =\sum_{\sigma \in P_{n}^{\prime}}\left|\mathcal{A}^{(n)}(\sigma)\right\rangle .
\end{aligned}
$$

What is relevant to cross-section computations is the amplitude squared: ${ }^{2}$

$$
\mathcal{M}^{(n)}=\left\langle\mathcal{A}^{(n)} \mid \mathcal{A}^{(n)}\right\rangle
$$

\footnotetext{
${ }^{2}$ In refs. $[11,13,14]$, the notation $\mathcal{M}^{(n)}$ is used for an amplitude squared, times the flux factor, times spin and colour average factors. All these factors are omitted here, being irrelevant for the present discussion.
} 
Equations (2.12) and (2.13) can be used to rewrite the amplitude squared in two different ways:

$$
\begin{aligned}
\mathcal{M}^{(n)} & =\sum_{\left\{a_{i}\right\}_{i=1}^{n}} \mathcal{M}^{(n)}\left(a_{1}, \ldots a_{n}\right) \\
& =\sum_{\sigma, \sigma^{\prime} \in P_{n}^{\prime}} \mathcal{M}^{(n)}\left(\sigma^{\prime}, \sigma\right)
\end{aligned}
$$

where I defined

$$
\begin{aligned}
\mathcal{M}^{(n)}\left(a_{1}, \ldots a_{n}\right) & =\left\langle\mathcal{A}^{(n)}\left(a_{1}, \ldots a_{n}\right) \mid \mathcal{A}^{(n)}\left(a_{1}, \ldots a_{n}\right)\right\rangle \\
& \equiv\left|\mathcal{A}^{(n)}\left(a_{1}, \ldots a_{n}\right)\right|^{2} \\
\mathcal{M}^{(n)}\left(\sigma^{\prime}, \sigma\right) & =\left\langle\mathcal{A}^{(n)}\left(\sigma^{\prime}\right) \mid \mathcal{A}^{(n)}(\sigma)\right\rangle
\end{aligned}
$$

In terms of scalar quantities, eq. (2.19) is:

$$
\mathcal{M}^{(n)}\left(\sigma^{\prime}, \sigma\right)=\widehat{\mathcal{A}}^{(n)}\left(\sigma^{\prime}\right)^{\star} C\left(\sigma^{\prime}, \sigma\right) \widehat{\mathcal{A}}^{(n)}(\sigma),
$$

where I defined the colour-flow matrix element:

$$
C\left(\sigma^{\prime}, \sigma\right)=\sum_{\left\{a_{i}\right\}_{i=1}^{n}} \Lambda\left(\left\{a_{i}\right\}, \sigma^{\prime}\right)^{\star} \Lambda\left(\left\{a_{i}\right\}, \sigma\right) .
$$

The hermiticity of the Gell-Mann matrices and eq. (A.5) imply that $C$ is a real, symmetric matrix:

$$
C\left(\sigma, \sigma^{\prime}\right) \in \mathbb{R}, \quad C\left(\sigma^{\prime}, \sigma\right)=C\left(\sigma, \sigma^{\prime}\right) .
$$

Note that while the amplitudes at fixed colour configurations are orthogonal (a condition which is formally enforced here by eq. (2.8)), this is only true for $\left|\mathcal{A}^{(n)}(\sigma)\right\rangle$ in the large- $N$ limit. In fact, it is well known that

$$
\mathcal{M}^{(n)}\left(\sigma^{\prime}, \sigma\right)=c_{\sigma} \delta_{\sigma^{\prime} \sigma} N^{n-2}\left(N^{2}-1\right)+\mathcal{O}\left(\frac{1}{N^{2}}\right)
$$

with $c_{\sigma}$ a suitable real number. From the definition, one also sees that

$$
\mathcal{M}^{(n)}\left(\sigma^{\prime}, \sigma\right)=\mathcal{M}^{(n)}\left(\sigma, \sigma^{\prime}\right)^{\star}
$$

The quantities defined in eqs. (2.17) and (2.19), or their analogues for quark-gluon amplitudes to be introduced later, will be the basic building blocks for the definition of FKS subtraction at fixed colour configurations and flows respectively. As far as the latter is concerned, a final comment is in order. Colour flows are an important ingredient in the context of event generators, where they are used to determine, on statistical basis, the colour connections amongst the hard partons which initiate the showers. This determination is driven by $\mathcal{M}^{(n)}\left(\sigma^{\prime}, \sigma\right)$ with $\sigma^{\prime}=\sigma$ (since such quantities are positive-definite), while the matrix elements with $\sigma^{\prime} \neq \sigma$ are taken into account only in an averaged sense [30]. Therefore, as far as event generators go, a flow may actually be better defined as the pair of 
identical permutations $(\sigma, \sigma)$, the pair understanding a quantity relevant to the amplitudesquared level. In order to generalize this idea in a way consistent with the terminology typically used when dealing with amplitudes, I shall call the pair

$$
\left(\sigma^{\prime}, \sigma\right)
$$

a closed flow. This reminds one of the fact that the pair in eq. (2.25) corresponds to a set of colour loops; the counting of loops is a very simple way to estimate the largest possible power of $N$ which appears in $\mathcal{M}^{(n)}\left(\sigma^{\prime}, \sigma\right)$. To further this, as one sees from eq. (2.21) the two flows that define a closed flow play a similar, but not identical, role. When I shall need to distinguish them, I shall call the ordered sets

$$
\left(\sigma^{\prime}(1), \ldots \sigma^{\prime}(n)\right), \quad(\sigma(1), \ldots \sigma(n))
$$

as L-flow and R-flow respectively, with the understanding that they enter the amplitudes $\left\langle\mathcal{A}^{(n)}\left(\sigma^{\prime}\right)\right|$ and $\left|\mathcal{A}^{(n)}(\sigma)\right\rangle$. This naming convention stems from the simple interpretation of eq. (2.19) in terms of cut diagrams, where one (arbitrarily) associates bra vectors $\langle$.$| with$ the left side of the cut.

\subsection{Subtraction of colour-summed matrix elements}

According to the conventions introduced at the beginning of section 2, the quantities that enter eq. (1.4) are defined as follows:

$$
\begin{aligned}
\lim _{k_{n+1} \rightarrow 0} \mathcal{M}^{(n+1)} & =\mathcal{M}_{\mathrm{SOFT}}^{(n+1)}, \\
\lim _{k_{n+1} \| k_{n}} \mathcal{M}^{(n+1)} & =\mathcal{M}_{\mathrm{COLL}}^{(n+1)} .
\end{aligned}
$$

The counterterm $\mathcal{M}_{\mathrm{SC}}^{(n+1)}$, which is responsible for removing the double counting due to $\mathcal{M}_{\mathrm{SOFT}}^{(n+1)}$ and $\mathcal{M}_{\mathrm{COLL}}^{(n+1)}$ in the soft-collinear region, is by construction:

$$
\mathcal{M}_{\mathrm{SC}}^{(n+1)}=\lim _{k_{n+1} \| k_{n}} \mathcal{M}_{\mathrm{SOFT}}^{(n+1)} \equiv \lim _{k_{n+1} \rightarrow 0} \mathcal{M}_{\mathrm{COLL}}^{(n+1)} .
$$

The last identity in eq. (2.29) can, and will, be used as a check of self-consistency when constructing $\mathcal{M}_{\mathrm{SOFT}}^{(n+1)}$ and $\mathcal{M}_{\mathrm{COLL}}^{(n+1)}$.

The result for the soft limit of real-emission matrix elements can be taken e.g. from ref. [11]:

$$
\mathcal{M}_{\mathrm{SOFT}}^{(n+1)}=\frac{1}{2} g_{S}^{2} \sum_{k, l=1}^{n}[k, l] \mathcal{M}_{k l}^{(n)}
$$

with

$$
\begin{aligned}
{[k, l] } & =\frac{k_{k} \cdot k_{l}}{k_{k} \cdot k_{n+1} k_{l} \cdot k_{n+1}}\left(1-\delta_{k l}\right), \\
\mathcal{M}_{k l}^{(n)} & =-2\left\langle\mathcal{A}^{(n)}\left|\sum_{b} Q^{b}(k) Q^{b}(l)\right| \mathcal{A}^{(n)}\right\rangle .
\end{aligned}
$$


The term in round brackets on the r.h.s. of eq. (2.31) is redundant, since $k_{k}^{2}=0$, but it is useful when one formally manipulates eikonal factors. The operators $Q^{b}(k)$ describe the way in which the colour of gluon $k$ is affected when a soft gluon (which here is always labelled by $n+1$ ) of colour $b$ is emitted by it. In the present context, it is useful to regard the result of $Q^{b}(k)$ as twofold: it changes the colour state of gluon $k$, and it creates gluon $n+1$ with colour $b$. This can be written as follows:

$$
\begin{aligned}
\left\langle a_{k} a_{n+1}\left|Q^{b}(k)\right| c_{k}\right\rangle & =\delta_{b a_{n+1}}\left(Q^{b}(k)\right)_{a_{k} c_{k}}=\delta_{b a_{n+1}}\left(T^{b}\right)_{a_{k} c_{k}} \\
\left(T^{b}\right)_{a c} & =-i f^{b a c} .
\end{aligned}
$$

The definition given here implies that $Q^{b}(l)\left|\mathcal{A}^{(n)}\right\rangle$ is a vector in the colour space of $n+1$ gluons. In the colour subspace of gluon $k$, the operator $Q^{b}(k)$ is hermitian.

The collinear limit of real-emission matrix elements can also be taken from ref. [11]. It reads:

$$
\mathcal{M}_{\mathrm{COLL}}^{(n+1)}=\frac{g_{S}^{2}}{k_{n} \cdot k_{n+1}}\left\{P_{g g}(z) \mathcal{M}^{(n)}+Q_{g g^{\star}}(z) \Re\left(\frac{\left\langle k_{n} k_{n+1}\right\rangle}{\left[k_{n} k_{n+1}\right]} \mathcal{M}_{-+}^{(n)}\right)\right\}
$$

where

$$
\mathcal{M}_{-+}^{(n)}=\left\langle\mathcal{A}_{-}^{(n)} \mid \mathcal{A}_{+}^{(n)}\right\rangle
$$

with $\mathcal{A}_{ \pm}^{(n)}$ the $n$-gluon amplitude at fixed \pm helicity of the $n^{\text {th }}$ gluon (i.e. of the gluon that branches). The momentum fraction $z$ is defined according to:

$$
k_{n}=z\left(k_{n}+k_{n+1}\right) \quad \text { when } k_{n+1} \| k_{n} .
$$

The symbols $P_{g g}(z)$ and $Q_{g g^{\star}}(z)$ denote the Altarelli-Parisi kernel and its azimuthal counterpart, respectively, relevant to $g \rightarrow g g$ branchings. The latter kernel has been introduced in ref. [13], and I refer the reader to that paper for further details. By using the identity

$$
\sum_{b} Q^{b}(n) Q^{b}(n)=C_{A} I
$$

with $I$ being the identity operator in the colour space, I now rewrite eq. (2.35) in a slightly different (but completely equivalent) form, which is better suited to the manipulations I shall carry out in the rest of this paper:

$$
\mathcal{M}_{\mathrm{COLL}}^{(n+1)}=\frac{g_{S}^{2}}{k_{n} \cdot k_{n+1}}\left\{\hat{P}_{g g}(z) \overline{\mathcal{M}}^{(n)}+\hat{Q}_{g g^{\star}}(z) \Re\left(\frac{\left\langle k_{n} k_{n+1}\right\rangle}{\left[k_{n} k_{n+1}\right]} \overline{\mathcal{M}}_{-+}^{(n)}\right)\right\},
$$

where

$$
\begin{aligned}
& \overline{\mathcal{M}}^{(n)}=\left\langle\mathcal{A}^{(n)}\left|\sum_{b} Q^{b}(n) Q^{b}(n)\right| \mathcal{A}^{(n)}\right\rangle, \\
& \overline{\mathcal{M}}_{-+}^{(n)}=\left\langle\mathcal{A}_{-}^{(n)}\left|\sum_{b} Q^{b}(n) Q^{b}(n)\right| \mathcal{A}_{+}^{(n)}\right\rangle,
\end{aligned}
$$

and I have introduced the quantities

$$
\hat{P}_{g g}(z)=\frac{1}{C_{A}} P_{g g}(z), \quad \hat{Q}_{g g^{\star}}(z)=\frac{1}{C_{A}} Q_{g g^{\star}}(z) .
$$


The consistency between eqs. (2.30) and (2.39), in the sense of eq. (2.29), is easy to prove. Let me start from computing the collinear limit of $\mathcal{M}_{\mathrm{SOFT}}^{(n+1)}$. Since the only dependence on $k_{n+1}$ is in the eikonal factors, the relevant quantity is:

$$
\lim _{k_{n+1} \| k_{n}}[k, l]=\frac{1}{1-z} \frac{1}{k_{n} \cdot k_{n+1}}\left(\delta_{k n}+\delta_{l n}\right)\left(1-\delta_{k l}\right),
$$

having used eq. (2.37). Therefore

$$
\lim _{k_{n+1} \| k_{n}} \mathcal{M}_{\mathrm{SOFT}}^{(n+1)}=\frac{g_{S}^{2}}{2} \frac{1}{1-z} \frac{1}{k_{n} \cdot k_{n+1}}\left\{\sum_{l=1}^{n-1} \mathcal{M}_{n l}^{(n)}+\sum_{k=1}^{n-1} \mathcal{M}_{k n}^{(n)}\right\} .
$$

As is proved in appendix B, the colour-conservation condition is

$$
\sum_{k=1}^{n} Q^{b}(k)\left|\mathcal{A}^{(n)}\right\rangle=0 \quad \Longrightarrow \quad \sum_{k=1}^{n-1} Q^{b}(k)\left|\mathcal{A}^{(n)}\right\rangle=-Q^{b}(n)\left|\mathcal{A}^{(n)}\right\rangle
$$

for any colour index $b$. Therefore, by using the definitions of the colour-linked Born's given in eq. (2.32), and the ortho-normality of the colour-vector basis of eq. (2.8), one obtains:

$$
\lim _{k_{n+1} \| k_{n}} \mathcal{M}_{\mathrm{SOFT}}^{(n+1)}=g_{S}^{2} \frac{2}{1-z} \frac{1}{k_{n} \cdot k_{n+1}}\left\langle\mathcal{A}^{(n)}\left|\sum_{b} Q^{b}(n) Q^{b}(n)\right| \mathcal{A}^{(n)}\right\rangle .
$$

This expression is manifestly identical to the soft limit of eq. (2.39), since

$$
\hat{P}_{g g}(z) \stackrel{z \rightarrow 1}{\longrightarrow} \frac{2}{1-z}, \quad \hat{Q}_{g g^{\star}}(z) \stackrel{z \rightarrow 1}{\longrightarrow} 0 .
$$

A comment, which will apply throughout the paper, is necessary here. In general, the reduced kinematic configurations (that enter the Born amplitudes) that one obtains by taking the soft or the collinear limit of a fully-resolved configuration (that enter the realemission amplitudes) need not coincide. However, as explained in ref. [11], in the context of FKS subtraction is possible and convenient to adopt phase-space parametrizations for which the two do coincide. This justifies the fact that I have used the same symbol $\mathcal{A}^{(n)}$ e.g. in eqs. (2.32) and (2.40), which would in general understand different kinematics. I shall use a unique notation for the soft- and collinear-induced Born amplitudes in the rest of the paper. Having said that, I should also like to stress that, even if the two kinematics were different, the results would be unchanged, since in the FKS method soft and collinear singularities are treated separately. The only exception would be in section 6 , where the choice of kinematic configurations in the two limits becomes relevant.

\subsection{Subtraction at fixed colour configurations}

In this section, I shall consider the problem of defining the subtractions of eq. (1.4) for a given colour configuration. This implies that the quantity whose soft and collinear limits one needs to construct is

$$
\mathcal{M}^{(n+1)}\left(a_{1}, \ldots a_{n+1}\right)=\left|\mathcal{A}^{(n+1)}\left(a_{1}, \ldots a_{n+1}\right)\right|^{2} .
$$


The relevant limits can be obtained directly from $\mathcal{M}_{\text {SOFT }}^{(n+1)}$ and $\mathcal{M}_{\text {COLL }}^{(n+1)}$, given in eqs. (2.30) and (2.39) respectively. Formally, one proceeds as follows. One starts from eqs. (2.27) and (2.28), and then fixes the colour configuration $\left\{a_{i}\right\}_{i=1}^{n+1}$ on both sides of those equations by inserting there the projector

$$
\left|a_{1}, \ldots a_{n+1}\right\rangle\left\langle a_{1}, \ldots a_{n+1}\right| .
$$

In the l.h.s.'s, such an insertion indeed results in singling out the colour-dressed amplitude squared of eq. (2.48):

$$
\begin{aligned}
\mathcal{M}^{(n+1)} & =\left\langle\mathcal{A}^{(n+1)} \mid \mathcal{A}^{(n+1)}\right\rangle \\
\longrightarrow\left\langle\mathcal{A}^{(n+1)} \mid a_{1}, \ldots a_{n+1}\right\rangle\left\langle a_{1}, \ldots a_{n+1} \mid \mathcal{A}^{(n+1)}\right\rangle & \equiv \mathcal{M}^{(n+1)}\left(a_{1}, \ldots a_{n+1}\right) .
\end{aligned}
$$

When performing the insertion of the projector of eq. (2.49) in the r.h.s. of eq. (2.27), by using eq. (2.30) one obtains:

$$
\mathcal{M}_{\mathrm{SOFT}}^{(n+1)}\left(a_{1}, \ldots a_{n+1}\right)=\frac{1}{2} g_{S}^{2} \sum_{k, l=1}^{n}[k, l] \mathcal{M}_{k l}^{(n)}\left(a_{1}, \ldots a_{n+1}\right),
$$

where

$$
\mathcal{M}_{k l}^{(n)}\left(a_{1}, \ldots a_{n+1}\right)=-2\left\langle\mathcal{A}^{(n)}\left|Q^{a_{n+1}}(k)\right| a_{1}, \ldots a_{n+1}\right\rangle\left\langle a_{1}, \ldots a_{n+1}\left|Q^{a_{n+1}}(l)\right| \mathcal{A}^{(n)}\right\rangle,
$$

having taken the Kronecker delta of eq. (2.33) into account. Using eqs. (2.12) and (2.33), eq. (2.52) can be easily re-expressed in terms of scalar quantities best suited to numerical computations:

$$
\begin{aligned}
& \mathcal{M}_{k l}^{(n)}\left(a_{1}, \ldots a_{n+1}\right)= \\
& \quad-2 \sum_{b_{k}^{\prime} b_{l}} \mathcal{A}^{(n)}\left(a_{1}, \ldots b_{k}^{\prime} \ldots a_{l} \ldots a_{n}\right)^{\star} \mathcal{Q}_{g g}\left(a_{n+1} ; a_{k}, a_{l}\right)_{b_{k}^{\prime} b_{l}} \mathcal{A}^{(n)}\left(a_{1}, \ldots a_{k} \ldots b_{l} \ldots a_{n}\right)
\end{aligned}
$$

where I have introduced the matrices

$$
\mathcal{Q}_{g g}\left(a_{n+1} ; a_{k}, a_{l}\right)_{b c}=\left(T^{a_{n+1}}\right)_{b a_{k}}\left(T^{a_{n+1}}\right)_{a_{l} c}=f^{a_{n+1} a_{k} b} f^{a_{n+1} a_{l} c} .
$$

As the notation suggests, I stress that the colour index $a_{n+1}$ is not summed over on the r.h.s. of eq. (2.54). By construction, in $\mathrm{SU}(3)$ there are $8^{3} \mathcal{Q}_{g g}$ matrices, of dimension $8 \times 8$.

The computation of the collinear limit is performed along the same lines. One obtains:

$$
\begin{aligned}
\mathcal{M}_{\mathrm{COLL}}^{(n+1)}\left(a_{1}, \ldots a_{n+1}\right)=\frac{g_{S}^{2}}{k_{n} \cdot k_{n+1}} & \left\{\hat{P}_{g g}(z) \overline{\mathcal{M}}^{(n)}\left(a_{1}, \ldots a_{n+1}\right)\right. \\
& \left.+\hat{Q}_{g g^{\star}}(z) \Re\left(\frac{\left\langle k_{n} k_{n+1}\right\rangle}{\left[k_{n} k_{n+1}\right]} \overline{\mathcal{M}}_{-+}^{(n)}\left(a_{1}, \ldots a_{n+1}\right)\right)\right\},
\end{aligned}
$$

with

$$
\begin{aligned}
& \overline{\mathcal{M}}^{(n)}\left(a_{1}, \ldots a_{n+1}\right)=\left\langle\mathcal{A}^{(n)}\left|Q^{a_{n+1}}(n)\right| a_{1}, \ldots a_{n+1}\right\rangle\left\langle a_{1}, \ldots a_{n+1}\left|Q^{a_{n+1}}(n)\right| \mathcal{A}^{(n)}\right\rangle, \\
& \overline{\mathcal{M}}_{-+}^{(n)}\left(a_{1}, \ldots a_{n+1}\right)=\left\langle\mathcal{A}_{-}^{(n)}\left|Q^{a_{n+1}}(n)\right| a_{1}, \ldots a_{n+1}\right\rangle\left\langle a_{1}, \ldots a_{n+1}\left|Q^{a_{n+1}}(n)\right| \mathcal{A}_{+}^{(n)}\right\rangle .
\end{aligned}
$$


These can be re-expressed in terms of scalar quantities, as done for their soft counterparts in eq. (2.53):

$$
\begin{aligned}
& \overline{\mathcal{M}}^{(n)}\left(a_{1}, \ldots a_{n+1}\right)= \\
& \sum_{b_{n}^{\prime} b_{n}} \mathcal{A}^{(n)}\left(a_{1}, \ldots a_{n-1}, b_{n}^{\prime}\right)^{\star} \mathcal{Q}_{g g}\left(a_{n+1} ; a_{n}, a_{n}\right)_{b_{n}^{\prime} b_{n}} \mathcal{A}^{(n)}\left(a_{1}, \ldots a_{n-1}, b_{n}\right), \\
& \overline{\mathcal{M}}_{-+}^{(n)}\left(a_{1}, \ldots a_{n+1}\right)= \\
& \sum_{b_{n}^{\prime} b_{n}} \mathcal{A}_{-}^{(n)}\left(a_{1}, \ldots a_{n-1}, b_{n}^{\prime}\right)^{\star} \mathcal{Q}_{g g}\left(a_{n+1} ; a_{n}, a_{n}\right)_{b_{n}^{\prime} b_{n}} \mathcal{A}_{+}^{(n)}\left(a_{1}, \ldots a_{n-1}, b_{n}\right) .
\end{aligned}
$$

The consistency between eqs. (2.51) and (2.55) in the sense of eq. (2.29) can be proved in the same way as was done at the end of section 2.1 for eqs. (2.30) and (2.39). In fact, apart from eq. (2.43) which holds independently of the treatment of the colours, the key point there is colour conservation as given in eq. (2.45). As is discussed in appendix B, colour conservation is a property that holds at fixed $(n+1)$-gluon colour configurations, which is sufficient to prove the point. Equations (2.54) and (A.4) imply that

$$
\sum_{a_{n+1}} \sum_{a_{n}} \mathcal{Q}_{g g}\left(a_{n+1} ; a_{n}, a_{n}\right)_{b c}=C_{A} \delta_{b c}
$$

which is nothing but the matrix form of eq. (2.38).

\subsection{Subtraction at fixed flows}

I now turn to the definition of subtraction at given flows. The problem is formally more involved than that of section 2.3, because of the lack of the analogue of the projector in eq. (2.49). The basic idea is however still the same. To be definite, I consider the soft limit, the collinear limit being fully analogous. One starts from eq. (2.27), and expresses both sides as sums over $(n+1)$-gluon L- and R-flows:

$$
\begin{aligned}
\lim _{k_{n+1} \rightarrow 0}\left(\sum_{\Sigma, \Sigma^{\prime} \in P_{n+1}^{\prime}} \mathcal{M}^{(n+1)}\left(\Sigma^{\prime}, \Sigma\right)\right) & \equiv \sum_{\Sigma, \Sigma^{\prime} \in P_{n+1}^{\prime}} \lim _{k_{n+1} \rightarrow 0} \mathcal{M}^{(n+1)}\left(\Sigma^{\prime}, \Sigma\right) \\
& =\sum_{\Sigma, \Sigma^{\prime} \in P_{n+1}^{\prime}} \mathcal{M}_{\text {SOFT }}^{(n+1)}\left(\Sigma^{\prime}, \Sigma\right) .
\end{aligned}
$$

At this point, since the representation in terms of flows is unique, the terms with the same $\Sigma$ and $\Sigma^{\prime}$ on the two sides of eq. (2.61) will be the analogues, at fixed flows, of the first two terms of eq. (1.4) - they will play the same roles as eqs. (2.48) and (2.51) played in the case of subtraction at fixed colour configurations.

This procedure is completely trivial as far as the l.h.s. of eq. (2.61) is concerned, since it amounts to using the definition of colour vectors at fixed flows, given in eq. (2.19):

$$
\begin{aligned}
\mathcal{M}^{(n+1)}\left(\Sigma^{\prime}, \Sigma\right) & =\left\langle\mathcal{A}^{(n+1)}\left(\Sigma^{\prime}\right) \mid \mathcal{A}^{(n+1)}(\Sigma)\right\rangle \\
& =\widehat{\mathcal{A}}^{(n+1)}\left(\Sigma^{\prime}\right)^{\star} C\left(\Sigma^{\prime}, \Sigma\right) \widehat{\mathcal{A}}^{(n+1)}(\Sigma),
\end{aligned}
$$


with the colour-flow matrix $C$ defined as in eq. (2.21), with $n \rightarrow n+1$ there. What is less trivial is the r.h.s. of eq. (2.61). The existence of such a decomposition is guaranteed by the fact that the amplitudes relevant to the reduced matrix element $\mathcal{M}_{\text {SOFT }}^{(n+1)}$ live in the $(n+1)$-gluon colour space. However, their colour structures are obtained by means of the operators $Q^{b}$ that act on $n$-gluon colour vectors (see eq. (2.32)), and one therefore obtains the contributions at given $\Sigma$ and $\Sigma^{\prime}$ in a rather indirect way. The easiest way to perform the computation of $\mathcal{M}_{\mathrm{SOFT}}^{(n+1)}\left(\Sigma^{\prime}, \Sigma\right)$ is obviously that of using the decomposition of the $n$-gluon colour vectors in terms of $n$-gluon flows, eq. (2.13), as the starting point. This implies that when computing $\mathcal{M}_{\text {SOFT }}^{(n+1)}$ one will end up dealing with quantities such as:

$$
\sum_{k, l}[k, l]\left\langle\mathcal{A}^{(n)}\left(\sigma^{\prime}\right)\left|\sum_{b} Q^{b}(k) Q^{b}(l)\right| \mathcal{A}^{(n)}(\sigma)\right\rangle .
$$

As is shown in appendix B (see in particular eq. (B.10)), for a given index $l$ and an $n$-gluon R-flow $\sigma$, the vector $Q^{b}(l)\left|\mathcal{A}^{(n)}(\sigma)\right\rangle$ corresponds to the two $(n+1)$-gluon R-flows

$$
I_{+}\left(\sigma^{-1}(l)\right) \sigma, \quad I_{-}\left(\sigma^{-1}(l)\right) \sigma,
$$

with $I_{ \pm}$defined in eqs. (B.7) and (B.8). In the case of L-flows, one finds instead:

$$
I_{+}\left(\sigma^{\prime^{-1}}(k)\right) \sigma^{\prime}, \quad I_{-}\left(\sigma^{\prime-1}(k)\right) \sigma^{\prime} .
$$

This is equivalent to saying that eq. (2.64) defines two maps:

$$
(l, \sigma) \stackrel{I_{+}}{\longrightarrow} \Sigma_{+}, \quad(l, \sigma) \stackrel{I_{-}}{\longrightarrow} \Sigma_{-},
$$

relevant to R-flows. The situation is obviously identical for L-flows:

$$
\left(k, \sigma^{\prime}\right) \stackrel{I_{+}}{\longrightarrow} \Sigma_{+}^{\prime}, \quad\left(k, \sigma^{\prime}\right) \stackrel{I_{-}}{\longrightarrow} \Sigma_{-}^{\prime} .
$$

In order to find the representation that appears on the r.h.s. of eq. (2.61), one has therefore to fix $\Sigma_{ \pm}$and $\Sigma_{ \pm}^{\prime}$, and to invert the maps of eqs. (2.66) and (2.67).

I shall explicitly carry out this procedure in section 2.3.1. Before turning to that, it is worth remarking that by construction the gluon-insertion operators $Q^{b}$ do not affect the dual amplitudes (i.e., they leave the Lorentz structure invariant). Hence, both $Q^{b}\left|\mathcal{A}^{(n)}(\sigma)\right\rangle$ and $\left|\mathcal{A}^{(n)}(\sigma)\right\rangle$ will contain the same n-gluon dual amplitude $\widehat{\mathcal{A}}^{(n)}(\sigma)$. This suggests a procedure alternative to that implied by eq. (2.61). Namely, one may simply fix the $n$ gluon flows that appear in eq. (2.63), and therefore choose not to invert the maps of eqs. (2.66) and (2.67). Rather, these maps will be used to identify all $\mathcal{M}^{(n+1)}\left(\Sigma^{\prime}, \Sigma\right)$ whose sum has a soft limit proportional to the quantity in eq. (2.63). I shall discuss this approach in section 2.3.2.

The physical meaning of the two procedures sketched above is obvious. While in eq. (2.61) one fixes the flows at the level of real-emission matrix elements, in the other case the fixed flows are those of the Born matrix elements. Therefore, I shall refer to these two viewpoints as fixed real flows and fixed Born flows respectively. 


\subsubsection{Fixed real flows}

As was anticipated above, I start by expressing the soft matrix element as a sum over $n$-gluon flows:

$$
\begin{aligned}
\mathcal{M}_{\mathrm{SOFT}}^{(n+1)} & =\sum_{\sigma, \sigma^{\prime} \in P_{n}^{\prime}} \mathcal{M}_{\mathrm{SOFT}}^{(n+1)}\left(\sigma^{\prime}, \sigma\right), \\
\mathcal{M}_{\mathrm{SOFT}}^{(n+1)}\left(\sigma^{\prime}, \sigma\right) & =\frac{1}{2} g_{S}^{2} \sum_{k, l=1}^{n}[k, l] \mathcal{M}_{k l}^{(n)}\left(\sigma^{\prime}, \sigma\right), \\
\mathcal{M}_{k l}^{(n)}\left(\sigma^{\prime}, \sigma\right) & =-2\left\langle\mathcal{A}^{(n)}\left(\sigma^{\prime}\right)\left|\sum_{b} Q^{b}(k) Q^{b}(l)\right| \mathcal{A}^{(n)}(\sigma)\right\rangle .
\end{aligned}
$$

In order to compute explicitly the colour-linked Born's that appear in eq. (2.70), I introduce the following $(n+1)$-gluon colour vectors associated with an underlying $n$-body dynamics:

$$
\left|\mathcal{A}_{\mathrm{EXT}}^{(n)}(\Sigma)\right\rangle=\sum_{\left\{a_{i}\right\}_{i=1}^{n+1}} \Lambda\left(\left\{a_{i}\right\}, \Sigma\right) \widehat{\mathcal{A}}^{(n)}\left(\Sigma_{n+\mathrm{Y}}\right)\left|a_{1}, \ldots a_{n+1}\right\rangle,
$$

where $\Sigma \in P_{n+1}^{\prime}$, and I defined

$$
\Sigma_{n+1}=(\Sigma(1), \ldots n+1, \ldots \Sigma(n+1)) .
$$

By using the results of appendix B, and as already anticipated in eq. (2.64), one obtains:

$$
\sum_{b} Q^{b}(l)\left|\mathcal{A}^{(n)}(\sigma)\right\rangle=\left|\mathcal{A}_{\mathrm{EXT}}^{(n)}\left(I_{+}\left(\sigma^{-1}(l)\right) \sigma\right)\right\rangle-\left|\mathcal{A}_{\mathrm{EXT}}^{(n)}\left(I_{-}\left(\sigma^{-1}(l)\right) \sigma\right)\right\rangle .
$$

I can now use eq. (2.73) in eq. (2.70) since, for any two $n$-gluon colour vectors $\left\langle v^{\prime}\right|$ and $|v\rangle$, the follow identity holds:

$$
\sum_{b}\left\langle v^{\prime}\left|Q^{b}(k) Q^{b}(l)\right| v\right\rangle=\sum_{b, c} \delta_{b c}\left\langle v^{\prime}\left|Q^{b}(k) Q^{c}(l)\right| v\right\rangle=\sum_{b, c} \delta_{b c}\left\langle w^{\prime} b \mid w c\right\rangle=\sum_{b, c}\left\langle w^{\prime} b \mid w c\right\rangle,
$$

since $\delta_{b c}$ is automatically enforced by the dot product of the colour vectors, which receives the contribution $\langle b \mid c\rangle=\delta_{b c}$ from the $(n+1)^{\text {th }}$-gluon subspace. Then:

$$
\begin{aligned}
\mathcal{M}_{k l}^{(n)}\left(\sigma^{\prime}, \sigma\right)=-2 & \left\{\left\langle\mathcal{A}_{\mathrm{EXT}}^{(n)}\left(I_{+}\left(\sigma^{\prime^{-1}}(k)\right) \sigma^{\prime}\right)\right|-\left\langle\mathcal{A}_{\mathrm{EXT}}^{(n)}\left(I_{-}\left(\sigma^{\prime^{-1}}(k)\right) \sigma^{\prime}\right)\right|\right\} \\
& \left\{\left|\mathcal{A}_{\mathrm{EXT}}^{(n)}\left(I_{+}\left(\sigma^{-1}(l)\right) \sigma\right)\right\rangle-\left|\mathcal{A}_{\mathrm{EXT}}^{(n)}\left(I_{-}\left(\sigma^{-1}(l)\right) \sigma\right)\right\rangle\right\} .
\end{aligned}
$$

One can now change the labellings in the sums that appear on the r.h.s. of eq. (2.69), $\sum_{k l} f(k, l)=\sum_{i j} f\left(\sigma^{\prime}(i), \sigma(j)\right)$. Furthermore, one exploits eq. (B.13), which can also be conveniently extended:

$$
I_{-}(1) \sigma=I_{+}(0) \sigma, \quad\left|\mathcal{A}_{\mathrm{EXT}}^{(n)}\left(I_{+}(0) \sigma\right)\right\rangle \equiv\left|\mathcal{A}_{\mathrm{EXT}}^{(n)}\left(I_{+}(n) \sigma\right)\right\rangle,
$$

where the last condition can be imposed because of the cyclicity of the trace. One then obtains:

$$
\begin{aligned}
\mathcal{M}_{\mathrm{SOFT}}^{(n+1)} & \left(\sigma^{\prime}, \sigma\right)=-g_{S}^{2} \sum_{i, j=1}^{n}\left[\sigma^{\prime}(i), \sigma(j)\right]\left\{\left\langle\mathcal{A}_{\mathrm{EXT}}^{(n)}\left(I_{+}(i) \sigma^{\prime}\right) \mid \mathcal{A}_{\mathrm{EXT}}^{(n)}\left(I_{+}(j) \sigma\right)\right\rangle\right. \\
& -\left\langle\mathcal{A}_{\mathrm{EXT}}^{(n)}\left(I_{+}(i) \sigma^{\prime}\right) \mid \mathcal{A}_{\mathrm{EXT}}^{(n)}\left(I_{+}(j-1) \sigma\right)\right\rangle-\left\langle\mathcal{A}_{\mathrm{EXT}}^{(n)}\left(I_{+}(i-1) \sigma^{\prime}\right) \mid \mathcal{A}_{\mathrm{EXT}}^{(n)}\left(I_{+}(j) \sigma\right)\right\rangle \\
& \left.+\left\langle\mathcal{A}_{\mathrm{EXT}}^{(n)}\left(I_{+}(i-1) \sigma^{\prime}\right) \mid \mathcal{A}_{\mathrm{EXT}}^{(n)}\left(I_{+}(j-1) \sigma\right)\right\rangle\right\} .
\end{aligned}
$$


By relabelling $j-1 \rightarrow j$ in the second and fourth terms on the r.h.s. of eq. (2.77), and $i-1 \rightarrow i$ in the third and fourth terms, and by using the second equality in eq. (2.76), one finally gets:

$$
\begin{aligned}
\mathcal{M}_{\mathrm{SOFT}}^{(n+1)}\left(\sigma^{\prime}, \sigma\right)=-g_{S}^{2} \sum_{i, j=1}^{n} & \left\{\left[\sigma^{\prime}(i), \sigma(j)\right]-\left[\sigma^{\prime}(i), \sigma(j+1)\right]\right. \\
& \left.-\left[\sigma^{\prime}(i+1), \sigma(j)\right]+\left[\sigma^{\prime}(i+1), \sigma(j+1)\right]\right\} \\
& \times\left\langle\mathcal{A}_{\mathrm{EXT}}^{(n)}\left(I_{+}(i) \sigma^{\prime}\right) \mid \mathcal{A}_{\mathrm{EXT}}^{(n)}\left(I_{+}(j) \sigma\right)\right\rangle,
\end{aligned}
$$

where, as a consequence of the relabellings mentioned above, one must understand

$$
\sigma(n+1)=\sigma(1) .
$$

At variance with eq. (2.70), eq. (2.78) contains a single $(n+1)$-gluon closed flow for a given $(i, j)$ pair. This is what renders it easy to cast the sum over $\sigma$ and $\sigma^{\prime}$ (see eq. (2.68)) of the quantities in eq. (2.78) in the same form as the r.h.s. of eq. (2.61). One can start by using the following identity, which I write for R-flows to be definite (the case of L-flows being identical):

$$
\sum_{\sigma \in P_{n}^{\prime}} \sum_{j=1}^{n} f(\sigma, j)=\sum_{\sigma \in P_{n}^{\prime}} \sum_{j=1}^{n} \sum_{\Sigma \in P_{n+1}^{\prime}} \delta\left(\Sigma, I_{+}(j) \sigma\right) f(\sigma, j),
$$

with $\delta$ on the r.h.s. of eq. (2.80) the Kronecker delta. The identity above holds true since

$$
1=\sum_{\Sigma \in P_{n+1}^{\prime}} \delta\left(\Sigma, I_{+}(j) \sigma\right) \quad \text { for given } \sigma, j
$$

which is a consequence of the fact that, at fixed $\sigma$ and $j$, the $(n+1)$-gluon flow $I_{+}(j) \sigma$ always exists and is unique. Now, the sum over $\Sigma$ in eq. (2.80) will play the same role as that on the r.h.s. of eq. (2.61). Hence, one can exploit the $\delta$ in eq. (2.80) to get rid of the sums over $\sigma$ and $j$. This implies solving the equation:

$$
\begin{aligned}
I_{+}(j) \sigma=\Sigma \Longleftrightarrow & \\
& (\sigma(1), \ldots \sigma(j), n+1, \sigma(j+1), \ldots \sigma(n)) \\
= & (\Sigma(1), \ldots \Sigma(j), \Sigma(j+1), \Sigma(j+2), \ldots \Sigma(n+1))
\end{aligned}
$$

for a given $\Sigma$. It is immediate to see that this solution always exists:

$$
\begin{aligned}
\sigma & =\Sigma_{n+1}, \\
j & =\Sigma^{-1}(n+1)-1,
\end{aligned}
$$

and is unique. In other words, there is one-to-one correspondence between pairs composed of one $P_{n}^{\prime}$ permutation and one integer, and $P_{n+1}^{\prime}$ permutations. Equation (2.80) then becomes:

$$
\sum_{\sigma \in P_{n}^{\prime}} \sum_{j=1}^{n} f(\sigma, j)=\sum_{\Sigma \in P_{n+1}^{\prime}} f\left(\Sigma_{n+1}, \Sigma^{-1}(n+1)-1\right) .
$$


Equation (2.86) is consistent with simple counting: the $n ! P_{n+1}^{\prime}$ permutations can be obtained by inserting the integer $n+1$ in all the $n$ possible ways (not $n+1$, because of non-cyclicity) in each of the $(n-1) ! P_{n}^{\prime}$ permutations: $n !=n \times(n-1) !$.

These arguments, when applied to L-flows, give

$$
\begin{aligned}
\sigma^{\prime} & =\Sigma_{n+1}^{\prime}, \\
i & =\Sigma^{\prime-1}(n+1)-1 .
\end{aligned}
$$

The results above can now be used in eqs. (2.68) and (2.78). One obtains:

$$
\begin{aligned}
& \mathcal{M}_{\mathrm{SOFT}}^{(n+1)}= \sum_{\Sigma, \Sigma^{\prime} \in P_{n+1}^{\prime}} \mathcal{M}_{\mathrm{SOFT}}^{(n+1)}\left(\Sigma^{\prime}, \Sigma\right) \\
& \mathcal{M}_{\mathrm{SOFT}}^{(n+1)}\left(\Sigma^{\prime}, \Sigma\right)=-g_{S}^{2}\left\{\left[\Sigma^{\prime}\left(\Sigma^{\prime-1}(n+1)-1\right), \Sigma\left(\Sigma^{-1}(n+1)-1\right)\right]\right. \\
&-\left[\Sigma^{\prime}\left(\Sigma^{\prime-1}(n+1)-1\right), \Sigma\left(\Sigma^{-1}(n+1)+1\right)\right] \\
& \quad-\left[\Sigma^{\prime}\left(\Sigma^{\prime-1}(n+1)+1\right), \Sigma\left(\Sigma^{-1}(n+1)-1\right)\right] \\
&\left.\quad+\left[\Sigma^{\prime}\left(\Sigma^{\prime-1}(n+1)+1\right), \Sigma\left(\Sigma^{-1}(n+1)+1\right)\right]\right\} \mathcal{M}_{\mathrm{EXT}}^{(n)}\left(\Sigma^{\prime}, \Sigma\right), \\
& \mathcal{M}_{\mathrm{EXT}}^{(n)}\left(\Sigma^{\prime}, \Sigma\right)=\left\langle\mathcal{A}_{\mathrm{EXT}}^{(n)}\left(\Sigma^{\prime}\right) \mid \mathcal{A}_{\mathrm{EXT}}^{(n)}(\Sigma)\right\rangle .
\end{aligned}
$$

In the evaluation of the eikonal factors that appear in eq. (2.90), one must take the analogues of eqs. (2.6) and (2.79) into account, namely

$$
\Sigma(1)=1, \quad \Sigma(n+2)=\Sigma(1),
$$

and similarly for $\Sigma^{\prime}$. Note that the second identity in eq. (2.92) is enforced by the solution of eq. (2.83) in the case when $n+1$ occupies the rightmost position in $I_{+}(j) \sigma$. Equations (2.90) and (2.91) only depend on $\Sigma$ and $\Sigma^{\prime}$, and are therefore in the form suited to be used in eq. (2.61). In terms of scalar quantities, eq. (2.91) reads as follows:

$$
\mathcal{M}_{\mathrm{EXT}}^{(n)}\left(\Sigma^{\prime}, \Sigma\right)=\widehat{\mathcal{A}}^{(n)}\left(\Sigma_{n+1}^{\prime}\right)^{\star} C\left(\Sigma^{\prime}, \Sigma\right) \widehat{\mathcal{A}}^{(n)}\left(\Sigma_{n+1}\right) .
$$

I now address the case of the collinear limit, which I deal with as was done for the soft limit. Namely, in eqs. (2.39)-(2.41) I use the representation of the amplitude in terms of flows, eq. (2.13), and its analogue for L-flows:

$$
\left\langle\mathcal{A}^{(n)}\right|=\sum_{\sigma^{\prime} \in P_{n}^{\prime}}\left\langle\mathcal{A}^{(n)}\left(\sigma^{\prime}\right)\right| .
$$

In doing that, I explicitly express the real part that appears in the term proportional to $Q_{g g^{\star}}$ in eq. (2.39), by writing it as the sum of its argument plus the complex conjugate of the latter; this is necessary in order to identify $\sigma$ with the R-flow, and $\sigma^{\prime}$ with the L-flow. I thus obtain:

$$
\mathcal{M}_{\mathrm{COLL}}^{(n+1)}=\sum_{\sigma, \sigma^{\prime} \in P_{n}^{\prime}} \mathcal{M}_{\mathrm{COLL}}^{(n+1)}\left(\sigma^{\prime}, \sigma\right)
$$


where

$$
\begin{aligned}
\mathcal{M}_{\mathrm{COLL}}^{(n+1)}\left(\sigma^{\prime}, \sigma\right)=\frac{g_{S}^{2}}{k_{n} \cdot k_{n+1}} & \left\{\hat{P}_{g g}(z) \overline{\mathcal{M}}^{(n)}\left(\sigma^{\prime}, \sigma\right)\right. \\
& \left.+\frac{1}{2} \hat{Q}_{g g^{\star}}(z)\left(\frac{\left\langle k_{n} k_{n+1}\right\rangle}{\left[k_{n} k_{n+1}\right]} \overline{\mathcal{M}}_{-+}^{(n)}\left(\sigma^{\prime}, \sigma\right)+\frac{\left[k_{n} k_{n+1}\right]}{\left\langle k_{n} k_{n+1}\right\rangle} \overline{\mathcal{M}}_{+-}^{(n)}\left(\sigma^{\prime}, \sigma\right)\right)\right\},
\end{aligned}
$$

and

$$
\begin{aligned}
& \overline{\mathcal{M}}^{(n)}\left(\sigma^{\prime}, \sigma\right)=\left\langle\mathcal{A}^{(n)}\left(\sigma^{\prime}\right)\left|\sum_{b} Q^{b}(n) Q^{b}(n)\right| \mathcal{A}^{(n)}(\sigma)\right\rangle, \\
& \overline{\mathcal{M}}_{\lambda \bar{\lambda}}^{(n)}\left(\sigma^{\prime}, \sigma\right)=\left\langle\mathcal{A}_{\lambda}^{(n)}\left(\sigma^{\prime}\right)\left|\sum_{b} Q^{b}(n) Q^{b}(n)\right| \mathcal{A}_{\bar{\lambda}}^{(n)}(\sigma)\right\rangle .
\end{aligned}
$$

Given that eq. (2.98) is essentially identical to eq. (2.97), I shall deal only with $\overline{\mathcal{M}}^{(n)}$ in the following; the term proportional to the azimuthal kernel $\hat{Q}_{g g^{\star}}(z)$ will be reinstated at the end. By comparing eq. (2.97) with eq. (2.70) one gets:

$$
\overline{\mathcal{M}}^{(n)}\left(\sigma^{\prime}, \sigma\right)=-\frac{1}{2} \mathcal{M}_{n n}^{(n)}\left(\sigma^{\prime}, \sigma\right) .
$$

Hence, from eq. (2.75) one obtains:

$$
\begin{aligned}
\overline{\mathcal{M}}^{(n)}\left(\sigma^{\prime}, \sigma\right)= & \left\langle\mathcal{A}_{\mathrm{EXT}}^{(n)}\left(I_{+}\left(\sigma^{\prime^{-1}}(n)\right) \sigma^{\prime}\right) \mid \mathcal{A}_{\mathrm{EXT}}^{(n)}\left(I_{+}\left(\sigma^{-1}(n)\right) \sigma\right)\right\rangle \\
& -\left\langle\mathcal{A}_{\mathrm{EXT}}^{(n)}\left(I_{+}\left(\sigma^{\prime-1}(n)\right) \sigma^{\prime}\right) \mid \mathcal{A}_{\mathrm{EXT}}^{(n)}\left(I_{-}\left(\sigma^{-1}(n)\right) \sigma\right)\right\rangle \\
& -\left\langle\mathcal{A}_{\mathrm{EXT}}^{(n)}\left(I_{-}\left(\sigma^{\prime-1}(n)\right) \sigma^{\prime}\right) \mid \mathcal{A}_{\mathrm{EXT}}^{(n)}\left(I_{+}\left(\sigma^{-1}(n)\right) \sigma\right)\right\rangle \\
& +\left\langle\mathcal{A}_{\mathrm{EXT}}^{(n)}\left(I_{-}\left(\sigma^{\prime-1}(n)\right) \sigma^{\prime}\right) \mid \mathcal{A}_{\mathrm{EXT}}^{(n)}\left(I_{-}\left(\sigma^{-1}(n)\right) \sigma\right)\right\rangle .
\end{aligned}
$$

The $(n+1)$-gluon L- and R-flows that appear on the r.h.s. of eq. (2.100) are such that:

$$
\begin{array}{llll}
I_{+}\left(\sigma^{\prime-1}(n)\right) \sigma^{\prime}, & I_{+}\left(\sigma^{-1}(n)\right) \sigma & \longrightarrow & (\ldots n, n+1, \ldots), \\
I_{-}\left(\sigma^{\prime-1}(n)\right) \sigma^{\prime}, & I_{-}\left(\sigma^{-1}(n)\right) \sigma & \longrightarrow & (\ldots n+1, n, \ldots),
\end{array}
$$

that is, $n$ and $n+1$ must be contiguous. This implies that all flows for which this condition is not satisfied correspond to matrix elements that do not diverge in the collinear limit. In order to proceed as was done in the case of the soft limit, I can now impose the analogues of eq. (2.82). By considering only R-flows to be definite, there are two possible cases as shown in eqs. (2.101) and (2.102), and therefore one has either

$$
I_{+}\left(\sigma^{-1}(n)\right) \sigma \equiv \Sigma=(\ldots n, n+1, \ldots)
$$

or

$$
I_{-}\left(\sigma^{-1}(n)\right) \sigma \equiv \Sigma=(\ldots n+1, n, \ldots) .
$$

These equations impose the following constraints on $\Sigma$ :

$$
\Sigma^{-1}(n)=\Sigma^{-1}(n+1)-1
$$


in the case of eq. (2.103), and

$$
\Sigma^{-1}(n)=\Sigma^{-1}(n+1)+1
$$

in the case of eq. (2.104). The conditions of eqs. (2.105) and (2.106) are obviously mutually exclusive. By repeating the same exercise for L-flows, and by using eq. (2.100), one finally arrives at:

$$
\sum_{\sigma, \sigma^{\prime} \in P_{n}^{\prime}} \overline{\mathcal{M}}^{(n)}\left(\sigma^{\prime}, \sigma\right)=\sum_{\Sigma, \Sigma^{\prime} \in P_{n+1}^{\prime}} \delta\left(\Sigma^{\prime}, \Sigma\right) \mathcal{M}_{\mathrm{EXT}}^{(n)}\left(\Sigma^{\prime}, \Sigma\right)
$$

where $\mathcal{M}_{\mathrm{EXT}}^{(n)}$ is given in eq. (2.93), and

$$
\delta\left(\Sigma^{\prime}, \Sigma\right)=\sum_{\alpha=-1,1} \sum_{\beta=-1,1} \alpha \beta \delta\left(\Sigma^{\prime^{-1}}(n), \Sigma^{\prime^{-1}}(n+1)+\alpha\right) \delta\left(\Sigma^{-1}(n), \Sigma^{-1}(n+1)+\beta\right) .
$$

The $\delta$ symbols that appear on the r.h.s. of eq. (2.108) are the ordinary Kronecker delta's. Note that only one of the four terms in the sum in eq. (2.108) can be different from zero at given $\Sigma$ and $\Sigma^{\prime}$. These four terms corresponds to those on the r.h.s. of eq. (2.100); their signs there are equivalent to the factor $\alpha \beta$ in eq. (2.108). Furthermore, $\delta\left(\Sigma^{\prime}, \Sigma\right)$ is equal to zero when $n$ is not contiguous to $n+1$ in either the L- or R-flow. These properties of eq. (2.108) ensure that eq. (2.107) holds. Putting all together, one obtains:

$$
\begin{aligned}
\mathcal{M}_{\mathrm{COLL}}^{(n+1)}= & \sum_{\Sigma, \Sigma^{\prime} \in P_{n+1}^{\prime}} \mathcal{M}_{\mathrm{COLL}}^{(n+1)}\left(\Sigma^{\prime}, \Sigma\right) \\
\mathcal{M}_{\mathrm{COLL}}^{(n+1)}\left(\Sigma^{\prime}, \Sigma\right)= & \frac{g_{S}^{2}}{k_{n} \cdot k_{n+1}} \delta\left(\Sigma^{\prime}, \Sigma\right)\left\{\hat{P}_{g g}(z) \mathcal{M}_{\mathrm{EXT}}^{(n)}\left(\Sigma^{\prime}, \Sigma\right)\right. \\
& \left.+\frac{1}{2} \hat{Q}_{g g^{\star}}(z)\left(\frac{\left\langle k_{n} k_{n+1}\right\rangle}{\left[k_{n} k_{n+1}\right]} \mathcal{M}_{\mathrm{EXT}-+}^{(n)}\left(\Sigma^{\prime}, \Sigma\right)+\frac{\left[k_{n} k_{n+1}\right]}{\left\langle k_{n} k_{n+1}\right\rangle} \mathcal{M}_{\mathrm{EXT}+-}^{(n)}\left(\Sigma^{\prime}, \Sigma\right)\right)\right\}
\end{aligned}
$$

where

$$
\mathcal{M}_{\mathrm{EXT} \lambda \bar{\lambda}}^{(n)}\left(\Sigma^{\prime}, \Sigma\right)=\left\langle\mathcal{A}_{\mathrm{EXT} \lambda}^{(n)}\left(\Sigma^{\prime}\right) \mid \mathcal{A}_{\mathrm{EXT} \bar{\lambda}}^{(n)}(\Sigma)\right\rangle,
$$

or, in terms of scalar quantities:

$$
\mathcal{M}_{\mathrm{EXT \lambda \overline { \lambda }}}^{(n)}\left(\Sigma^{\prime}, \Sigma\right)=\widehat{\mathcal{A}}_{\lambda}^{(n)}\left(\Sigma_{n+\mp}^{\prime}\right)^{\star} C\left(\Sigma^{\prime}, \Sigma\right) \widehat{\mathcal{A}}_{\bar{\lambda}}^{(n)}\left(\Sigma_{n+千}\right) .
$$

The consistency between eqs. (2.90) and (2.110) in the sense of eq. (2.29) can be proved by direct computation, using eq. (2.43). One can verify that the Kronecker delta's that appear on the r.h.s. of the latter equation combine effectively to give $\delta\left(\Sigma^{\prime}, \Sigma\right)$ defined in eq. (2.108); the quickest way to see this is that of observing that the linear combination of the four eikonals in eq. (2.90) can be rewritten as follows:

$$
\sum_{\alpha=-1,1} \sum_{\beta=-1,1} \alpha \beta\left[\Sigma^{\prime}\left(\Sigma^{\prime-1}(n+1)+\alpha\right), \Sigma\left(\Sigma^{-1}(n+1)+\beta\right)\right],
$$

i.e. a similar form as eq. (2.108). It is interesting to notice that the collinear limit of an eikonal, eq. (2.43), constrains $n$ to be contiguous to $n+1$ in either the R- or the L-flow, but not necessarily in both. However, when $n$ and $n+1$ are contiguous only in one of the two 
flows, the linear combination in eq. (2.113) contains two divergent eikonals with opposite signs, and therefore is ultimately non-singular. This is the reason why eq. (2.108) forces $n$ and $n+1$ to be contiguous on both sides of the cut.

I point out that the treatment of the collinear limit as done above is quite similar to that of the soft limit owing to the use of eq. (2.39) rather than of eq. (2.35) as a starting point, and in particular to the presence of the $Q^{b}$ operators in the reduced $n$-gluon matrix elements that appear in the former expression. The same technical trick will be adopted when treating the case of quark-gluon amplitudes.

\subsubsection{Fixed Born flows}

I now discuss the second of the strategies outlined at the beginning of section 2.3. I shall make extensive use of the results obtained in section 2.3.1, and I shall start from considering the soft limit.

Fixing Born flows is equivalent to the following interpretation of eq. (2.80): at a given $\sigma$, one considers all $(n+1)$-gluon flows $\Sigma=I_{+}(j) \sigma$ that arise when performing the sum over $j$. Equation (2.78) then implies that such $\Sigma$ 's will constitute the set:

$$
\zeta(\sigma)=\bigcup_{l=1}^{n}\left\{I_{+}\left(\sigma^{-1}(l)\right) \sigma\right\} \equiv \bigcup_{j=1}^{n}\left\{I_{+}(j) \sigma\right\} .
$$

The discussion that follows eq. (2.80) means that:

$$
\begin{aligned}
\zeta\left(\sigma_{1}\right) \bigcap \zeta\left(\sigma_{2}\right) & =\emptyset \quad \text { if } \sigma_{1} \neq \sigma_{2}, \\
\bigcup_{\sigma \in P_{n}^{\prime}} \zeta(\sigma) & =P_{n+1}^{\prime} .
\end{aligned}
$$

Therefore, the sets $\zeta(\sigma)$ achieve a non-overlapping partition of the space of the $(n+1)$-gluon flows into subsets associated with $n$-gluon flows. One is thus led to define $(n+1)$-gluon matrix elements at fixed $n$-gluon flows:

$$
\begin{aligned}
\mathcal{M}^{(n+1)}\left(\sigma^{\prime}, \sigma\right) & =\sum_{\Sigma \in \zeta(\sigma)} \sum_{\Sigma^{\prime} \in \zeta\left(\sigma^{\prime}\right)} \mathcal{M}^{(n+1)}\left(\Sigma^{\prime}, \Sigma\right), \\
\mathcal{M}^{(n+1)} & =\sum_{\sigma, \sigma^{\prime} \in P_{n}^{\prime}} \mathcal{M}^{(n+1)}\left(\sigma^{\prime}, \sigma\right),
\end{aligned}
$$

where eq. (2.118) follows from eqs. (2.115) and (2.116). The derivation carried out in section 2.3.1 then implies that the soft limit of $\mathcal{M}^{(n+1)}\left(\sigma^{\prime}, \sigma\right)$ is equal to $\mathcal{M}_{\text {SOFT }}^{(n+1)}\left(\sigma^{\prime}, \sigma\right)$ defined in eq. (2.69). The colour-linked Born's of eq. (2.70) read, in terms of scalar quantities:

$$
\begin{aligned}
\mathcal{M}_{k l}^{(n)}\left(\sigma^{\prime}, \sigma\right)=-2 \widehat{\mathcal{A}}^{(n)}\left(\sigma^{\prime}\right)^{\star} & {\left[C\left(I_{+}\left(\sigma^{\prime^{-1}}(k)\right) \sigma^{\prime}, I_{+}\left(\sigma^{-1}(l)\right) \sigma\right)\right.} \\
& -C\left(I_{+}\left(\sigma^{\prime^{-1}}(k)\right) \sigma^{\prime}, I_{-}\left(\sigma^{-1}(l)\right) \sigma\right) \\
& -C\left(I_{-}\left(\sigma^{\prime^{-1}}(k)\right) \sigma^{\prime}, I_{+}\left(\sigma^{-1}(l)\right) \sigma\right) \\
& \left.+C\left(I_{-}\left(\sigma^{\prime^{-1}}(k)\right) \sigma^{\prime}, I_{-}\left(\sigma^{-1}(l)\right) \sigma\right)\right] \widehat{\mathcal{A}}^{(n)}(\sigma) .
\end{aligned}
$$


The case of the collinear limit is analogous to that of the soft one. The relevant matrix element, $\mathcal{M}_{\mathrm{COLL}}^{(n+1)}\left(\sigma^{\prime}, \sigma\right)$, has already been defined, see eq. (2.96). Since the $n$-gluon flows are kept fixed here, the explicit expansion performed in eq. (2.100) is not needed, and one can exploit eq. (2.38) to rewrite:

$$
\begin{aligned}
\mathcal{M}_{\mathrm{COLL}}^{(n+1)}\left(\sigma^{\prime}, \sigma\right)=\frac{g_{S}^{2}}{k_{n} \cdot k_{n+1}} & \left\{P_{g g}(z) \mathcal{M}^{(n)}\left(\sigma^{\prime}, \sigma\right)\right. \\
+ & \left.\frac{1}{2} Q_{g g^{\star}}(z)\left(\frac{\left\langle k_{n} k_{n+1}\right\rangle}{\left[k_{n} k_{n+1}\right]} \mathcal{M}_{-+}^{(n)}\left(\sigma^{\prime}, \sigma\right)+\frac{\left[k_{n} k_{n+1}\right]}{\left\langle k_{n} k_{n+1}\right\rangle} \mathcal{M}_{+-}^{(n)}\left(\sigma^{\prime}, \sigma\right)\right)\right\} .
\end{aligned}
$$

In terms of scalar quantities, $\mathcal{M}^{(n)}\left(\sigma^{\prime}, \sigma\right)$ is given in eq. (2.20), and

$$
\mathcal{M}_{\lambda \bar{\lambda}}^{(n)}\left(\sigma^{\prime}, \sigma\right)=\widehat{\mathcal{A}}_{\lambda}^{(n)}\left(\sigma^{\prime}\right)^{\star} C\left(\sigma^{\prime}, \sigma\right) \widehat{\mathcal{A}}_{\bar{\lambda}}^{(n)}(\sigma) .
$$

On the other hand, the expansion of eq. (2.100) serves to define the analogue of the set $\zeta(\sigma)$, namely:

$$
\zeta_{\mathrm{C}}(\sigma)=\left\{I_{+}\left(\sigma^{-1}(n)\right) \sigma, I_{-}\left(\sigma^{-1}(n)\right) \sigma\right\}
$$

At this point, the quantity whose collinear limit is given by eq. (2.120) can be defined in the same way as that in eq. (2.117), namely:

$$
\sum_{\Sigma \in \zeta_{\mathrm{C}}(\sigma)} \sum_{\Sigma^{\prime} \in \zeta_{\mathrm{C}}\left(\sigma^{\prime}\right)} \mathcal{M}^{(n+1)}\left(\Sigma^{\prime}, \Sigma\right)
$$

However, this is not particularly convenient, since the soft limit of eq. (2.123) is not eq. (2.69), which renders it impossible to properly define the subtractions of eq. (1.4). On the other hand, one observes that

$$
\zeta_{\mathrm{C}}(\sigma) \subseteq \zeta(\sigma),
$$

and that all flows belonging to the (generally non empty) set

$$
\zeta(\sigma) \backslash \zeta_{\mathrm{C}}(\sigma)
$$

correspond to matrix elements which are non-singular in the collinear limit. These two facts imply that not only the quantity in eq. (2.123), but also $\mathcal{M}^{(n+1)}\left(\sigma^{\prime}, \sigma\right)$ defined in eq. (2.117) has the collinear limit given by eq. (2.120), or by its fully equivalent form eq. (2.96). One can then conclude that, at fixed $\sigma$ and $\sigma^{\prime}$, the quantities $\mathcal{M}^{(n+1)}\left(\sigma^{\prime}, \sigma\right)$, $\mathcal{M}_{\mathrm{SOFT}}^{(n+1)}\left(\sigma^{\prime}, \sigma\right)$, and $\mathcal{M}_{\mathrm{COLL}}^{(n+1)}\left(\sigma^{\prime}, \sigma\right)$ have all the properties required to play the same roles as the first three matrix elements in eq. (1.4). As far as the soft-collinear counterterm is concerned, it is well defined thanks to the consistency of eqs. (2.69) and (2.96) in the sense of eq. (2.29). This can be proved exactly as done in section 2.1, thanks to eq. (B.14), i.e. to colour conservation at fixed Born flows.

\section{Quark-gluon amplitudes}

I now turn to discussing the case of amplitudes where both quarks and gluons are present. The real-emission process will contain $q$ quark-antiquark pairs, and $n+1$ gluons:

$$
0 \longrightarrow 2 q+(n+1) \text {. }
$$


The $i^{\text {th }}$ parton entering this process will be labelled according to the following conventions: ${ }^{3}$

$$
\begin{aligned}
-2 q \leq i \leq-q-1 & \longrightarrow \text { antiquarks } \\
-q \leq i \leq-1 & \longrightarrow \text { quarks } \\
1 \leq i \leq n+1 & \longrightarrow \text { gluons. }
\end{aligned}
$$

An equal-flavour $q \bar{q}$ pair will correspond to indices $i=-p$ and $i=-p-q$; for the moment, I shall limit myself to discussing the case of one pair per quark flavour. The quarks may be massless of massive; there is no need to distinguish these two cases, since the FKS subtraction formulae do not explicitly depend on quark masses (with the obvious exception that a massive $q \bar{q}$ pair cannot induce a collinear singularity — in the following, I shall always understand that a quark or an antiquark is massless when is involved in a collinear branching). The structure of the underlying Born amplitudes will depend on the type of limit considered. When one of the gluons becomes soft, or two gluons become collinear, or one quark/antiquark becomes collinear to a gluon, the Born process is as follows:

$$
0 \longrightarrow 2 q+n
$$

In other words, this is the situation in which the FKS parton is a gluon; consistently with what was done in section 2, such a gluon will have label $n+1$. Its FKS sisters will be labelled by $n,-q$, and $-2 q$ for $g \rightarrow g g, q \rightarrow q g$, and $\bar{q} \rightarrow \bar{q} g$ branchings respectively. At the Born level, gluon number $n+1$ in eq. (3.4) will not appear. The analogues of the labelling of eqs. (3.2)-(3.4) will thus be:

$$
\begin{aligned}
-2 q \leq i \leq-q-1 & \longrightarrow \text { antiquarks } \\
-q \leq i \leq-1 & \longrightarrow \text { quarks } \\
1 \leq i \leq n & \longrightarrow \text { gluons. }
\end{aligned}
$$

On the other hand, in the case when the FKS parton is a quark, i.e. for a $g \rightarrow q \bar{q}$ branching, the underlying Born will be a process:

$$
0 \longrightarrow 2(q-1)+(n+2)
$$

The analogues of the labelling of eqs. (3.2)-(3.4) will thus be:

$$
\begin{aligned}
& -2 q+1 \leq i \leq-q-1 \longrightarrow \text { antiquarks, } \\
& -q+1 \leq i \leq-1 \quad \longrightarrow \text { quarks, } \\
& 1 \leq i \leq n+2 \quad \longrightarrow \text { gluons. }
\end{aligned}
$$

The FKS parton will have label $-q$, and its sister label $-2 q$ (which implies that, for this case to be non trivial, these quarks must be massless). At the Born level, the gluon with label $n+2$ will be identified with the one branching into the $q \bar{q}$ pair. When describing the

\footnotetext{
${ }^{3}$ The label $i=0$ is not associated with any parton. In the following, I shall not bother to exclude explicitly $i=0$ when summing over parton labels; this condition will be understood.
} 
properties of a generic amplitude in the remainder of this section, I shall use the labelling of eqs. (3.6)-(3.8) to be definite.

The colours of gluons will be denoted as in eq. (2.3), whereas in the case of quarks and antiquarks:

$$
\left\{a_{i}\right\}_{i=-2 q}^{-1}, \quad a_{i} \in\{1, \ldots N\} .
$$

An amplitude at a given colour configuration, i.e. the analogue of eq. (2.4), will be written in the following way:

$$
\mathcal{A}^{(2 q ; n)}\left(a_{-2 q}, \ldots a_{n}\right)=\sum_{\gamma \in \mathcal{F}_{2 q ; n}} \Lambda\left(\left\{a_{i}\right\}, \gamma\right) \widehat{\mathcal{A}}^{(2 q ; n)}(\gamma),
$$

where $\gamma$ and $\mathcal{F}_{2 q ; n}$ play the same role as $\sigma$ and $P_{n}^{\prime}$ in eq. (2.4); namely, they denote a flow and the set of all flows relevant to the process of eq. (3.5) respectively. In order to determine the precise forms of these quantities, I use again the representation of ref. [1]. In particular, one sees that the generic form of the colour structure that multiplies the dual amplitude is:

$$
\begin{aligned}
\Lambda\left(\left\{a_{i}\right\}, \gamma\right)=N^{-\rho(\gamma)} & \left(\lambda^{a_{\sigma\left(t_{0}+1\right)}} \ldots \lambda^{a_{\sigma\left(t_{1}\right)}}\right)_{a_{-1} a_{\mu(-1-q)}} \\
& \times\left(\lambda^{a_{\sigma\left(t_{1}+1\right)}} \ldots \lambda^{\left.a_{\sigma\left(t_{2}\right)}\right)}\right)_{a_{-2} a_{\mu(-2-q)}} \\
& \times \ldots \\
& \times\left(\lambda^{a_{\sigma\left(t_{q-1}+1\right)}} \ldots \lambda^{a_{\sigma\left(t_{q}\right)}}\right)_{a_{-q} a_{\mu(-2 q)}}
\end{aligned}
$$

with

$$
\rho(\gamma)=\min \left\{q-1, \sum_{p=1}^{q} \delta(-p-q, \mu(-p-q))\right\} .
$$

In eq. (3.15), $\sigma$ and $\mu$ denote permutations (including cyclic ones) of the first $n$ and $q$ integers respectively, $\sigma \in P_{n}$ and $\mu \in P_{q}$. The range of the arguments of, and the values assumed by, the latter have been extended, so as:

$$
\begin{aligned}
\mu(p) & =-\mu(-p-q)-q, \quad-2 q \leq p \leq-q-1, \\
\Longrightarrow \quad \mu(p) & \in\{-q-1, \ldots-2 q\} .
\end{aligned}
$$

Finally, the set of $q+1$ integers $t_{p}$

$$
\begin{aligned}
t & =\left\{t_{0}, \ldots t_{q}\right\} \\
0 & =t_{0} \leq t_{1} \leq \ldots t_{q-1} \leq t_{q}=n
\end{aligned}
$$

achieves a partition of the ordered set of the first $n$ integers into $q$ subsets of ordered integers, with the $p^{\text {th }}$ cell of the partition defined to be:

$$
\left(t_{p-1}+1, t_{p-1}+2, \ldots t_{p}-1, t_{p}\right), \quad 1 \leq p \leq q .
$$

The ensemble of such partitions I shall denote by

$$
T_{n \mid q} \cdot
$$


It should be stressed that the $p^{\text {th }}$ cell of the partition, eq. (3.21), may coincide with the empty set, which one can formally write by setting

$$
t_{p-1}=t_{p} .
$$

This case of a zero-length cell corresponds to defining:

$$
\left(\lambda^{a_{\sigma\left(t_{p-1}+1\right)}} \ldots \lambda^{a_{\sigma\left(t_{p}\right)}}\right)_{a_{-p} a_{\mu(-p-q)}} \equiv \delta_{a_{-p} a_{\mu(-p-q)}}
$$

as prescribed in ref. [1]. Note that all cells of $t$ (in the case of amplitudes that feature only quarks), or all cells except one (when there is at least one gluon entering the process) can have zero length. Equation (3.15) implies the following definition of flow:

$$
\begin{aligned}
\gamma & =\bigcup_{p=1}^{q} \gamma_{p}, \\
\gamma_{p} & =\left(-p ; \sigma\left(t_{p-1}+1\right), \ldots \sigma\left(t_{p}\right) ; \mu(-p-q)\right) .
\end{aligned}
$$

The physical interpretation of these equations is straightforward. Equations (3.26) represents a colour antenna that connects the colour of quark $-p$ with the anticolour of antiquark $\mu(-p-q)$; attached to this line there are $t_{p}-t_{p-1}$ gluons (therefore, in the case of eq. (3.23), there will be no gluons). Then, the integer $\rho(\gamma)$, defined in eq. (3.16), is the number of times when a quark-antiquark colour line coincides with a flavour line (minus one, when there is a maximal coincidence). The flow, eq. (3.25), is the set of all colour lines. By convention, the $p^{\text {th }}$ colour line begins with quark $-p$. The construction above implies that

$$
\mathcal{F}_{2 q ; n}=\left(P_{n}, P_{q}, T_{n \mid q}\right) .
$$

At this point, one can trivially extend the definitions of colour vectors and the corresponding amplitudes given in section 2 . One has just to formally replace $1 \ldots n$ with $-2 q \ldots n$, and use the definition of flows given here rather than that of section 2. So for example

$$
\begin{aligned}
\left|\mathcal{A}^{(2 q ; n)}\left(a_{-2 q}, \ldots a_{n}\right)\right\rangle & =\mathcal{A}^{(2 q ; n)}\left(a_{-2 q}, \ldots a_{n}\right)\left|a_{-2 q}, \ldots a_{n}\right\rangle \\
\left|\mathcal{A}^{(2 q ; n)}(\gamma)\right\rangle & =\sum_{\left\{a_{i}\right\}_{i=-2 q}^{n}} \Lambda\left(\left\{a_{i}\right\}, \gamma\right) \widehat{\mathcal{A}}^{(2 q ; n)}(\gamma)\left|a_{-2 q}, \ldots a_{n}\right\rangle
\end{aligned}
$$

and so forth.

The case of amplitudes that feature more than one $q \bar{q}$ pair per (at least) one flavour is essentially identical to what has been discussed so far, and only requires additional information on flavour lines, which now are not unique. This information can be given e.g. in the following form:

$$
\begin{aligned}
f & =\bigcup_{p=1}^{q}\left(-p, f_{-p}\right), \\
\left\{f_{-1}, \ldots f_{-q}\right\} & =\{-q-1, \ldots-2 q\},
\end{aligned}
$$

where $\left(-p, f_{-p}\right)$ represents the flavour line that connects quark $-p$ with antiquark $f_{-p}$. Clearly, this notation encompasses the equal-flavour case as well, for which $f_{-p}=-p-q$ 
for all $p$ 's. At this point, one can define amplitudes at a fixed flavour configuration $f$, which are gauge invariant, and then sum over all these configurations to obtain the physical amplitude. The definition of the colour structure given in eq. (3.15) is unchanged, but eq. (3.16) needs be generalized to read:

$$
\rho(\gamma)=\min \left\{q-1, \sum_{p=1}^{q} \delta\left(f_{-p}, \mu(-p-q)\right)\right\} .
$$

The treatment of equal-flavour amplitudes does not pose any problems, but slightly complicates the notation. For this reason, in the following I shall deal explicitly only with the unequal-flavour case; the equal-flavour case can be easily recovered by adding a sum over the flavour configurations given in eq. (3.30).

\subsection{Subtraction of colour-summed matrix elements}

I shall extend here what was done in section 2.1 to the case of quark-gluon amplitudes squared. The formulae for the relevant soft and collinear limits are of course well known, and thus I shall limit myself here to casting them in a form suited to the calculations that I shall perform in the rest of this section. As already pointed out before, for quark-gluon amplitudes one needs distinguish the cases of the FKS parton being a gluon or a quark.

I start from the former case. The underlying Born dynamics is therefore that of eq. (3.5). The formulae for the soft limit of the amplitude squared are given in eqs. (2.30)(2.32), with only formal changes due to relabelling, and to the fact that self-eikonals need not vanish any longer, owing to the possible presence of massive quarks:

$$
\begin{aligned}
\mathcal{M}_{\mathrm{SOFT}}^{(2 q ; n+1)} & =\frac{1}{2} g_{S}^{2} \sum_{k, l=-2 q}^{n}[k, l] \mathcal{M}_{k l}^{(2 q ; n)}, \\
{[k, l] } & =\frac{k_{k} \cdot k_{l}}{k_{k} \cdot k_{n+1} k_{l} \cdot k_{n+1}}\left(1-\delta_{k l} \delta_{0 m_{k}}\right), \\
\mathcal{M}_{k l}^{(2 q ; n)} & =-2\left\langle\mathcal{A}^{(2 q ; n)}\left|\sum_{b} Q^{b}(k) Q^{b}(l)\right| \mathcal{A}^{(2 q ; n)}\right\rangle .
\end{aligned}
$$

The colour operators $Q^{b}(k)$ are defined in eqs. (2.33) and (2.34) in the case when $k>0$, i.e. when $k$ is a gluon. This definition needs be extended to the case of quarks and antiquarks. We have (see e.g. ref. [11]):

$$
\begin{aligned}
\left\langle a_{k} a_{n+1}\left|Q^{b}(k)\right| c_{k}\right\rangle & =\delta_{b a_{n+1}}\left(Q^{b}(k)\right)_{a_{k} c_{k}}, & & \\
\left(Q^{b}(k)\right)_{a_{k} c_{k}} & =\lambda_{a_{k} c_{k}}^{b} & -q \leq k \leq-1 & \Leftrightarrow k \text { is a quark, } \\
\left(Q^{b}(k)\right)_{a_{k} c_{k}} & =-\lambda_{c_{k} a_{k}}^{b} & -2 q \leq k \leq-q-1 & \Leftrightarrow k \text { is an antiquark. }
\end{aligned}
$$

With this, one can generalize eq. (2.38) to read:

$$
\begin{array}{rlrl}
\sum_{b} Q^{b}(k) Q^{b}(k) & =C(k) I, \\
C(k) & =C_{A} & & k>0, \\
C(k) & =C_{F} & & k<0 .
\end{array}
$$


The collinear limit can then be cast in the same form as in eq. (2.39):

$$
\begin{aligned}
\mathcal{M}_{\mathrm{COLL}}^{(2 q ; n+1)} & =\frac{g_{S}^{2}}{k_{s} \cdot k_{n+1}}\left\{\hat{P}_{\mathcal{I}_{s} \mathcal{I}_{s}}(z) \overline{\mathcal{M}}_{s}^{(2 q ; n)}+\hat{Q}_{\mathcal{I}_{s} \mathcal{I}_{s}^{\star}}(z) \Re\left(\frac{\left\langle k_{s} k_{n+1}\right\rangle}{\left[k_{s} k_{n+1}\right]} \overline{\mathcal{M}}_{s,-+}^{(2 q ; n)}\right)\right\}, \\
\overline{\mathcal{M}}_{s}^{(2 q ; n)} & =\left\langle\mathcal{A}^{(2 q ; n)}\left|\sum_{b} Q^{b}(s) Q^{b}(s)\right| \mathcal{A}^{(2 q ; n)}\right\rangle \\
\overline{\mathcal{M}}_{s,-+}^{(2 q ; n)} & =\left\langle\mathcal{A}_{-}^{(2 q ; n)}\left|\sum_{b} Q^{b}(s) Q^{b}(s)\right| \mathcal{A}_{+}^{(2 q ; n)}\right\rangle
\end{aligned}
$$

where $z$ is defined as in eq. (2.37), with $k_{n} \rightarrow k_{s}$ there. As was discussed at the beginning of section 3, I shall take

$$
s=n,-q,-2 q
$$

as representatives of the cases of the $g \rightarrow g g, q \rightarrow q g$, and $\bar{q} \rightarrow \bar{q} g$ branchings respectively. I denoted by $\mathcal{I}_{s}$ the identity of the relevant branching parton (i.e., a gluon, a quark, and an antiquark) and in eq. (3.42), consistently with eq. (2.42), I have defined:

$$
\hat{P}_{\mathcal{I}_{s} \mathcal{I}_{s}}(z)=\frac{1}{C(s)} P_{\mathcal{I}_{s} \mathcal{I}_{s}}(z), \quad \hat{Q}_{\mathcal{I}_{s} \mathcal{I}_{s}^{\star}}(z)=\frac{1}{C(s)} Q_{\mathcal{I}_{s} \mathcal{I}_{s}^{\star}}(z) .
$$

The consistency between eqs. (3.33) and (3.42), in the sense of eq. (2.29), can be proved in exactly the same way as was done in section 2, thanks to the fact that the colourconservation condition is fulfilled in the case of quark-gluon amplitudes as well (see appendix B):

$$
\sum_{k=-2 q}^{n} Q^{b}(k)\left|\mathcal{A}^{(2 q ; n)}\right\rangle=0 \Longrightarrow \sum_{\substack{k=-2 q \\ k \neq s}}^{n} Q^{b}(k)\left|\mathcal{A}^{(2 q ; n)}\right\rangle=-Q^{b}(s)\left|\mathcal{A}^{(2 q ; n)}\right\rangle
$$

and because eq. (2.47) generalizes to read:

$$
\hat{P}_{\mathcal{I}_{s} \mathcal{I}_{s}}(z) \stackrel{z \rightarrow 1}{\longrightarrow} \frac{2}{1-z}, \quad \hat{Q}_{\mathcal{I}_{s} \mathcal{I}_{s}^{\star}}(z) \stackrel{z \rightarrow 1}{\longrightarrow} 0,
$$

that is, it also holds in the case of $q \rightarrow q g$ and $\bar{q} \rightarrow \bar{q} g$ branchings. ${ }^{4}$

I shall now discuss the case of the $g \rightarrow q \bar{q}$ branchings. Here, the underlying Born is the process of eq. (3.9), and only the collinear limit is relevant (there are no soft singularities when the FKS parton is a quark). The analogues of eqs. (3.42)-(3.44) read:

$$
\begin{aligned}
& \mathcal{M}_{\mathrm{COLL}}^{(2 q ; n+1)}=\frac{g_{S}^{2}}{k_{-q} \cdot k_{-2 q}}\left\{\hat{P}_{q g}(z) \overline{\mathcal{M}}_{q q}^{(2(q-1) ; n+2)}\right. \\
&\left.+\hat{Q}_{q g^{\star}}(z) \Re\left(\frac{\left\langle k_{-q} k_{-2 q}\right\rangle}{\left[k_{-q} k_{-2 q}\right]} \overline{\mathcal{M}}_{q q,-+}^{(2(q-1) ; n+2)}\right)\right\}, \\
& \overline{\mathcal{M}}_{q q}^{(2(q-1) ; n+2)}=\left\langle\mathcal{A}^{(2(q-1) ; n+2)}\left|\sum_{b c} G_{b c}^{\star} G_{b c}\right| \mathcal{A}^{(2(q-1) ; n+2)}\right\rangle, \\
& \overline{\mathcal{M}}_{q q,-+}^{(2(q-1) ; n+2)}=\left\langle\mathcal{A}_{-}^{(2(q-1) ; n+2)}\left|\sum_{b c} G_{b c}^{\star} G_{b c}\right| \mathcal{A}_{+}^{(2(q-1) ; n+2)}\right\rangle,
\end{aligned}
$$

\footnotetext{
${ }^{4}$ For the branchings of quarks and antiquarks, the kernels $Q(z)$ are actually identical to zero [13].
} 
where

$$
\hat{P}_{q g}(z)=\frac{1}{T_{F}} P_{q g}(z), \quad \hat{Q}_{q g^{\star}}(z)=\frac{1}{T_{F}} Q_{q g^{\star}}(z) .
$$

The (non-hermitian) operators $G_{b c}$ are the analogues of the operators $Q^{b}$ introduced before. In colour space, their action is defined as follows:

$$
\left\langle a_{-2 q} a_{-q}\left|G_{b c}\right| a_{n+2}\right\rangle=\delta_{b a_{-q}} \delta_{c a_{-2 q}} \lambda_{a_{-q} a_{-2 q}}^{a_{n+2}} .
$$

In other words, the operators $G_{b c}$ annihilate gluon number $n+2$, and create the $q \bar{q}$ pair with labels $-q$ and $-2 q$ and colours $b$ and $c$ respectively. Note that eq. (3.49) does coincide with the usual form of the collinear limit since, as is easy to see from eq. (3.53), one has:

$$
\sum_{b c} G_{b c}^{\star} G_{b c}=T_{F} I
$$

which is the analogue of eq. (3.39).

\subsection{Subtraction at fixed colour configurations}

The procedure here is identical to that followed in section 2.2, except for trivial changes in notation. Its essence is that of inserting into the matrix element squared the projector onto a given colour configuration, that in this case reads:

$$
\left|a_{-2 q}, \ldots a_{n+1}\right\rangle\left\langle a_{-2 q}, \ldots a_{n+1}\right| .
$$

After doing that, one will also have to exploit the definitions of the $Q^{b}$ and $G_{b c}$ operators given in eqs. (3.36) and (3.53). The quantities whose soft and collinear limits one needs to construct are therefore

$$
\mathcal{M}^{(2 q ; n+1)}\left(a_{-2 q}, \ldots a_{n+1}\right)=\left|\mathcal{A}^{(2 q ; n+1)}\left(a_{-2 q}, \ldots a_{n+1}\right)\right|^{2} .
$$

Its soft limit can be obtained from eq. (3.33) through the procedure described above:

$$
\begin{gathered}
\mathcal{M}_{\mathrm{SOFT}}^{(2 q ; n+1)}\left(a_{-2 q}, \ldots a_{n+1}\right)=\frac{1}{2} g_{S}^{2} \sum_{k, l=-2 q}^{n}[k, l] \mathcal{M}_{k l}^{(2 q ; n)}\left(a_{-2 q}, \ldots a_{n+1}\right), \\
\mathcal{M}_{k l}^{(2 q ; n)}\left(a_{-2 q}, \ldots a_{n+1}\right)= \\
-2\left\langle\mathcal{A}^{(2 q ; n)}\left|Q^{a_{n+1}}(k)\right| a_{-2 q}, \ldots a_{n+1}\right\rangle\left\langle a_{-2 q}, \ldots a_{n+1}\left|Q^{a_{n+1}}(l)\right| \mathcal{A}^{(2 q ; n)}\right\rangle,
\end{gathered}
$$

which are fully analogous to eqs. (2.51) and (2.52). In terms of scalar quantities, eq. (3.58) gets rewritten as follows:

$$
\begin{aligned}
\mathcal{M}_{k l}^{(2 q ; n)}\left(a_{-2 q}, \ldots a_{n+1}\right)= & \\
-2 \sum_{b_{k}^{\prime} b_{l}} & \mathcal{A}^{(2 q ; n)}\left(a_{-2 q} \ldots b_{k}^{\prime} \ldots a_{l} \ldots a_{n}\right)^{\star} \mathcal{Q}_{\mathcal{I}_{k} \mathcal{I}_{l}}\left(a_{n+1} ; a_{k}, a_{l}\right)_{b_{k}^{\prime} b_{l}} \\
& \times \mathcal{A}^{(2 q ; n)}\left(a_{-2 q} \ldots a_{k} \ldots b_{l} \ldots a_{n}\right),
\end{aligned}
$$

with

$$
\mathcal{Q}_{\mathcal{I}_{k} \mathcal{I}_{l}}\left(a_{n+1} ; a_{k}, a_{l}\right)_{b c}=\left(Q^{a_{n+1}}(k)\right)_{b a_{k}}\left(Q^{a_{n+1}}(l)\right)_{a_{l} c} .
$$


This manifestly coincides with eq. (2.54) when $k$ and $l$ are both gluons. In the case of $k$ being a gluon and $l$ being a quark, in $\mathrm{SU}(3)$ there are $3 \cdot 8^{2} \mathcal{Q}$ matrices, of dimension $8 \times 3$ (for the case when the quark is on the left of the cut, and the gluon is on the right, the matrices have dimensions $3 \times 8$ ). When $k$ and $l$ are both quarks, the are $3^{2} \cdot 8 \mathcal{Q}$ matrices, of dimension $3 \times 3$. If quark(s) are replaced by antiquark(s), the number of matrices and their dimensions will not change, but their forms will (see eqs. (3.37) and (3.38)).

The case of the collinear limit is very similar. For $g \rightarrow g g, q \rightarrow q g$, and $\bar{q} \rightarrow \bar{q} g$ branchings, from eq. (3.42) one obtains:

$$
\begin{aligned}
\mathcal{M}_{\mathrm{COLL}}^{(2 q ; n+1)}\left(a_{-2 q}, \ldots a_{n+1}\right)=\frac{g_{S}^{2}}{k_{s} \cdot k_{n+1}} & \left\{\hat{P}_{\mathcal{I}_{s} \mathcal{I}_{s}}(z) \overline{\mathcal{M}}_{s}^{(2 q ; n)}\left(a_{-2 q}, \ldots a_{n+1}\right)\right. \\
& \left.+\hat{Q}_{\mathcal{I}_{s} \mathcal{I}_{s}^{\star}}(z) \Re\left(\frac{\left\langle k_{s} k_{n+1}\right\rangle}{\left[k_{s} k_{n+1}\right]} \overline{\mathcal{M}}_{s,-+}^{(2 q ; n)}\left(a_{-2 q}, \ldots a_{n+1}\right)\right)\right\},
\end{aligned}
$$

with

$$
\begin{aligned}
\overline{\mathcal{M}}_{s}^{(2 q ; n)}\left(a_{-2 q}, \ldots a_{n+1}\right)= & \left\langle\mathcal{A}^{(2 q ; n)}\left|Q^{a_{n+1}}(s)\right| a_{-2 q}, \ldots a_{n+1}\right\rangle \\
& \times\left\langle a_{-2 q}, \ldots a_{n+1}\left|Q^{a_{n+1}}(s)\right| \mathcal{A}^{(2 q ; n)}\right\rangle, \\
\overline{\mathcal{M}}_{s,-+}^{(2 q ; n)}\left(a_{-2 q}, \ldots a_{n+1}\right)= & \left\langle\mathcal{A}_{-}^{(2 q ; n)}\left|Q^{a_{n+1}}(s)\right| a_{-2 q}, \ldots a_{n+1}\right\rangle \\
& \times\left\langle a_{-2 q}, \ldots a_{n+1}\left|Q^{a_{n+1}}(s)\right| \mathcal{A}_{+}^{(2 q ; n)}\right\rangle .
\end{aligned}
$$

In terms of scalar quantities:

$$
\begin{aligned}
\overline{\mathcal{M}}_{s}^{(2 q ; n)}\left(a_{-2 q}, \ldots a_{n+1}\right)= & \\
& \sum_{b_{s}^{\prime} b_{s}} \mathcal{A}^{(2 q ; n)}\left(\left\{a_{i}\right\}_{i \neq s}, b_{s}^{\prime}\right)^{\star} \mathcal{Q}_{\mathcal{I}_{s} \mathcal{I}_{s}}\left(a_{n+1} ; a_{s}, a_{s}\right)_{b_{s}^{\prime} b_{s}} \mathcal{A}^{(2 q ; n)}\left(\left\{a_{i}\right\}_{i \neq s}, b_{s}\right), \\
\overline{\mathcal{M}}_{s,-+}^{(2 q ; n)}\left(a_{-2 q}, \ldots a_{n+1}\right)= & \\
& \sum_{b_{s}^{\prime} b_{s}} \mathcal{A}_{-}^{(2 q ; n)}\left(\left\{a_{i}\right\}_{i \neq s}, b_{s}^{\prime}\right)^{\star} \mathcal{Q}_{\mathcal{I}_{s} \mathcal{I}_{s}}\left(a_{n+1} ; a_{s}, a_{s}\right)_{b_{s}^{\prime} b_{s}} \mathcal{A}_{+}^{(2 q ; n)}\left(\left\{a_{i}\right\}_{i \neq s}, b_{s}\right) .
\end{aligned}
$$

The colour matrices that enter this equation are such that

$$
\sum_{a_{n+1}} \sum_{a_{s}} \mathcal{Q}_{\mathcal{I}_{s} \mathcal{I}_{s}}\left(a_{n+1} ; a_{s}, a_{s}\right)_{b c}=C(s) \delta_{b c}
$$

which extends eq. (2.60), and is the matrix form of eq. (3.39).

The case of the $g \rightarrow q \bar{q}$ collinear splitting can be derived starting from eq. (3.49). One obtains:

$$
\begin{aligned}
\mathcal{M}_{\mathrm{COLL}}^{(2 q ; n+1)}\left(a_{-2 q}, \ldots a_{n+1}\right)= & \frac{g_{S}^{2}}{k_{-q} \cdot k_{-2 q}}\left\{\hat{P}_{q g}(z) \overline{\mathcal{M}}_{q q}^{(2(q-1) ; n+2)}\left(a_{-2 q}, \ldots a_{n+1}\right)\right. \\
& \left.+\hat{Q}_{q g^{\star}}(z) \Re\left(\frac{\left\langle k_{-q} k_{-2 q}\right\rangle}{\left[k_{-q} k_{-2 q}\right]} \overline{\mathcal{M}}_{q q,-+}^{(2(q-1) ; n+2)}\left(a_{-2 q}, \ldots a_{n+1}\right)\right)\right\}
\end{aligned}
$$


where

$$
\begin{aligned}
\overline{\mathcal{M}}_{q q}^{(2(q-1) ; n+2)}\left(a_{-2 q}, \ldots a_{n+1}\right)= & \left\langle\mathcal{A}^{(2(q-1) ; n+2)}\left|G_{a_{-q} a_{-2 q}}^{\star}\right| a_{-2 q}, \ldots a_{n+1}\right\rangle \\
& \times\left\langle a_{-2 q}, \ldots a_{n+1}\left|G_{a_{-q} a_{-2 q}}\right| \mathcal{A}^{(2(q-1) ; n+2)}\right\rangle, \\
\overline{\mathcal{M}}_{q q,-+}^{(2(q-1) ; n+2)}\left(a_{-2 q}, \ldots a_{n+1}\right)= & \left\langle\mathcal{A}_{-}^{(2(q-1) ; n+2)}\left|G_{a_{-q} a_{-2 q}}^{\star}\right| a_{-2 q}, \ldots a_{n+1}\right\rangle \\
& \times\left\langle a_{-2 q}, \ldots a_{n+1}\left|G_{a_{-q} a_{-2 q}}\right| \mathcal{A}_{+}^{(2(q-1) ; n+2)}\right\rangle .
\end{aligned}
$$

In terms of scalar quantities, eqs. (3.68) and (3.69) read:

$$
\begin{aligned}
\overline{\mathcal{M}}_{q q}^{(2(q-1) ; n+2)}\left(a_{-2 q}, \ldots a_{n+1}\right)= & \sum_{b_{n+2}^{\prime} b_{n+2}} \mathcal{A}^{(2(q-1) ; n+2)}\left(a_{-2 q+1} \ldots a f_{q}, a_{-q+1} \ldots a_{n+1}, b_{n+2}^{\prime}\right)^{\star} \mathcal{G}\left(a_{-q}, a_{-2 q}\right)_{b_{n+2}^{\prime}} b_{n+2} \\
& \times \mathcal{A}^{(2(q-1) ; n+2)}\left(a_{-2 q+1} \ldots a a_{q}, a_{-q+1} \ldots a_{n+1}, b_{n+2}\right), \\
\overline{\mathcal{M}}_{q q,-+}^{(2(q-1) ; n+2)}\left(a_{-2 q}, \ldots a_{n+1}\right)= & \\
\sum_{b_{n+2}^{\prime} b_{n+2}} & \mathcal{A}_{-}^{(2(q-1) ; n+2)}\left(a_{-2 q+1} \ldots a / q, a_{-q+1} \ldots a_{n+1}, b_{n+2}^{\prime}\right)^{\star} \mathcal{G}\left(a_{-q}, a_{-2 q}\right)_{b_{n+2}^{\prime} b_{n+2}} \\
& \times \mathcal{A}_{+}^{(2(q-1) ; n+2)}\left(a_{-2 q+1} \ldots a-q, a_{-q+1} \ldots a_{n+1}, b_{n+2}\right),
\end{aligned}
$$

with

$$
\mathcal{G}\left(a_{-q}, a_{-2 q}\right)_{b c}=\lambda_{a_{-2 q} a_{-q}}^{b} \lambda_{a_{-q} a_{-2 q}}^{c} .
$$

As is clear from eq. (3.72), there are $3^{2} \mathcal{G}$ matrices, of dimensions $8 \times 8$ in $\mathrm{SU}(3)$. They are such that

$$
\sum_{a_{-q}} \sum_{a_{-2 q}} \mathcal{G}\left(a_{-q}, a_{-2 q}\right)_{b c}=T_{F} \delta_{b c}
$$

which is the matrix form of eq. (3.54).

\subsection{Subtraction at fixed flows}

Although slightly more complicated than in the case of gluon amplitudes owing to the presence of quarks, the derivation of the subtraction at fixed flows for quark-gluon amplitudes proceeds exactly as that carried out in section 2.3. More specifically, I shall use the expressions of the soft and collinear limits of the colour-summed matrix elements squared, and expand them in terms of either the real or the Born flows, using fixed-flows amplitudes and the properties of the colour operators $Q^{b}$ and $G_{b c}$. All the necessary technical ingredients are reported in appendix B.2. There, I extend (if necessary) the definitions and derivations of appendix B.1, relevant to gluon amplitudes, in a way which renders it straightforward the use of arguments by analogy.

There is however one type of singular limit of the quark-gluon amplitudes that does not have an analogue in the case of gluon amplitudes, namely that due to $g \rightarrow q \bar{q}$ branchings. I shall deal with these singularities in section 3.3.3. 


\subsubsection{Fixed real flows}

The quantity whose limits at given real flows I seek to construct is the analogue of that in eq. (2.62), namely:

$$
\begin{aligned}
\mathcal{M}^{(2 q ; n+1)}\left(\Gamma^{\prime}, \Gamma\right) & =\left\langle\mathcal{A}^{(2 q ; n+1)}\left(\Gamma^{\prime}\right) \mid \mathcal{A}^{(2 q ; n+1)}(\Gamma)\right\rangle \\
& =\widehat{\mathcal{A}}^{(2 q ; n+1)}\left(\Gamma^{\prime}\right)^{\star} C\left(\Gamma^{\prime}, \Gamma\right) \widehat{\mathcal{A}}^{(2 q ; n+1)}(\Gamma),
\end{aligned}
$$

where (see eq. (3.27)):

$$
\Gamma, \Gamma^{\prime} \in \mathcal{F}_{2 q ; n+1}=\left(P_{n+1}, P_{q}, T_{n+1 \mid q}\right),
$$

and the colour-flow matrix elements are defined analogously to eq. (2.21):

$$
C\left(\Gamma^{\prime}, \Gamma\right)=\sum_{\left\{a_{i}\right\}_{i=-2 q}^{n+1}} \Lambda\left(\left\{a_{i}\right\}, \Gamma^{\prime}\right)^{\star} \Lambda\left(\left\{a_{i}\right\}, \Gamma\right) .
$$

As was done in section 2.3.1, one begins by introducing real-emission-level colour vectors associated with an underlying Born dynamics:

$$
\left|\mathcal{A}_{\mathrm{EXT}}^{(2 q ; n)}(\Gamma)\right\rangle=\sum_{\left\{a_{i}\right\}_{i=-2 q}^{n+1}} \Lambda\left(\left\{a_{i}\right\}, \Gamma\right) \widehat{\mathcal{A}}^{(2 q ; n)}\left(\Gamma_{\not+\mathcal{I}}\right)\left|a_{-2 q}, \ldots a_{n+1}\right\rangle .
$$

The Born-level flow $\Gamma_{n+千}$ is obtained from the real-level one $\Gamma$ by eliminating from it the $(n+1)^{\text {th }}$ gluon. More precisely, if

$$
\Gamma=(\Sigma, M, T) \in \mathcal{F}_{2 q ; n+1}, \quad T=\left\{T_{0}, \ldots T_{q}\right\},
$$

then

$$
\Gamma_{\text {p+士 }}=(\sigma, \mu, t) \in \mathcal{F}_{2 q ; n},
$$

where

$$
\begin{aligned}
\sigma & \equiv(\sigma(1), \ldots \sigma(n))=(\Sigma(1), \ldots n+1, \ldots \Sigma(n+1)), \\
\mu & =M \\
t & =\left\{t_{0}, \ldots t_{q}\right\}
\end{aligned}
$$

with

$$
\begin{array}{lll}
t_{p}=T_{p} & \forall p \text { s.t. } T_{p}+1 \leq \Sigma^{-1}(n+1), \\
t_{p}=T_{p}-1 & & \text { otherwise. }
\end{array}
$$

Equation (3.80) is the same as in the case of gluon amplitudes - the relative ordering of the gluons has nothing to do with their belonging to a given colour line. Eq. (3.80) states the obvious but crucial fact that by removing one gluon the structure of the colour lines is not affected. Eqs. (3.80)-(3.84) imply that all colour lines in $\Gamma_{n+千}$ contain exactly the same particles and in the same order as those in $\Gamma$. The exception is the colour line derived from the colour line $\Gamma_{r} \in \Gamma$ that contains gluon $n+1$, since the latter gluon must be removed by 
definition; the remaining particles on that line, however, have the same relative ordering as in $\Gamma_{r}$.

At this point, as was done in section 2.3.1 I start by expressing the soft limit of the matrix element squared as a sum over Born-level flows. The analogues of eqs. (2.68)-(2.70) read as follows:

$$
\begin{aligned}
\mathcal{M}_{\mathrm{SOFT}}^{(2 q ; n+1)} & =\sum_{\gamma, \gamma^{\prime} \in \mathcal{F}_{2 q ; n}} \mathcal{M}_{\mathrm{SOFT}}^{(2 q ; n+1)}\left(\gamma^{\prime}, \gamma\right) \\
\mathcal{M}_{\mathrm{SOFT}}^{(2 q ; n+1)}\left(\gamma^{\prime}, \gamma\right) & =\frac{1}{2} g_{S}^{2} \sum_{k, l=-2 q}^{n}[k, l] \mathcal{M}_{k l}^{(2 q ; n)}\left(\gamma^{\prime}, \gamma\right) \\
\mathcal{M}_{k l}^{(2 q ; n)}\left(\gamma^{\prime}, \gamma\right) & =-2\left\langle\mathcal{A}^{(2 q ; n)}\left(\gamma^{\prime}\right)\left|\sum_{b} Q^{b}(k) Q^{b}(l)\right| \mathcal{A}^{(2 q ; n)}(\gamma)\right\rangle .
\end{aligned}
$$

The colour-linked Born's of eq. (3.87) can be computed using the results of appendix B.2. In particular, from eqs. (B.34)-(B.36) one obtains:

$$
\begin{aligned}
\sum_{b} Q^{b}(l)\left|\mathcal{A}^{(2 q ; n)}(\gamma)\right\rangle & =\left|\mathcal{A}_{\mathrm{EXT}}^{(2 q ; n)}\left(I_{+}\left(\gamma^{-1}(l)\right) \gamma\right)\right\rangle-\left|\mathcal{A}_{\mathrm{EXT}}^{(2 q ; n)}\left(I_{-}\left(\gamma^{-1}(l)\right) \gamma\right)\right\rangle \\
\sum_{b} Q^{b}(l)\left|\mathcal{A}^{(2 q ; n)}(\gamma)\right\rangle & =\left|\mathcal{A}_{\mathrm{EXT}}^{(2 q ; n)}\left(I_{+}\left(\gamma^{-1}(l)\right) \gamma\right)\right\rangle \\
\sum_{b} Q^{b}(l)\left|\mathcal{A}^{(2 q ; n)}(\gamma)\right\rangle & =-\left|\mathcal{A}_{\mathrm{EXT}}^{(2 q ; n)}\left(I_{-}\left(\gamma^{-1}(l)\right) \gamma\right)\right\rangle
\end{aligned}
$$

for the cases when $l$ is a gluon $(1 \leq l \leq n)$, a quark $(-q \leq l \leq-1)$, or an antiquark $(-2 q \leq l \leq-q-1)$ respectively. I have denoted by $\gamma^{-1}(l)$ the position of particle $l$ in the list that defines the colour flow (see appendix B.2, also for the definitions of the operators $\left.I_{ \pm}\right)$. One can now plug eqs. (3.88)-(3.90) into eq. (3.87) and proceed to the explicit expansion of that equation. This calculation can be performed using the very same method as in appendix B.2, since the colour structure of the sums over $k$ or $l$ is the same as that relevant to colour conservation. More explicitly, one starts by splitting the sum over particle labels into sums relevant to single colour lines, and then converts sums over particle labels into sums over particle positions. This implies the following manipulations (where I consider the sum over $l$ to give a definite example):

$$
\begin{aligned}
\sum_{l=-2 q}^{n} f(l) & =\sum_{p=1}^{q} \sum_{l \in \gamma_{p}} f(l)=\sum_{p=1}^{q}\left(f(-p)+\sum_{l \in \gamma_{p}} f(l) \delta_{\mathcal{I}_{l} g}+f(\mu(-p-q))\right) \\
& =\sum_{p=1}^{q}\left(\delta_{j(-p)} f(\gamma(j))+\sum_{j=t_{p-1}+1}^{t_{p}} f(\gamma(j))+\delta_{j(-p-q)} f(\gamma(j))\right),
\end{aligned}
$$

where the three terms on the r.h.s.'s of eqs. (3.91) and (3.92) correspond to the quark, gluons, and antiquark contributions respectively, relevant to the $p^{\text {th }}$ colour line. The arguments of $f()$ in eq. (3.92) imply that the operators $I_{ \pm}\left(\gamma^{-1}(\gamma(j))\right)=I_{ \pm}(j)$ will result from eqs. (3.88)-(3.90). For each colour line, there will be $t_{p}-t_{p-1}+1$ contributions due to $I_{+}$operators (from the gluons and the quark), and $t_{p}-t_{p-1}+1$ contributions due to 
$I_{-}$operators (from the gluons and the antiquark). Furthermore, the $I_{-}$operators can be rewritten in terms of $I_{+}$operators by using the identity in eq. (B.40). After some trivial algebra, fully analogue to that of eqs. (B.39)-(B.42) bar the presence of the eikonal factors in eq. (3.86), one obtains:

$$
\begin{aligned}
\mathcal{M}_{\mathrm{SOFT}}^{(2 q ; n+1)}\left(\gamma^{\prime}, \gamma\right)=-g_{S}^{2} \sum_{p^{\prime}, p=1}^{q} & \sum_{i=t_{p^{\prime}-1}+1 \ominus 1}^{t_{p^{\prime}}} \sum_{j=t_{p-1}+1 \ominus 1}^{t_{p}}\left\{\left[\gamma^{\prime}(i), \gamma(j)\right]-\left[\gamma^{\prime}(i), \gamma(j \oplus 1)\right]\right. \\
& \left.-\left[\gamma^{\prime}(i \oplus 1), \gamma(j)\right]+\left[\gamma^{\prime}(i \oplus 1), \gamma(j \oplus 1)\right]\right\} \\
& \times\left\langle\mathcal{A}_{\mathrm{EXT}}^{(2 q ; n)}\left(I_{+}(i) \gamma^{\prime}\right) \mid \mathcal{A}_{\mathrm{EXT}}^{(2 q ; n)}\left(I_{+}(j) \gamma\right)\right\rangle .
\end{aligned}
$$

This result is basically identical to that of eq. (2.78), which can be easily understood as follows (for the sake of this argument, it is sufficient to consider the case of flows with one colour line). In a gluon amplitude, the $(n+1)^{\text {th }}$ gluon appears in a given position in a real-level flow as the result of the actions of the operators $I_{+}$and $I_{-}$associated with the gluon that precedes and follows it in the flow respectively. When the $(n+1)^{\text {th }}$ gluon occupies the first (last) position, the relevant $I_{+}\left(I_{-}\right)$operator is that associated with the last (first) gluon in the flow, as a consequence of the cyclicity of the trace. In the case of quark-gluon amplitudes, the colour structure is not cyclic, so the latter argument is not valid. However, when the $(n+1)^{\text {th }}$ gluon is the left-most (right-most) gluon of the flow, there exists a relevant $I_{+}\left(I_{-}\right)$operator, associated with the quark (antiquark) rather than with the last (first) gluon in the flow. It should finally be stressed that the fact that the quantities $i+1$ and $j+1$ which appear in eq. (2.78) are replaced by $i \oplus 1$ and $j \oplus 1$ in eq. (3.93) is only a formal difference. The physical meaning is in fact the same: in both cases, these denote the particles to the immediate right of particles $i$ and $j$.

Equation (3.93) is in a form suited to transform the sum in eq. (3.85) in a sum over real flows. The procedure is identical to that of section 2.3.1 and, as in that case, stems from the observation that there is a one-to-one correspondence between a real flow $\Gamma$, and a pair $(\gamma, j)$, where $\gamma$ is a Born flow, and $j$ is an integer whose range is the same as that in eq. (3.93). This follows from the existence and uniqueness of the solution of the equation:

$$
\begin{aligned}
I_{+}(j) \gamma=\Gamma \Longleftrightarrow & \\
& \ldots(\ldots \gamma(j), n+1, \gamma(j \oplus 1), \ldots) \ldots \\
=\ldots(\ldots \Gamma(j), \Gamma(j \oplus 1), \Gamma(j \oplus 1 \oplus 1), \ldots) \ldots, &
\end{aligned}
$$

as was the case for its analogue, eq. (2.82) or eq. (2.83). The use of the $\oplus$ operator in eq. (3.95) in place of the ordinary + that appears in eq. (2.83) does not underscore any difference between the two cases, but simply allows one to treat with the same notation the cases in which $j$ is a gluon or is a quark (note that the range spanned by $j$ in eq. (3.93) implies that $j$ is never an antiquark). The existence and uniqueness of the solution of eq. (3.95) can be easily understood e.g. from the relationships that connect real- and Bornlevel flows according to eqs. (3.80)-(3.84). Furthermore, one has to take into account the fact that for the two flows $\gamma_{a}=\left(\sigma_{a}, \mu_{a}, t_{a}\right)$ and $\gamma_{b}=\left(\sigma_{b}, \mu_{b}, t_{b}\right)$ to be different, it is sufficient 
that only one of the conditions $\sigma_{a} \neq \sigma_{b} ; \mu_{a} \neq \mu_{b} ; t_{a} \neq t_{b}$ be fulfilled. By solving eq. (3.95) one obtains:

$$
\begin{aligned}
& \gamma=\Gamma_{n+1}, \\
& j=\Gamma^{-1}(n+1) \ominus 1,
\end{aligned}
$$

which also imply that:

$$
\begin{aligned}
\gamma(j) & =\Gamma\left(\Gamma^{-1}(n+1) \ominus 1\right), \\
\gamma(j \oplus 1) & =\Gamma\left(\Gamma^{-1}(n+1) \oplus 1\right) .
\end{aligned}
$$

In full analogy with eq. (2.86), the arguments above are such that:

$$
\sum_{\gamma \in \mathcal{F}_{2 q ; n}} \sum_{p=1}^{q} \sum_{j=t_{p-1}+1 \ominus 1}^{t_{p}} f(\gamma, j) \equiv \sum_{\Gamma \in \mathcal{F}_{2 q ; n+1}} f\left(\Gamma_{p+\Psi}, \Gamma^{-1}(n+1) \ominus 1\right) .
$$

As a consistency check of eq. (3.100), one can again use a counting argument as done in the case of gluon amplitudes. Denoting by $\mathcal{N}(n, q)$ the number of partitions defined in eqs. (3.19) and (3.20), one can prove that:

$$
\mathcal{N}(n, q)=\left(\begin{array}{c}
n+q-1 \\
q-1
\end{array}\right)
$$

The number of terms that appear in the sums on the l.h.s. of eq. (3.100) is

$$
q ! n ! \mathcal{N}(n, q)(n+q)
$$

since there are $(n+q)$ different ways of inserting a gluon in a Born flow $(n+q$ is in fact the number of terms of the sums over $p$ and $j$ in eq. (3.100)). On the r.h.s. of eq. (3.100) the number of terms is instead:

$$
q !(n+1) ! \mathcal{N}(n+1, q) .
$$

Thanks to eq. (3.101), one sees that the numbers in eqs. (3.102) and (3.103) coincide.

By using the results above, their analogues for the L-flows, and eq. (3.93), one finally obtains the soft limit of the matrix element squared at fixed real flows:

$$
\begin{aligned}
& \mathcal{M}_{\mathrm{SOFT}}^{(2 q ; n+1)}= \sum_{\Gamma, \Gamma^{\prime} \in \mathcal{F}_{2 q ; n+1}} \mathcal{M}_{\mathrm{SOFT}}^{(2 q ; n+1)}\left(\Gamma^{\prime}, \Gamma\right) \\
& \mathcal{M}_{\mathrm{SOFT}}^{(2 q ; n+1)}\left(\Gamma^{\prime}, \Gamma\right)=-g_{S}^{2}\left\{\left[\Gamma^{\prime}\left(\Gamma^{\prime-1}(n+1) \ominus 1\right), \Gamma\left(\Gamma^{-1}(n+1) \ominus 1\right)\right]\right. \\
& \quad-\left[\Gamma^{\prime}\left(\Gamma^{\prime-1}(n+1) \ominus 1\right), \Gamma\left(\Gamma^{-1}(n+1) \oplus 1\right)\right] \\
& \quad-\left[\Gamma^{\prime}\left(\Gamma^{\prime-1}(n+1) \oplus 1\right), \Gamma\left(\Gamma^{-1}(n+1) \ominus 1\right)\right] \\
&\left.\quad+\left[\Gamma^{\prime}\left(\Gamma^{\prime-1}(n+1) \oplus 1\right), \Gamma\left(\Gamma^{-1}(n+1) \oplus 1\right)\right]\right\} \mathcal{M}_{\mathrm{EXT}}^{(2 q ; n)}\left(\Gamma^{\prime}, \Gamma\right) \\
& \mathcal{M}_{\mathrm{EXT}}^{(2 q ; n)}\left(\Gamma^{\prime}, \Gamma\right)=\left\langle\mathcal{A}_{\mathrm{EXT}}^{(2 q ; n)}\left(\Gamma^{\prime}\right) \mid \mathcal{A}_{\mathrm{EXT}}^{(2 q ; n)}(\Gamma)\right\rangle
\end{aligned}
$$


The form of eq. (3.105) is fully analogous to that of eq. (2.90). In terms of scalar quantities:

$$
\mathcal{M}_{\mathrm{EXT}}^{(2 q ; n)}\left(\Gamma^{\prime}, \Gamma\right)=\widehat{\mathcal{A}}^{(2 q ; n)}\left(\Gamma_{n+1}^{\prime}\right)^{\star} C\left(\Gamma^{\prime}, \Gamma\right) \widehat{\mathcal{A}}^{(2 q ; n)}\left(\Gamma_{n+1}\right) .
$$

The derivation of the soft limit of the matrix elements given above shows the complete similarity between the cases of gluon and quark-gluon amplitudes. The collinear limits relevant to the branchings $g \rightarrow g g, q \rightarrow q g$, and $\bar{q} \rightarrow \bar{q} g$ are also fully analogous, as one can easily understand by comparing eqs. (2.39)-(2.41) with eqs. (3.42)-(3.44). In other words, in the case of quark-gluon amplitudes one can follow the procedure that in section 2.3.1 has led me from eq. (2.95) to eqs. (2.109)-(2.111). I refrain from doing that explicitly, and limit myself to present the final result:

$$
\begin{aligned}
\mathcal{M}_{\mathrm{COLL}}^{(2 q ; n+1)}= & \sum_{\Gamma, \Gamma^{\prime} \in \mathcal{F}_{2 q ; n+1}} \mathcal{M}_{\mathrm{COLL}}^{(2 q ; n+1)}\left(\Gamma^{\prime}, \Gamma\right), \\
\mathcal{M}_{\mathrm{COLL}}^{(2 q ; n+1)}\left(\Gamma^{\prime}, \Gamma\right)= & \frac{g_{S}^{2}}{k_{s} \cdot k_{n+1}} \delta\left(\Gamma^{\prime}, \Gamma\right)\left\{\hat{P}_{\mathcal{I}_{s} \mathcal{I}_{s}}(z) \mathcal{M}_{\mathrm{EXT}}^{(2 q ; n)}\left(\Gamma^{\prime}, \Gamma\right)\right. \\
& \left.+\frac{1}{2} \hat{Q}_{\mathcal{I}_{s} \mathcal{I}_{s}^{\star}}(z)\left(\frac{\left\langle k_{s} k_{n+1}\right\rangle}{\left[k_{s} k_{n+1}\right]} \mathcal{M}_{\mathrm{EXT}-+}^{(2 q ; n)}\left(\Gamma^{\prime}, \Gamma\right)+\frac{\left[k_{s} k_{n+1}\right]}{\left\langle k_{s} k_{n+1}\right\rangle} \mathcal{M}_{\mathrm{EXT}+-}^{(2 q ; n)}\left(\Gamma^{\prime}, \Gamma\right)\right)\right\},
\end{aligned}
$$

where

$$
\mathcal{M}_{\mathrm{EXT} \lambda \bar{\lambda}}^{(2 q ; n)}\left(\Gamma^{\prime}, \Gamma\right)=\left\langle\mathcal{A}_{\mathrm{EXT \lambda}}^{(2 q ; n)}\left(\Gamma^{\prime}\right) \mid \mathcal{A}_{\mathrm{EXT} \bar{\lambda}}^{(2 q ; n)}(\Gamma)\right\rangle
$$

and

$$
\delta\left(\Gamma^{\prime}, \Gamma\right)=\sum_{\alpha=-1,1} \sum_{\beta=-1,1} \alpha \beta \delta\left(\Gamma^{\prime^{-1}}(s), \Gamma^{\prime-1}(n+1) \oplus \alpha\right) \delta\left(\Gamma^{-1}(s), \Gamma^{-1}(n+1) \oplus \beta\right) .
$$

In terms of scalar quantities:

$$
\mathcal{M}_{\mathrm{EXT \lambda \overline { \lambda }}}^{(2 q ; n)}\left(\Gamma^{\prime}, \Gamma\right)=\widehat{\mathcal{A}}_{\lambda}^{(2 q ; n)}\left(\Gamma_{n+千}^{\prime}\right)^{\star} C\left(\Gamma^{\prime}, \Gamma\right) \widehat{\mathcal{A}}_{\bar{\lambda}}^{(2 q ; n)}\left(\Gamma_{n+千}\right) .
$$

The very close correspondence between eqs. (3.105) and (3.109), and their counterparts in the case of gluon amplitudes, implies that the consistency (in the sense of eq. (2.29)) between the soft and collinear limits of quark-gluon matrix elements can be proved in exactly the same way as explained at the end of section 2.3.1.

One comment is in order as far as the form of eq. (3.111) is concerned. In the case of a $g \rightarrow g g$ branching, i.e. when $s=n$, the situation is strictly identical to that explicitly presented in eqs. (2.100)-(2.102). However, when one considers a $q \rightarrow q g$ branching, i.e. when $s=-q$, the analogue of eq. (2.100) contains only one term, because of eq. (3.89). Therefore, the relevant real flows, which correspond to those in eqs. (2.101) and (2.102), are:

$$
I_{+}\left(\gamma^{\prime^{-1}}(-q)\right) \gamma^{\prime}, \quad I_{+}\left(\gamma^{-1}(-q)\right) \gamma \longrightarrow(-q ; n+1, \ldots) .
$$

These correspond to the contribution obtained when $\alpha=-1$ and $\beta=-1$ in eq. (3.111). However, it is easy to see that if either $\alpha=1$ or $\beta=1$ in eq. (3.111), then $\delta\left(\Gamma^{\prime}, \Gamma\right)=0$ if $s=-q$. In fact, if $\delta\left(\Gamma^{\prime}, \Gamma\right)=1$ these cases would correspond to real flows where the $(n+1)^{\text {th }}$ gluon occupied the position at the immediate left of quark $-q$, which is impossible. Similar arguments apply to the $\bar{q} \rightarrow \bar{q} g$ branching, i.e. when $s=-2 q$. The bottom line is that eq. (3.111) can indeed be used to describe all branchings considered here, as claimed before. 


\subsubsection{Fixed Born flows}

The results relevant to fixing the Born flows can be straightforwardly obtained from section 3.3.1. Once again, the analogy with the case of gluon amplitudes is very close.

The soft limit of the matrix element has already been presented in eqs. (3.85)-(3.87). The real-emission-level quantity whose soft limit is $\mathcal{M}_{\text {SOFT }}^{(2 q ; n+1)}\left(\gamma^{\prime}, \gamma\right)$ will be a linear combination of the fixed-real-flow matrix elements $\mathcal{M}^{(2 q ; n+1)}\left(\Gamma^{\prime}, \Gamma\right)$ defined in eq. (3.74):

$$
\mathcal{M}^{(2 q ; n+1)}\left(\gamma^{\prime}, \gamma\right)=\sum_{\Gamma \in \xi(\gamma)} \sum_{\Gamma^{\prime} \in \xi\left(\gamma^{\prime}\right)} \mathcal{M}^{(2 q ; n+1)}\left(\Gamma^{\prime}, \Gamma\right),
$$

for suitable sets of real flows $\xi(\gamma)$ and $\xi\left(\gamma^{\prime}\right)$, to be defined later. In terms of scalar quantities, the colour-linked Born's of eq. (3.87) read:

$$
\begin{aligned}
\mathcal{M}_{k l}^{(2 q ; n)}\left(\gamma^{\prime}, \gamma\right)=-2 \widehat{\mathcal{A}}^{(2 q ; n)}\left(\gamma^{\prime}\right)^{\star}[ & C\left(I_{+}\left(\gamma^{\prime-1}(k)\right) \gamma^{\prime}, I_{+}\left(\gamma^{-1}(l)\right) \gamma\right)\left(1-\delta_{\bar{q} \mathcal{I}_{k}}\right)\left(1-\delta_{\bar{q} \mathcal{I}_{l}}\right) \\
& -C\left(I_{+}\left(\gamma^{\prime-1}(k)\right) \gamma^{\prime}, I_{-}\left(\gamma^{-1}(l)\right) \gamma\right)\left(1-\delta_{\bar{q} \mathcal{I}_{k}}\right)\left(1-\delta_{q \mathcal{I}_{l}}\right) \\
& -C\left(I_{-}\left(\gamma^{\prime-1}(k)\right) \gamma^{\prime}, I_{+}\left(\gamma^{-1}(l)\right) \gamma\right)\left(1-\delta_{q \mathcal{I}_{k}}\right)\left(1-\delta_{\bar{q} \mathcal{I}_{l}}\right) \\
& \left.+C\left(I_{-}\left(\gamma^{\prime-1}(k)\right) \gamma^{\prime}, I_{-}\left(\gamma^{-1}(l)\right) \gamma\right)\left(1-\delta_{q \mathcal{I}_{k}}\right)\left(1-\delta_{q \mathcal{I}_{l}}\right)\right] \\
& \times \widehat{\mathcal{A}}^{(2 q ; n)}(\gamma) .
\end{aligned}
$$

The $(1-\delta)$ terms that multiply the colour-flow matrix elements in the r.h.s. of eq. (3.115) exclude the contributions of the $I_{-}$and $I_{+}$operators in the case when the corresponding particle is a quark or an antiquark respectively, as dictated by eqs. (3.89) and (3.90).

Equation (3.114) is the analogue of eq. (2.117), and the set $\xi(\gamma)$ is the analogue of its counterpart $\zeta(\sigma)$ in the case of gluon amplitudes, defined in eq. (2.114). Since $\zeta(\sigma)$ is the set of all real flows that can be obtained by acting on a given Born flow $\sigma$ with the operators $I_{ \pm}$relevant to the computation of the colour-linked Born's, one can obtain $\xi(\gamma)$ precisely in the same way. Hence, from eqs. (3.87)-(3.90), one gets:

$$
\begin{aligned}
\xi(\gamma) & =\bigcup_{l=1}^{n}\left\{I_{+}\left(\gamma^{-1}(l)\right) \gamma, I_{-}\left(\gamma^{-1}(l)\right) \gamma\right\} \bigcup_{l=-q}^{-1}\left\{I_{+}\left(\gamma^{-1}(l)\right) \gamma\right\} \bigcup_{l=-2 q}^{-q-1}\left\{I_{-}\left(\gamma^{-1}(l)\right) \gamma\right\} \\
& =\bigcup_{p=1}^{q} \bigcup_{j=t_{p-1}+1 \ominus 1}^{t_{p}}\left\{I_{+}(j) \gamma\right\},
\end{aligned}
$$

where the last form follows from eq. (B.40), but could equally well be deduced directly from eqs (3.88)-(3.93). The derivation presented in section 3.3.1 implies that:

$$
\begin{aligned}
\xi\left(\gamma_{1}\right) \bigcap \xi\left(\gamma_{2}\right) & =\emptyset \quad \text { if } \gamma_{1} \neq \gamma_{2} \\
\bigcup_{\gamma \in \mathcal{F}_{2 q ; n}} \xi(\gamma) & =\mathcal{F}_{2 q ; n+1}
\end{aligned}
$$

as in eqs. (2.115) and (2.116). Hence:

$$
\mathcal{M}^{(2 q ; n+1)}=\sum_{\gamma, \gamma^{\prime} \in \mathcal{F}_{2 q ; n}} \mathcal{M}^{(2 q ; n+1)}\left(\gamma^{\prime}, \gamma\right),
$$


which confirms that indeed the quantity defined eq. (3.114) has the property that one expects.

In the collinear limit, the matrix elements at fixed Born flows can be obtained directly from eqs. (3.42)-(3.44), by using the identity of eq. (3.39):

$$
\begin{aligned}
\mathcal{M}_{\mathrm{COLL}}^{(2 q ; n+1)}\left(\gamma^{\prime}, \gamma\right)= & \frac{g_{S}^{2}}{k_{s} \cdot k_{n+1}}\left\{P_{\mathcal{I}_{s} \mathcal{I}_{s}}(z) \mathcal{M}^{(2 q ; n)}\left(\gamma^{\prime}, \gamma\right)\right. \\
& \left.+\frac{1}{2} Q_{\mathcal{I}_{s} \mathcal{I}_{s}^{\star}}(z)\left(\frac{\left\langle k_{s} k_{n+1}\right\rangle}{\left[k_{s} k_{n+1}\right]} \mathcal{M}_{-+}^{(2 q ; n)}\left(\gamma^{\prime}, \gamma\right)+\frac{\left[k_{s} k_{n+1}\right]}{\left\langle k_{s} k_{n+1}\right\rangle} \mathcal{M}_{+-}^{(2 q ; n)}\left(\gamma^{\prime}, \gamma\right)\right)\right\},
\end{aligned}
$$

with the reduced matrix elements that have the usual definitions:

$$
\begin{aligned}
\mathcal{M}^{(2 q ; n)}\left(\gamma^{\prime}, \gamma\right) & =\left\langle\mathcal{A}^{(2 q ; n)}\left(\gamma^{\prime}\right) \mid \mathcal{A}^{(2 q ; n)}(\gamma)\right\rangle \\
& =\widehat{\mathcal{A}}^{(2 q ; n)}\left(\gamma^{\prime}\right)^{\star} C\left(\gamma^{\prime}, \gamma\right) \widehat{\mathcal{A}}^{(2 q ; n)}(\gamma), \\
\mathcal{M}_{\lambda \bar{\lambda}}^{(2 q ; n)}\left(\gamma^{\prime}, \gamma\right) & =\left\langle\mathcal{A}_{\lambda}^{(2 q ; n)}\left(\gamma^{\prime}\right) \mid \mathcal{A}_{\bar{\lambda}}^{(2 q ; n)}(\gamma)\right\rangle \\
& =\widehat{\mathcal{A}}_{\lambda}^{(2 q ; n)}\left(\gamma^{\prime}\right)^{\star} C\left(\gamma^{\prime}, \gamma\right) \widehat{\mathcal{A}}_{\bar{\lambda}}^{(2 q ; n)}(\gamma) .
\end{aligned}
$$

The very same arguments as in the case of gluon amplitudes can now be applied. Namely, one can construct the sets

$$
\xi_{\mathrm{C}}(\gamma)=\left\{I_{+}\left(\gamma^{-1}(n)\right) \gamma, I_{-}\left(\gamma^{-1}(n)\right) \gamma\right\}, \quad\left\{I_{+}\left(\gamma^{-1}(-q)\right)\right\}, \quad\left\{I_{-}\left(\gamma^{-1}(-2 q)\right)\right\}
$$

relevant to the $g \rightarrow g g, q \rightarrow q g$, and $\bar{q} \rightarrow \bar{q} g$ branchings respectively. However, one finds again that

$$
\xi_{\mathrm{C}}(\gamma) \subseteq \xi(\gamma),
$$

for the three cases, and that all flows belonging to the sets

$$
\xi(\gamma) \backslash \xi_{\mathrm{C}}(\gamma)
$$

do not induce collinear singularities. Therefore, the matrix elements defined in eq. (3.114) have the collinear limits given by eq. (3.120), and can thus be used with the latter and with those of eq. (3.86) for the subtractions of eq. (1.4).

\subsubsection{The $g \rightarrow q \bar{q}$ branching at fixed flows}

As was the case for the other collinear branchings discussed so far, the starting point is the expression of the colour-summed collinear limits (here, eqs. (3.49)-(3.51)), where one writes the scattering amplitudes using their representations in terms of Born colour flows. Therefore:

$$
\begin{aligned}
& \mathcal{M}_{\mathrm{COLL}}^{(2 q ; n+1)}=\sum_{\gamma, \gamma^{\prime} \in \mathcal{F}_{2(q-1) ; n+2}} \mathcal{M}_{\mathrm{COLL}}^{(2 q ; n+1)}\left(\gamma^{\prime}, \gamma\right), \\
& \mathcal{M}_{\mathrm{COLL}}^{(2 q ; n+1)}\left(\gamma^{\prime}, \gamma\right)=\frac{g_{S}^{2}}{k_{-q} \cdot k_{-2 q}}\left\{\hat{P}_{q g}(z) \overline{\mathcal{M}}_{q q}^{(2(q-1) ; n+2)}\left(\gamma^{\prime}, \gamma\right)\right. \\
&\left.+\frac{1}{2} \hat{Q}_{q g^{\star}}(z)\left(\frac{\left\langle k_{-q} k_{-2 q}\right\rangle}{\left[k_{-q} k_{-2 q}\right]} \overline{\mathcal{M}}_{q q,-+}^{(2(q-1) ; n+2)}\left(\gamma^{\prime}, \gamma\right)+\frac{\left[k_{-q} k_{-2 q}\right]}{\left\langle k_{-q} k_{-2 q}\right\rangle} \overline{\mathcal{M}}_{q q,+-}^{(2(q-1) ; n+2)}\left(\gamma^{\prime}, \gamma\right)\right)\right\}
\end{aligned}
$$


where

$$
\begin{aligned}
& \overline{\mathcal{M}}_{q q}^{(2(q-1) ; n+2)}\left(\gamma^{\prime}, \gamma\right)=\left\langle\mathcal{A}^{(2(q-1) ; n+2)}\left(\gamma^{\prime}\right)\left|\sum_{b c} G_{b c}^{\star} G_{b c}\right| \mathcal{A}^{(2(q-1) ; n+2)}(\gamma)\right\rangle \\
& \overline{\mathcal{M}}_{q q, \lambda \bar{\lambda}}^{(2(q-1) ; n+2)}\left(\gamma^{\prime}, \gamma\right)=\left\langle\mathcal{A}_{\lambda}^{(2(q-1) ; n+2)}\left(\gamma^{\prime}\right)\left|\sum_{b c} G_{b c}^{\star} G_{b c}\right| \mathcal{A}_{\bar{\lambda}}^{(2(q-1) ; n+2)}(\gamma)\right\rangle
\end{aligned}
$$

Hence (using the same arguments as in eq. (2.74)), the relevant quantities to compute are the colour vectors:

$$
G_{b c}\left|\mathcal{A}^{(2(q-1) ; n+2)}(\gamma)\right\rangle
$$

(or their bra counterparts), which are the analogues of those in eqs. (3.88)-(3.90) or eq. (2.73). By using the definition of the operator $G_{b c}$ given in eq. (3.53), one sees that the colour part of eq. (3.130) involves the computation of:

$$
\begin{aligned}
\sum_{a_{n+2}} \lambda_{b c}^{a_{n+2}} & \left(\lambda^{a_{\sigma\left(t_{r-1}+1\right)}} \ldots \lambda^{\left.a_{\sigma(\sigma-1}(n+2)-1\right)} \lambda^{a_{n+2}} \lambda^{a_{\sigma\left(\sigma^{-1}(n+2)+1\right)}} \ldots \lambda^{\left.a_{\sigma\left(t_{r}\right)}\right)}\right)_{a_{-r} a_{\mu(-r-q)}}= \\
& \frac{1}{2}\left(\lambda^{a_{\sigma\left(t_{r-1}+1\right)}} \ldots \lambda^{a_{\sigma\left(\sigma^{-1}(n+2)-1\right)}}\right)_{a_{-r} c}\left(\lambda^{a_{\sigma\left(\sigma^{-1}(n+2)+1\right)}} \ldots \lambda^{a_{\sigma\left(t_{r}\right)}}\right)_{b a_{\mu(-r-q)}} \\
& -\frac{1}{2 N} \delta_{b c}\left(\lambda^{a_{\sigma\left(t_{r-1}+1\right)}} \ldots \lambda^{a_{\sigma\left(\sigma^{-1}(n+2)-1\right)}} \lambda^{a_{\sigma\left(\sigma^{-1}(n+2)+1\right)}} \ldots \lambda^{\left.a_{\sigma\left(t_{r}\right)}\right)}\right)_{a_{-r} a_{\mu(-r-q)}}
\end{aligned}
$$

where, as the notation suggests, $r$ is the colour line to which gluon $n+2$ belongs, and the r.h.s. of eq. (3.131) has been computed using eq. (A.5). Therefore, for any Born flow

$$
\gamma \in \mathcal{F}_{2(q-1) ; n+2}, \quad \gamma=\bigcup_{p=1}^{q-1} \gamma_{p}
$$

eq. (3.131) suggests to define two operators as follows:

$$
n+2 \in \gamma_{r}, \quad J \gamma=\left(\bigcup_{\substack{p=1 \\ p \neq r}}^{q-1} \gamma_{p}\right) \bigcup\left(J \gamma_{r}\right), \quad K \gamma=\left(\bigcup_{\substack{p=1 \\ p \neq r}}^{q-1} \gamma_{p}\right) \bigcup\left(K \gamma_{r}\right),
$$

where by construction $J \gamma, K \gamma \in \mathcal{F}_{2 q ; n+1}$, and

$$
\begin{aligned}
J \gamma_{r}= & \left(-r ; \sigma\left(t_{r-1}+1\right), \ldots \sigma\left(\sigma^{-1}(n+2)-1\right) ;-2 q\right) \bigcup \\
& \left(-q ; \sigma\left(\sigma^{-1}(n+2)+1\right), \ldots \sigma\left(t_{r}\right) ; \mu(-r-q)\right), \\
K \gamma_{r}= & \left(-r ; \sigma\left(t_{r-1}+1\right), \ldots \sigma\left(\sigma^{-1}(n+2)-1\right),\right. \\
& \left.\sigma\left(\sigma^{-1}(n+2)+1\right), \ldots \sigma\left(t_{r}\right) ; \mu(-r-q)\right) \bigcup(-q ;-2 q) .
\end{aligned}
$$

In other words, the operator $J$ splits in two the colour line to which gluon $n+2$ belongs. The gluons to the left of gluon $n+2$ now belong to a colour line in which the colour of the quark of the original line forms an antenna with the anticolour of antiquark $-2 q$ (that 
emerges from the $g \rightarrow q \bar{q}$ branching), whereas the gluons to the right of gluon $n+2$ now belong to a colour line in which the colour of quark $-q$ (that emerges from the $g \rightarrow q \bar{q}$ branching) forms an antenna with the anticolour of the antiquark of the original line. On the other hand, operator $K$ simply removes gluon $n+2$ from the original colour line, and creates the colour line that connects $-q$ with $-2 q$; the latter line does not contain any gluons. With the definitions above, one arrives at:

$$
\sum_{b c} G_{b c}\left|\mathcal{A}^{(2(q-1) ; n+2)}(\gamma)\right\rangle=\frac{1}{2}\left|\mathcal{A}_{\mathrm{EXT}}^{(2(q-1) ; n+2)}(J \gamma, \gamma)\right\rangle-\frac{1}{2}\left|\mathcal{A}_{\mathrm{EXT}}^{(2(q-1) ; n+2)}(K \gamma, \gamma)\right\rangle,
$$

where the definition of eq. (3.77) has been extended:

$$
\begin{aligned}
\left|\mathcal{A}_{\mathrm{EXT}}^{(2(q-1) ; n+2)}(\Gamma, \gamma)\right\rangle= & \sum_{\left\{a_{i}\right\}_{i=-2 q}^{n+1}} \Lambda\left(\left\{a_{i}\right\}, \Gamma\right) \widehat{\mathcal{A}}^{(2(q-1) ; n+2)}(\gamma)\left|a_{-2 q}, \ldots a_{n+1}\right\rangle, \\
& \text { for any } \Gamma \in \mathcal{F}_{2 q ; n+1}, \quad \gamma \in \mathcal{F}_{2(q-1) ; n+2} .
\end{aligned}
$$

Note that the factor $1 / N$ that appears in the second term on the r.h.s. of eq. (3.131) is indeed contained in the second term on the r.h.s. of eq. (3.136) owing to the definition of $K$, eq. (3.135). In fact, the latter equation shows that $K \gamma$ features a colour line $(-q ;-2 q)$ which is also a flavour line, and therefore:

$$
\rho(K \gamma)=\rho(\gamma)+1
$$

At this points, one plugs eq. (3.136) into eqs. (3.128) and (3.129). Then, the sums over Born flows in eq. (3.126) can be turned into sums over real flows using the same technique as was employed in eq. (2.80). To be definite, I consider the case of R-flows, that of L-flows being identical. One has

$$
\begin{aligned}
\sum_{\gamma \in \mathcal{F}_{2(q-1) ; n+2}}(f(J \gamma, \gamma)+g(K \gamma, \gamma)) & = \\
& \sum_{\gamma \in \mathcal{F}_{2(q-1) ; n+2}} \sum_{\Gamma \in \mathcal{F}_{2 q ; n+1}}(\delta(\Gamma, J \gamma) f(J \gamma, \gamma)+\delta(\Gamma, K \gamma) g(K \gamma, \gamma)),
\end{aligned}
$$

where I have used the identities:

$$
\begin{aligned}
& 1=\sum_{\Gamma \in \mathcal{F}_{2 q ; n+1}} \delta(\Gamma, J \gamma), \\
& 1=\sum_{\Gamma \in \mathcal{F}_{2 q ; n+1}} \delta(\Gamma, K \gamma),
\end{aligned}
$$

which hold because, for a given $\gamma$, the flows $J \gamma$ and $K \gamma$ exist and are unique. The $\delta$ functions can then be used to get rid of the sum over $\gamma$, which requires fixing $\Gamma$, and solving:

$$
\begin{aligned}
& \Gamma=J \gamma, \\
& \Gamma=K \gamma,
\end{aligned}
$$


for $\gamma$ (these equations are the analogues of eq. (2.82) or eq. (3.94)). I start by considering eq. (3.143). As one can deduce from eq. (3.134), eq. (3.143) has a solution if and only if $\Gamma$ belongs to a subset $\mathcal{F}_{2 q ; n+1}^{(J)}$ of the set of real flows, defined as follows:

$$
\Gamma \equiv(\sigma, \mu, t) \in \mathcal{F}_{2 q ; n+1}^{(J)} \subseteq \mathcal{F}_{2 q ; n+1} \quad \Longleftrightarrow \quad \mu(-2 q) \neq-2 q .
$$

From eq. (3.134), it is also obvious that when such a solution exists, it is also unique, and I shall denote it by $\gamma=J^{-1} \Gamma$. Therefore:

$$
\sum_{\gamma \in \mathcal{F}_{2(q-1) ; n+2}} f(J \gamma, \gamma) \equiv \sum_{\gamma \in \mathcal{F}_{2(q-1) ; n+2}} \sum_{\Gamma \in \mathcal{F}_{2 q ; n+1}} \delta(\Gamma, J \gamma) f(J \gamma, \gamma)=\sum_{\Gamma \in \mathcal{F}_{2 q ; n+1}^{(J)}} f\left(\Gamma, J^{-1} \Gamma\right) .
$$

Let me now turn to eq. (3.144) which, as can be seen from eq. (3.135), has a solution if and only if $\Gamma$ belongs to a subset $\mathcal{F}_{2 q ; n+1}^{(K)}$ of the set of real flows, defined as follows:

$$
\Gamma \equiv(\sigma, \mu, t) \in \mathcal{F}_{2 q ; n+1}^{(K)} \subseteq \mathcal{F}_{2 q ; n+1} \quad \Longleftrightarrow \quad \mu(-2 q)=-2 q \text { and } t_{q-1}=t_{q} \equiv n+1 .
$$

At variance with the case of the operator $J$, however, the solution of eq. (3.144) is not unique. In fact, the operator $K$ just removes gluon $n+2$ from the first $q-1$ colour lines, replacing it with the colour line $(-q ;-2 q)$. Hence, the Born flows in which all quarks and gluons have the same relative positions, up to that of gluon $n+2$, will result in the same real flow $K \gamma$. It is easy to convince oneself that this happens exactly $(n+q)$ times. I shall therefore define the multi-valued inverse of the operator $K$, which will give the solutions to eq. (3.144), as follows:

$$
K_{i}^{-1} \Gamma \in \mathcal{F}_{2(q-1) ; n+2} \quad \text { for any } \quad 1 \leq i \leq n+q, \quad \Gamma \in \mathcal{F}_{2 q ; n+1}^{(K)} .
$$

The explicit form of $K_{i}^{-1} \Gamma$ can be worked out from eq. (3.135); it corresponds to inserting gluon $n+2$ in all possible ways in $\Gamma$, except in the colour line $(-q,-2 q)$, which is removed. ${ }^{5}$ Putting all this together, one obtains:

$$
\sum_{\gamma \in \mathcal{F}_{2(q-1) ; n+2}} g(K \gamma, \gamma) \equiv \sum_{\gamma \in \mathcal{F}_{2(q-1) ; n+2}} \sum_{\Gamma \in \mathcal{F}_{2 q ; n+1}} \delta(\Gamma, K \gamma) g(K \gamma, \gamma)=\sum_{\Gamma \in \mathcal{F}_{2 q ; n+1}^{(K)}} \sum_{i=1}^{n+q} g\left(\Gamma, K_{i}^{-1} \Gamma\right)
$$

Counting arguments can again be given as a consistency check of the results obtained here. The number of elements in the sets of flows relevant to the derivation above are:

$$
\begin{aligned}
\#\left(\mathcal{F}_{2(q-1) ; n+2}\right) & =(q-1) !(n+2) ! \mathcal{N}(n+2, q-1), \\
\#\left(\mathcal{F}_{2 q ; n+1}^{(J)}\right) & =(q-1)(q-1) !(n+1) ! \mathcal{N}(n+1, q), \\
\#\left(\mathcal{F}_{2 q ; n+1}^{(K)}\right) & =(q-1) !(n+1) ! \mathcal{N}(n+1, q-1),
\end{aligned}
$$

with $\mathcal{N}$ given in eq. (3.101). By direct computation, one shows that

$$
\begin{aligned}
& \#\left(\mathcal{F}_{2(q-1) ; n+2}\right)=\#\left(\mathcal{F}_{2 q ; n+1}^{(J)}\right), \\
& \#\left(\mathcal{F}_{2(q-1) ; n+2}\right)=\#\left(\mathcal{F}_{2 q ; n+1}^{(K)}\right)(n+q) .
\end{aligned}
$$

\footnotetext{
${ }^{5}$ This implies that $K^{-1}$ could be written in terms of the operators $I_{+}$defined for gluon $n+2$, if need be.
} 
Hence, the same number of terms appear on the two sides of eqs. (3.146) and (3.149). Equations (3.126)-(3.129) can now be expressed in terms of real flows:

$$
\begin{gathered}
\mathcal{M}_{\mathrm{COLL}}^{(2 q ; n+1)}=\sum_{\Gamma, \Gamma^{\prime} \in \mathcal{F}_{2 q ; n+1}^{(J)} \cup \mathcal{F}_{2 q ; n+1}^{(K)}} \mathcal{M}_{\mathrm{COLL}}^{(2 q ; n+1)}\left(\Gamma^{\prime}, \Gamma\right), \\
\mathcal{M}_{\mathrm{COLL}}^{(2 q ; n+1)}\left(\Gamma^{\prime}, \Gamma\right)=\frac{g_{S}^{2}}{4 k_{-q} \cdot k_{-2 q}}\left\{\hat{P}_{q g}(z) \mathcal{M}_{\mathrm{EXT}, \mathrm{AB}}^{(2(q-1) ; n+2)}\left(\Gamma^{\prime}, \Gamma\right)\right. \\
\left.+\frac{1}{2} \hat{Q}_{q g^{\star}}(z)\left(\frac{\left\langle k_{-q} k_{-2 q}\right\rangle}{\left[k_{-q} k_{-2 q}\right]} \mathcal{M}_{\mathrm{EXT}, \mathrm{AB},-+}^{(2(q-1) ; n+2)}\left(\Gamma^{\prime}, \Gamma\right)+\frac{\left[k_{-q} k_{-2 q}\right]}{\left\langle k_{-q} k_{-2 q}\right\rangle} \mathcal{M}_{\mathrm{EXT,AB},+-}^{(2(q-1) ; n+2)}\left(\Gamma^{\prime}, \Gamma\right)\right)\right\},
\end{gathered}
$$

where, in eq. (3.156):

$$
A, B \in\{J, K\}, \quad \Gamma^{\prime} \in \mathcal{F}_{2 q ; n+1}^{(A)}, \quad \Gamma \in \mathcal{F}_{2 q ; n+1}^{(B)} .
$$

The reduced matrix elements squared are defined as follows:

$$
\begin{aligned}
& \mathcal{M}_{\mathrm{EXT}, \mathrm{JJ}}^{(2(q-1) ; n+2)}\left(\Gamma^{\prime}, \Gamma\right)=\left\langle\mathcal{A}_{\mathrm{EXT}}^{(2(q-1) ; n+2)}\left(\Gamma^{\prime}, J^{-1} \Gamma^{\prime}\right) \mid \mathcal{A}_{\mathrm{EXT}}^{(2(q-1) ; n+2)}\left(\Gamma, J^{-1} \Gamma\right)\right\rangle, \\
& \mathcal{M}_{\mathrm{EXT}, \mathrm{JK}}^{(2(q-1) ; n+2)}\left(\Gamma^{\prime}, \Gamma\right)=-\sum_{j=1}^{n+q}\left\langle\mathcal{A}_{\mathrm{EXT}}^{(2(q-1) ; n+2)}\left(\Gamma^{\prime}, J^{-1} \Gamma^{\prime}\right) \mid \mathcal{A}_{\mathrm{EXT}}^{(2(q-1) ; n+2)}\left(\Gamma, K_{j}^{-1} \Gamma\right)\right\rangle, \\
& \mathcal{M}_{\mathrm{EXT}, \mathrm{KJ}}^{(2(q-1) ; n+2)}\left(\Gamma^{\prime}, \Gamma\right)=-\sum_{i=1}^{n+q}\left\langle\mathcal{A}_{\mathrm{EXT}}^{(2(q-1) ; n+2)}\left(\Gamma^{\prime}, K_{i}^{-1} \Gamma^{\prime}\right) \mid \mathcal{A}_{\mathrm{EXT}}^{(2(q-1) ; n+2)}\left(\Gamma, J^{-1} \Gamma\right)\right\rangle, \\
& \mathcal{M}_{\mathrm{EXT}, \mathrm{KK}}^{(2(q-1) ; n+2)}\left(\Gamma^{\prime}, \Gamma\right)=\sum_{i=1}^{n+q} \sum_{j=1}^{n+q}\left\langle\mathcal{A}_{\mathrm{EXT}}^{(2(q-1) ; n+2)}\left(\Gamma^{\prime}, K_{i}^{-1} \Gamma^{\prime}\right) \mid \mathcal{A}_{\mathrm{EXT}}^{(2(q-1) ; n+2)}\left(\Gamma, K_{j}^{-1} \Gamma\right)\right\rangle,
\end{aligned}
$$

and similarly for the azimuthal terms $\mathcal{M}_{\mathrm{EXT}, \mathrm{AB}, \lambda \bar{\lambda}}^{(2(-1) ; n+2)}$, which as usual only require the use of amplitudes at given polarizations. The matrix elements of eqs. (3.158)-(3.161) all feature the same colour structure, as is implied by eq. (3.137). This becomes evident if one expresses them in terms of scalar quantities:

$$
\begin{aligned}
\mathcal{M}_{\mathrm{EXT}, \mathrm{JJ}}^{(2(q-1) ; n+2)}\left(\Gamma^{\prime}, \Gamma\right)= & \widehat{\mathcal{A}}^{(2(q-1) ; n+2)}\left(J^{-1} \Gamma^{\prime}\right)^{\star} C\left(\Gamma^{\prime}, \Gamma\right) \widehat{\mathcal{A}}^{(2(q-1) ; n+2)}\left(J^{-1} \Gamma\right), \\
\mathcal{M}_{\mathrm{EXT}, \mathrm{JK}}^{(2(q-1) ; n+2)}\left(\Gamma^{\prime}, \Gamma\right)= & -\widehat{\mathcal{A}}^{(2(q-1) ; n+2)}\left(J^{-1} \Gamma^{\prime}\right)^{\star} C\left(\Gamma^{\prime}, \Gamma\right)\left(\sum_{j=1}^{n+q} \widehat{\mathcal{A}}^{(2(q-1) ; n+2)}\left(K_{j}^{-1} \Gamma\right)\right), \\
\mathcal{M}_{\mathrm{EXT}, \mathrm{KJ}}^{(2(q-1) ; n+2)}\left(\Gamma^{\prime}, \Gamma\right)= & -\left(\sum_{i=1}^{n+q} \widehat{\mathcal{A}}^{(2(q-1) ; n+2)}\left(K_{i}^{-1} \Gamma^{\prime}\right)^{\star}\right) C\left(\Gamma^{\prime}, \Gamma\right) \widehat{\mathcal{A}}^{(2(q-1) ; n+2)}\left(J^{-1} \Gamma\right), \\
\mathcal{M}_{\mathrm{EXT}, \mathrm{KK}}^{(2(q-1) ; n+2)}\left(\Gamma^{\prime}, \Gamma\right)= & \left(\sum_{i=1}^{n+q} \widehat{\mathcal{A}}^{(2(q-1) ; n+2)}\left(K_{i}^{-1} \Gamma^{\prime}\right)^{\star}\right) C\left(\Gamma^{\prime}, \Gamma\right) \\
& \times\left(\sum_{j=1}^{n+q} \widehat{\mathcal{A}}^{(2(q-1) ; n+2)}\left(K_{j}^{-1} \Gamma\right)\right)
\end{aligned}
$$


As is implied by eq. (3.155), a closed flow $\left(\Gamma^{\prime}, \Gamma\right)$ will not induce a singularity if either its L-flow or its R-flow (or both) does not belong to the set $\mathcal{F}_{2 q ; n+1}^{(J)} \cup \mathcal{F}_{2 q ; n+1}^{(K)}$.

Collinear singularities due to $g \rightarrow q \bar{q}$ branchings can obviously occur when the underlying Born amplitude is a pure-gluon one, which corresponds to $q=1$. The notation used so far is not suited to describe such a case, the Born amplitudes having been written as quark-gluon ones. It is however not difficult to extend what was done before to $q=1$, since one can formally understand

$$
\left|\mathcal{A}^{(2(q-1) ; n+2)}(\gamma)\right\rangle \stackrel{q=1}{\longrightarrow}\left|\mathcal{A}^{(0 ; n+2)}(\gamma)\right\rangle \equiv\left|\mathcal{A}^{(n+2)}(\sigma)\right\rangle,
$$

with $\sigma$ being the only non-trivial part of the flow $\gamma$ in this case, namely a permutation of gluon labels (one must also understand that the permutations are restricted to be noncyclic only, when $q=1$ is considered). Equation (3.130) is then still relevant, but its colour part is not given by eq. (3.131), but rather by:

$$
\begin{aligned}
& \sum_{a_{n+2}} \lambda_{b c}^{a_{n+2}} \operatorname{Tr}\left(\lambda^{a_{\sigma(1)}} \ldots \lambda^{a_{\sigma\left(\sigma^{-1}(n+2)-1\right)}} \lambda^{a_{n+2}} \lambda^{a_{\sigma\left(\sigma^{-1}(n+2)+1\right)}} \ldots \lambda^{a_{\sigma(n+2)}}\right)= \\
& \frac{1}{2}\left(\lambda^{a_{\sigma\left(\sigma^{-1}(n+2)+1\right)}} \ldots \lambda^{a_{\sigma(n+2)}} \lambda^{a_{\sigma(1)}} \ldots \lambda^{a_{\sigma\left(\sigma^{-1}(n+2)-1\right)}}\right)_{b c} \\
& -\frac{1}{2 N} \delta_{b c} \operatorname{Tr}\left(\lambda^{a_{\sigma(1)}} \ldots \lambda^{a_{\sigma\left(\sigma^{-1}(n+2)-1\right)}} \lambda^{a_{\sigma\left(\sigma^{-1}(n+2)+1\right)}} \ldots \lambda^{a_{\sigma(n+2)}}\right) .
\end{aligned}
$$

The second term on the r.h.s. of eq. (3.167) does not belong to the set of real flows; hence, it must give a contribution to the final result equal to zero. That this is indeed the case can be proved by direct computation. One starts by extending the definitions of the operators $J$ and $K$ to the case $q=1$ :

$$
\begin{gathered}
J \gamma=\left(-q ; \sigma\left(\sigma^{-1}(n+2)+1\right), \ldots \sigma(n+2),\right. \\
\left.\sigma(1), \ldots \sigma\left(\sigma^{-1}(n+2)-1\right) ;-2 q\right), \\
K \gamma=\left(\sigma(1), \ldots \sigma\left(\sigma^{-1}(n+2)-1\right),\right. \\
\left.\sigma\left(\sigma^{-1}(n+2)+1\right), \ldots \sigma(n+2)\right) \bigcup(-q ;-2 q) .
\end{gathered}
$$

With these, one proceeds exactly as done before. The only difference is that now

$$
\mathcal{F}_{2 q ; n+1}^{(J)}=\mathcal{F}_{2 q ; n+1}
$$

As far as $\mathcal{F}_{2 q ; n+1}^{(K)}$ is concerned, one simply defines it as the set of flows that have the same form as the one in eq. (3.169). By doing that, ones arrives again at eqs. (3.155)-(3.161). However, the following reduced matrix elements are identically equal to zero:

$$
\mathcal{M}_{\mathrm{EXT}, \mathrm{JK}}^{(0 ; n+2)}\left(\Gamma^{\prime}, \Gamma\right)=\mathcal{M}_{\mathrm{EXT}, \mathrm{KJ}}^{(0 ; n+2)}\left(\Gamma^{\prime}, \Gamma\right)=\mathcal{M}_{\mathrm{EXT}, \mathrm{KK}}^{(0 ; n+2)}\left(\Gamma^{\prime}, \Gamma\right)=0,
$$

since they contain one or both of the linear combinations

$$
\sum_{i=1}^{n+1} \mathcal{A}^{(0 ; n+2)}\left(K_{i}^{-1} \Gamma^{\prime}\right)=\sum_{j=1}^{n+1} \mathcal{A}^{(0 ; n+2)}\left(K_{j}^{-1} \Gamma\right)=0,
$$


which are equal to zero owing to the dual Ward identity [1]. This proves the fact that the second term on the r.h.s. of eq. (3.167) indeed does not contribute to the final result. The collinear limit for the $g \rightarrow q \bar{q}$ branching in the case of a pure-gluon Born is therefore still given by eqs. (3.155) and (3.156), with the condition that $\mathcal{F}_{2 q ; n+1}^{(K)}$ be equal to the empty set, and taking eq. (3.171) into account.

In analogy with what was done previously, I should now discuss the case of the $g \rightarrow q \bar{q}$ branching at fixed Born flows. However, it does not appear to be possible to formulate it in a gauge-invariant manner. This is due to the fact that the operator $K$ does not have a single-valued inverse. If one must keep the Born flows $K_{i}^{-1} \Gamma$ separate, rather than summing them as done in eqs. (3.159)-(3.161), one must also "split" the contributions to the real flow $\Gamma$ into $(n+q)$ components, in order for each of them to have a limit proportional to $\Lambda\left(K_{i}^{-1} \Gamma\right)$. This may be possible by considering individual Feynman diagrams, at the price of violating gauge invariance. It does not seem justified to do so since, contrary to the cases of $g \rightarrow g g$ and $q \rightarrow q g$ branchings, the Altarelli-Parisi kernel associated with the $g \rightarrow q \bar{q}$ branching is $\mathcal{O}\left(N^{0}\right)$, which implies that for this branching the two formulations at fixed Born or real flows are strictly equivalent from the colour point of view. The above discussion does not apply to the case $q=1$, i.e. when one has a pure-gluon Born since, as was shown before, when $q=1$ the $K$ operator does not contribute to the result. But it is obvious that in such a case fixing Born flows is identical to fixing real flows.

\section{Summary of subtraction formulae}

Since the detailed derivations carried out in sections 2 and 3 can obscure the final results, in this section I collect the references to the formulae needed for the implementation of FKS subtraction at fixed colour configurations or flows. For the colour-summed real-emission matrix element squared $\mathcal{M}$ and its limit $\mathcal{M}_{\mathrm{L}}$ (be it soft, collinear, or soft-collinear):

$$
\mathcal{M} \stackrel{\mathrm{L}}{\longrightarrow} \mathcal{M}_{\mathrm{L}}, \quad \mathrm{L}=\mathrm{SOFT}, \mathrm{COLL}, \mathrm{SC},
$$

I have used the decompositions:

$$
\mathcal{M}=\sum_{g} \mathcal{M}(g), \quad \mathcal{M}_{\mathrm{L}}=\sum_{g} \mathcal{M}_{\mathrm{L}}(g)
$$

with

$$
\mathcal{M}(g) \stackrel{\mathrm{L}}{\longrightarrow} \mathcal{M}_{\mathrm{L}}(g) \quad \forall g .
$$

I have considered three cases, which I list here using the notations relevant to gluon and to quark-gluon amplitudes respectively. As discussed in the text, the soft-collinear limit $\mathcal{M}_{\mathrm{SC}}(g)$ can be trivially obtained by computing either the collinear limit of $\mathcal{M}_{\text {SOFT }}(g)$, or the soft limit of $\mathcal{M}_{\mathrm{COLL}}(g)$; hence, it will not be considered in what follows.

- Fixed colour configurations:

$$
g=\left(a_{1}, \ldots a_{n+1}\right), \quad g=\left(a_{-2 q}, \ldots a_{n+1}\right) .
$$


The matrix elements $\mathcal{M}(g)$ are defined in eq. (2.48) for gluon amplitudes, and in eq. (3.56) for quark-gluon amplitudes.

Soft limits $\mathcal{M}_{\text {SОFт }}(g)$. For gluon amplitudes: eqs. (2.51) and (2.53). For quark-gluon amplitudes: eqs. (3.57) and (3.59).

Collinear limits $\mathcal{M}_{\mathrm{COLL}}(g)$. For gluon amplitudes, eqs. (2.55), (2.58), and (2.59). For quark-gluon amplitudes, and $g \rightarrow g g, q \rightarrow q g$, or $\bar{q} \rightarrow \bar{q} g$ branchings: eqs. (3.61), (3.64), and (3.65). For quark-gluon amplitudes, and $g \rightarrow q \bar{q}$ branchings: eqs. (3.67), (3.70), and (3.71).

- Fixed real flows:

$$
g=\left(\Sigma^{\prime}, \Sigma\right), \quad g=\left(\Gamma^{\prime}, \Gamma\right) .
$$

The matrix elements $\mathcal{M}(g)$ are defined in eq. (2.62) for gluon amplitudes, and in eq. (3.74) for quark-gluon amplitudes. The definitions of flows in the two cases are given at the beginning of section 2 and of section 3 respectively.

Soft limits $\mathcal{M}_{\text {SOFт }}(g)$. For gluon amplitudes: eqs. (2.90) and (2.93). For quark-gluon amplitudes: eqs. (3.105) and (3.107).

Collinear limits $\mathcal{M}_{\mathrm{COLL}}(g)$. For gluon amplitudes, eqs. (2.110), (2.93), and (2.112). For quark-gluon amplitudes, and $g \rightarrow g g, q \rightarrow q g$, or $\bar{q} \rightarrow \bar{q} g$ branchings: eqs. (3.109), (3.107), and (3.112). For quark-gluon amplitudes, and $g \rightarrow q \bar{q}$ branchings: eqs. (3.156) and (3.162)-(3.165).

- Fixed Born flows:

$$
g=\left(\sigma^{\prime}, \sigma\right), \quad g=\left(\gamma^{\prime}, \gamma\right) .
$$

The matrix elements $\mathcal{M}(g)$ relevant to this case are linear combinations of those defined at fixed real flows. They are given in eqs. (2.117) and (2.114) for gluon amplitudes, and in eqs. (3.114) and (3.116) for quark-gluon amplitudes.

Soft limits $\mathcal{M}_{\text {SOFT }}(g)$. For gluon amplitudes: eqs. (2.69) and (2.119). For quarkgluon amplitudes: eqs. (3.86) and (3.115).

Collinear limits $\mathcal{M}_{\mathrm{COLL}}(g)$. For gluon amplitudes, eqs. (2.120), (2.20), and (2.121). For quark-gluon amplitudes, and $g \rightarrow g g, q \rightarrow q g$, or $\bar{q} \rightarrow \bar{q} g$ branchings: eqs. (3.120)(3.122). For quark-gluon amplitudes, and $g \rightarrow q \bar{q}$ branchings: this case cannot be treated at fixed Born flows.

\section{Born-like contributions}

I have so far discussed the case of the real-emission matrix elements and of their local subtraction terms. NLO cross sections also receive other contributions, which have a Born-like kinematics. These contributions are due to the Born proper, to the one-loop corrections, and to the analytically-integrated subtraction terms. In this section, I shall discuss the treatment of the latter when one fixes the colour configurations or the colour flows.

In FKS one defines two types of Born-like contributions, which arise from the analytical integration of either the soft or the collinear counterterms. The one of soft origin reads as 
follows, up to overall trivial factors (see e.g. ref. [11]):

$$
\sum_{k, l=-2 q}^{n} \mathcal{E}_{k l} \mathcal{M}_{k l}^{(2 q ; n)}
$$

where I have used the notation of the quark-gluon amplitudes case for generality. Equation (5.1) coincides with eq. (3.33), except for the fact that the eikonal factors $[k, l]$ in the latter equation have been replaced in eq. (5.1) by the finite parts of their integrals, denoted by $\mathcal{E}_{k l}$. The eikonals do not play any role in the manipulations carried out in sections 2 and 3. Hence, one can just use the results for the soft limits of the matrix elements presented before and summarized in section 4 , and simply replace $[k, l]$ with $\mathcal{E}_{k l}$ there.

The Born-like contribution of collinear origin is proportional to the Born matrix element squared:

$$
\mathcal{Q} \mathcal{M}^{(2 q ; n)}
$$

where

$$
\begin{aligned}
\mathcal{Q}=\sum_{k=-2 q}^{n} & {\left[\gamma^{\prime}\left(\mathcal{I}_{k}\right)-\log \frac{s \delta_{O}}{2 Q^{2}}\left(\gamma\left(\mathcal{I}_{k}\right)-2 C\left(\mathcal{I}_{k}\right) \log \frac{2 E_{k}}{\xi_{\text {cut }} \sqrt{s}}\right)\right.} \\
& \left.+2 C\left(\mathcal{I}_{k}\right)\left(\log ^{2} \frac{2 E_{k}}{\sqrt{s}}-\log ^{2} \xi_{\text {cut }}\right)-2 \gamma\left(\mathcal{I}_{k}\right) \log \frac{2 E_{k}}{\sqrt{s}}\right] \delta_{0 m_{k}},
\end{aligned}
$$

and

$$
\begin{aligned}
\gamma(g) & =\frac{11}{6} C_{A}-\frac{2}{3} T_{F} N_{f}, \\
\gamma(q) & =\frac{3}{2} C_{F} \\
\gamma^{\prime}(g) & =\left(\frac{67}{9}-\frac{2 \pi^{2}}{3}\right) C_{A}-\frac{23}{9} T_{F} N_{f}, \\
\gamma^{\prime}(q) & =\left(\frac{13}{2}-\frac{2 \pi^{2}}{3}\right) C_{F} .
\end{aligned}
$$

The definitions of the various quantities that appear in eq. (5.3) are irrelevant here; the interested reader may find them in ref. [11]. What matters is the definition of the colour factors, given in eqs. (5.4)-(5.7). These basically arise from the integration of the AltarelliParisi kernels: $P_{q q}$ for $\gamma(q)$ and $\gamma^{\prime}(q)$ ( $q \rightarrow q g$ and $\bar{q} \rightarrow \bar{q} g$ branchings); $P_{g g}$ plus $P_{q g}$ for $\gamma(g)$ and $\gamma^{\prime}(g)\left(g \rightarrow g g\right.$ and $g \rightarrow q \bar{q}$ branchings). The sum of $P_{g g}$ and $P_{q g}$ is necessary because $\gamma(g)$ and $\gamma^{\prime}(g)$ are inclusive properties of gluons, and therefore all possible branchings must be taken into account. However, these can still be told apart in eqs. (5.4) and (5.6), thanks to the different colour factors $\left(C_{A}\right.$ versus $\left.T_{F}\right)$. This implies that, in eq. (5.3), one can unambiguously associate the various terms with either a $q \rightarrow q g$ or a $\bar{q} \rightarrow \bar{q} g$ branching $\left(C_{F}\right.$ terms), or a $g \rightarrow g g$ branching $\left(C_{A}\right.$ terms), or a $g \rightarrow q \bar{q}$ branching $\left(T_{F}\right.$ terms). For each of these branching types, one can then repeat what was done in sections 2 and 3 in the case of collinear limits. This implies starting from re-writing eq. (5.2) as follows:

$$
\mathcal{Q} \mathcal{M}^{(2 q ; n)}=\sum_{k=-2 q}^{-1} C_{F}(\ldots) \mathcal{M}^{(2 q ; n)}+\sum_{k=1}^{n} C_{A}(\ldots) \mathcal{M}^{(2 q ; n)}+\sum_{k=1}^{n} T_{F}(\ldots) \mathcal{M}^{(2 q ; n)}
$$


where the terms in brackets can be easily worked out from eqs. (5.3)-(5.7). Their forms are irrelevant here, except for the fact that they do not contain any colour factors. At this point, one exploits eq. (3.39) in the first two terms of eq. (5.8), to replace $\mathcal{M}^{(2 q ; n)}$ with $\overline{\mathcal{M}}_{k}^{(2 q ; n)}$ defined in eq. (3.43) (with $s \rightarrow k$ there). Analogously, one exploits eq. (3.54) in the last term of eq. (5.8), to replace $\mathcal{M}^{(2 q ; n)}$ with $\overline{\mathcal{M}}_{q q}^{(2(q-1) ; n+2)}$ defined in eq. (3.50). After these replacements, the matrix elements in eq. (5.8) are in the same form as those that appear in the colour-summed expressions of the collinear limits. Hence, the same manipulations as in sections 2 and 3 can be carried out here; the final results can be directly obtained from the expressions of the collinear limits at fixed colour configurations or real flows, by simply replacing the Altarelli-Parisi kernels and prefactors there with the expressions in round brackets that appear in eq. (5.8). Note that this procedure must not be carried out if one is interested in fixing Born flows: the original expression, eq. (5.2), is already suited to that, the only change being the formal replacement:

$$
\mathcal{M}^{(2 q ; n)} \longrightarrow \mathcal{M}^{(2 q ; n)}\left(\gamma^{\prime}, \gamma\right)
$$

\section{Discussion}

Processes with large particle multiplicities have a colour algebra so involved that its direct computation is impossible, and one must use alternative methods, such as Monte Carlo (MC) ones. While it is common to sum over colour configurations with MC techniques, there is no reason of principle that prevents one from doing an $\mathrm{MC}$ sum over colour flows such a strategy is indeed being considered in the new version of MadGraph/MadEvent [31]. At the tree level, matrix elements at fixed colour configurations (e.g. eq. (2.18)) have the advantage over those at fixed flows (e.g. eq. (2.20)) of being positive definite, while the latter are in general complex numbers. However, this advantage is lost beyond the leading order in perturbation theory, since subtractions such as those of eq. (1.4) will always be involved, thus implying results of either sign. Also, in an actual computation one will consider the sum $\mathcal{M}\left(\sigma^{\prime}, \sigma\right)+\mathcal{M}\left(\sigma, \sigma^{\prime}\right)$, which is a real number, since physical observables must not depend on the distinction between L- and R-flows. Furthermore, at any order in perturbation theory, including tree level, matrix elements at fixed colour flows have an immediate interpretation in terms of large- $N$ expansion, which is rather indirect at fixed colour configurations. In particular, it is not necessary to explicitly compute the colourflow matrix of eq. (2.21) (or eq. (3.76)) to determine the largest possible power of $N$ in any of its elements - it is sufficient to count the number of colour loops determined by the closed flow $\left(\sigma^{\prime}, \sigma\right)$ (or $\left(\gamma^{\prime}, \gamma\right)$ ), which is basically instantaneous if performed by a computer. This fact not only paves the way to a systematic organization of the computation in terms of increasing powers of $1 / N$, but also suggests a way to save computing time, since terms with large $1 / N$ powers may be computed with a relatively low statistics.

The results presented in this paper allow one to follow either of the strategies discussed above in the context of the computation of NLO observables with FKS subtraction. The approach that uses fixed colour configurations bases its efficiency on the fast calculation of colour-dressed amplitudes, at both the real-emission and Born levels. It should be 
stressed that, for a given real matrix element at fixed colours, such e.g. that of eq. (2.48) for gluon amplitudes, one must compute several Born-level colour-dressed amplitudes see eq. (2.53) for the soft limit, and eqs. (2.58) and (2.59) for the collinear limit. It is clear that, for the sake of numerical stability, the sums over $b_{k}^{\prime}$ and $b_{l}$ in eq. (2.53), and those over $b_{n}^{\prime}$ and $b_{n}$ in eqs. (2.58) and (2.59), must be performed exactly, and not with MC methods. Furthermore, in the case of the soft limits the sums over $k$ and $l$ that appear in eq. (2.51) must also be taken into account. The bottom line is that, if one performs these sums blindly, a non-negligible complexity creeps back into the game. For a fast computation of the counterterms, then, an algorithm is essential that pre-determines which colour configurations are associated with tree-level amplitudes that are equal to zero. It is probably also important to compute the colour matrices $\mathcal{Q}_{\mathcal{I}_{k} \mathcal{I}_{l}}$ and $\mathcal{G}$ once and for all, since many of their elements will be equal to zero, an information which can be effectively used to improve the efficiency of the algorithm. These issues are beyond the scope of this paper, and will not be discussed any further.

Turning to the fixed-flow approach, the general arguments related to the $1 / N$ expansion apply to both the fixed-real and the fixed-Born flow cases. I also stress that, in order to derive the formulae for the fixed-flow subtraction terms, I have started from the known limiting behaviours of the squared matrix elements, the same that I have used in the case of fixed colour configurations. In other words, I did not employ the limits of colour-ordered amplitudes. While the latter may have provided a more straightforward derivation for fixed real flows, the approach followed here allows the treatment of the three schemes considered (fixed colour configurations, Born flows, and real flows) in a common language. The fact that the results I have arrived at for the matrix element limits at fixed real flows can also be derived using the limits of dual amplitudes as a starting point constitutes a partial cross check of the procedure adopted in this paper.

When fixing the Born flows, the building blocks of the counterterms are the same ones as those used in the automatic implementation of the FKS subtraction achieved by MadFKS [11]. Furthermore, in this scheme the simultaneous integration of the real-emission and of the Born-like and Born contributions can be performed in exactly the same way as in ref. [11], where it is discussed in detail. On the other hand, from the point of view of computational complexity, for any pair of Born-level dual amplitudes that determine the counterterms, one needs to evaluate $n^{2}$ (in the case of gluon amplitudes - see eqs. (2.117) and $(2.114)$ ), or $(n+q)^{2}$ (in the case of quark-gluon amplitudes - see eqs. (3.114) and (3.116)), real-emission dual amplitudes. This has an obvious physical interpretation: the sets of real flows $\zeta(\sigma)$ and $\xi(\gamma)$ effectively achieve a block decomposition of the colourflow matrices $C\left(\Sigma^{\prime}, \Sigma\right)$ and $C\left(\Gamma^{\prime}, \Gamma\right)$ respectively. The choice of a Born-level closed flow corresponds to choosing one of these blocks; the relevant colour algebra at the real-emission level is then that of the block so determined. In other words, when performing an MC sum over Born flows, each seed is associated with a block in the matrix of real colour flows; within this block, the colour algebra is performed exactly, i.e. without using MC methods.

Let me finally discuss the case of fixed real flows. This scheme has the nice property that the colour-flow matrix can be factored out of the subtraction procedure. In other words, all of the four terms in the linear combination of eq. (1.4) are proportional either to 
$C\left(\Sigma^{\prime}, \Sigma\right)$ (for gluon amplitudes) or to $C\left(\Gamma^{\prime}, \Gamma\right)$ (for quark-gluon amplitudes). This implies that colour never enters into the definition of subtraction terms if not in a trivial way, which does not happen (owing to the definition of colour-linked Born's) in the other formulations of the FKS subtraction given here, let alone in the colour-summed one. A pleasant implication of this fact is the disappearance of the colour-linked Born's from the soft subtraction terms (see eqs. (2.90) and (3.105)). This, which is nothing but the colourless nature of the subtraction procedure, together with the possibility of defining the soft and collinear kinematics so as the underlying Born kinematics coincide, implies the following equation (which I write for quark-gluon amplitudes, for greater generality, and without considering the case of the $g \rightarrow q \bar{q}$ branching, which I shall discuss later):

$$
\begin{aligned}
\mathcal{S}_{i j} \widehat{\mathcal{A}}^{(2 q ; n+1)}\left(\Gamma^{\prime}\right)^{\star} & \widehat{\mathcal{A}}^{(2 q ; n+1)}(\Gamma) \longrightarrow \\
& \mathcal{K}_{i j}\left(\Gamma^{\prime}, \Gamma\right) \widehat{\mathcal{A}}^{(2 q ; n)}\left(\Gamma_{\ell}^{\prime}\right)^{\star} \widehat{\mathcal{A}}^{(2 q ; n)}\left(\Gamma_{\ell}\right) \\
& +\mathcal{K}_{i j-+}^{(\varphi)}\left(\Gamma^{\prime}, \Gamma\right) \widehat{\mathcal{A}}_{-}^{(2 q ; n)}\left(\Gamma_{\ell}^{\prime}\right)^{\star} \widehat{\mathcal{A}}_{+}^{(2 q ; n)}\left(\Gamma_{\ell}\right) \\
& +\mathcal{K}_{i j+-}^{(\varphi)}\left(\Gamma^{\prime}, \Gamma\right) \widehat{\mathcal{A}}_{+}^{(2 q ; n)}\left(\Gamma_{\ell}^{\prime}\right)^{\star} \widehat{\mathcal{A}}_{-}^{(2 q ; n)}\left(\Gamma_{\ell}\right),
\end{aligned}
$$

where the arrow denotes the soft, collinear, or soft-collinear limit. The kernel $\mathcal{K}_{i j}$ is thus able to describe the singular behaviour of the interference between dual amplitudes in these three limits. The only contribution to the divergences that cannot be included in $\mathcal{K}_{i j}$ is the azimuthal-dependent part of the collinear limit, for which the kernels $\mathcal{K}_{i j \lambda \bar{\lambda}}^{(\varphi)}$ need be introduced, owing to the different structure of the factorized Born-level dual amplitudes (the helicity of the branching parton is not summed over in the last two terms on the r.h.s. of eq. (6.1)). ${ }^{6}$ As is known, the azimuthal terms vanish upon integration, but must nevertheless be included in the subtraction, in order to have a local cancellation of divergences in all phase-space points, and not only at the integrated level. Needless to say, in eq. (6.1) and in what follows all terms of collinear origin are present only if parton $j$ is not a massive quark. The explicit form of the kernels that appear in eq. (6.1) can be worked out from eqs. (3.105) and (3.109). One obtains:

$$
\begin{aligned}
\mathcal{K}_{i j}\left(\Gamma^{\prime}, \Gamma\right)= & -\left.g_{S}^{2}\left(\sum_{\alpha=-1,1} \sum_{\beta=-1,1} \alpha \beta\left[\Gamma^{\prime}\left(\Gamma^{\prime-1}(i) \oplus \alpha\right), \Gamma\left(\Gamma^{-1}(i) \oplus \beta\right)\right]\right) \mathcal{S}_{i j}\right|_{\mathrm{SOFT}} \\
& +\left.\frac{g_{S}^{2}}{k_{i} \cdot k_{j}} \delta\left(\Gamma^{\prime}, \Gamma\right) \hat{P}_{\mathcal{I}_{j} \mathcal{I}_{j}}^{(+)}(z)\right|_{\mathrm{COLL}}, \\
\mathcal{K}_{i j-+}^{(\varphi)}\left(\Gamma^{\prime}, \Gamma\right)= & \left.\frac{g_{S}^{2}}{2 k_{i} \cdot k_{j}} \delta\left(\Gamma^{\prime}, \Gamma\right) \hat{Q}_{\mathcal{I}_{j} \mathcal{I}_{j}^{\star}}(z) \frac{\left\langle k_{i} k_{j}\right\rangle}{\left[k_{i} k_{j}\right]}\right|_{\mathrm{COLL}}, \\
\mathcal{K}_{i j+-}^{(\varphi)}\left(\Gamma^{\prime}, \Gamma\right)= & \left.\frac{g_{S}^{2}}{2 k_{i} \cdot k_{j}} \delta\left(\Gamma^{\prime}, \Gamma\right) \hat{Q}_{\mathcal{I}_{j} \mathcal{I}_{j}^{\star}}(z) \frac{\left[k_{i} k_{j}\right]}{\left\langle k_{i} k_{j}\right\rangle}\right|_{\mathrm{COLL}} .
\end{aligned}
$$

Several comments are in order here. First of all, being clear that the results obtained in section 3 are valid for any partons, I have relabeled $n+1$ and $s$ as $i$ and $j$ respectively, consistently with the role of $\mathcal{S}_{i j}$ in eq. (6.1); it is however understood that parton $i$ is

\footnotetext{
${ }^{6}$ Alternatively, one can define in a straightforward manner a unique kernel, as a tensor in the space of the helicities of the branching parton. This is what is done e.g. in dipole subtraction.
} 
a gluon. The SOFT and COLL tags in eqs. (6.2)-(6.4) imply that the four momenta used to compute the corresponding quantities are those relevant to the soft and collinear configurations respectively. In the antenna or dipole formulations, the analogues of these configurations are explicitly defined by means of invariants. In FKS, one does not need an explicit definition; any maps induced by the real-emission phase-space parametrization will do, provided that the latter satisfies the condition that the underlying Born kinematics obtained by taking the soft and collinear limits coincide. ${ }^{7}$ The exactly collinear configurations used in the computation of the second term on the r.h.s. of eq. (6.2), and in eqs. (6.3) and (6.4), is the reason why no $\mathcal{S}$ function appear there - in such a limit, $\mathcal{S}_{i j}=1$ [13]. The $(+)$ index attached to the Altarelli-Parisi kernel in the second term on the r.h.s. of eq. (6.2) implies that such a kernel must be understood as being subtracted by means of a plus prescription. It is easy to convince oneself (see e.g. eq. (2.29)) that this is sufficient to take into account the soft-collinear limit (last term in eq. (1.4)). Although in principle the same should be done for the azimuthal kernels of eqs. (6.3) and (6.4), in practice this is not necessary, owing to the fact that $\hat{Q}_{a b^{\star}}(z)$ vanish in the soft limit. I finally point out that eqs. (6.1)-(6.4) are valid also in the case of $g \rightarrow q \bar{q}$ branching, with only changes in notation. However, because of the vanishing of the soft limit in this case, the final result would be identical to that already presented in eqs. (3.156) and (3.162)-(3.165).

I have already shown in section 5 that Born-like contributions to the NLO cross section can also be cast in the form of terms with fixed real flows. As far as the Born proper is concerned, in order to integrate it simultaneously with the other terms one can use the same trick as that used to transform eq. (5.2) into eq. (5.8) - this is possible since the Born can be split into several components associated with $\mathcal{S}_{i j}$ functions, as explained in ref. [11], thus effectively defining Born contributions at fixed real flows. Another possibility is that of exploiting the block structure of the real colour-flow matrix discussed above: for a given real flow $\Gamma$, there exists a Born flow $\gamma$ such that $\Gamma \in \xi(\gamma)$. In this way, for any random choice of $\Gamma$ in the MC sum over real flows, one also performs an MC sum over Born flows (which is unbiased, at least as long as $\Gamma$ is chosen flat).

I have presented the results in the form of unphysical $0 \rightarrow m$ processes for ease of notation. It is clear that they have the same structure, colour-wise, when two of the final-state particles are crossed into the initial state. It should be kept in mind that for physical $2 \rightarrow$ $m-2$ processes there is contribution to the NLO cross section which is essentially a Born-like one, except for the fact that it features an additional integration variable. This contribution, called "degenerate $(n+1)$-body" in ref. [11], has the same form as the collinear limits discussed at length in this paper, and can therefore be manipulated in exactly the same way.

Antenna and dipole subtractions. It seems appropriate to conclude this discussion by stressing the connections between FKS, antenna, and dipole subtractions, which are best uncovered when a fixed-real-flow scheme is adopted in the former. Firstly, given the fact that antenna subtraction is formulated in terms of dual amplitudes or of quantities closely

\footnotetext{
${ }^{7}$ As was mentioned at the end of section 2.1, this condition is necessary for eq. (6.1) to hold. It is however not mandatory in general for FKS subtraction, but has been found to be convenient from the numerical point of view.
} 
related to those, it is natural that FKS at fixed real flows use the same matrix elements limits as in antenna (see e.g. eq. (3.105) and its counterpart obtained in ref. [32], in the context of a process-specific computation). As was mentioned before, this is reassuring, since the factorization formulae one starts from do not coincide in the two approaches. From the kinematical point of view, however, FKS and antenna are still fairly different. In particular, in FKS one of the two radiators that define an antenna is not needed (the other radiator may be identified with the FKS sister), and this is reflected in the different choices made for the phase-space parametrizations (which in FKS do not depend on colour connections).

Secondly, the methods used here to manipulate the colour-linked Born's (which for example lead one from eqs. (3.85)-(3.87) to eqs. (3.104)-(3.106)) can also be used to carry out the same operations on dipoles. More specifically, the structure of the colour kernel of a dipole $\mathcal{D}_{i j, k}$ is (in the notation of this paper) $\sum_{b} Q^{b}(i j) Q^{b}(k)$, up to an overall Casimir. The most involved case is when either $i$ or $j$ (or both) is a gluon — be it $i$ just to fix the notation. Then, one can identify $i j$ and $k$ of the colour kernel above with $l$ and $k$ in eq. (3.86), $i$ with the FKS parton, and proceed as done in this paper. ${ }^{8}$ In this way, a linear combination of dipoles will emerge, analogue to that featuring the eikonal factors in eq. (6.2):

$$
\sum_{\alpha=-1,1} \sum_{\beta=-1,1} \alpha \beta \mathcal{D}_{i j, k}\left(j \rightarrow \Gamma^{\prime}\left(\Gamma^{\prime^{-1}}(i) \oplus \alpha\right), k \rightarrow \Gamma\left(\Gamma^{-1}(i) \oplus \beta\right)\right) .
$$

As was the case for the colour-linked Born's, all the dipoles in eq. (6.5) will factorize the same colour-flow matrix element $C\left(\Gamma^{\prime}, \Gamma\right)$. However, different dipoles will factorize dual amplitudes (which sandwich helicity-dependent kernels) computed with different kinematics, and therefore the analogue of eq. (6.1) cannot be written in this case. This problem can be avoided by using variants of the dipole formalism that curb the proliferation of reduced kinematics (see e.g. refs $[33,34]$ ).

The similarities among the various subtraction formalisms need not be surprising; as was shown in ref. [35], all of them must have the same underlying structure, and differences arise when choices are made for the projections that map resolved kinematic configurations onto unresolved ones, and for the definitions of subtraction terms away from the zeromeasure soft and collinear regions. Colours somehow blur the picture, which becomes clear again if one works in schemes such as the fixed-real-flow one (which may not be unique in this respect).

\section{Conclusions}

The results of this paper will allow the implementation of the FKS formalism in a colourfriendly way, since the elementary ingredients of the subtraction procedure can be defined at fixed colour configurations or colour flows. This is a necessary condition in order to

\footnotetext{
${ }^{8}$ When $j$ is a gluon, $\mathcal{D}_{i j, k}$ also subtracts the soft singularities associated with $j$, whereas in FKS the soft singularities of the FKS sister are damped. In order to simplify the discussion, I assume here that such a damping is performed also in dipoles, thus a-symmetrizing the roles of $i$ and $j$. Many other options are obviously possible.
} 
be able to perform the colour algebra with Monte Carlo methods, and thus to tackle the computation of large-multiplicity processes. The formulation of the subtraction at fixed colour flows can be used to organize the calculations as a systematic expansion in $1 / N$, since all results given here are exact to all orders in $N$. When the flows are fixed at the real-emission level, the colour and Lorentz structures completely decouple, and this allows one to define colourless kernels that can simultaneously describe the soft, collinear, or softcollinear behaviour of the matrix element squared. In this scheme, one sees more clearly the connections between the FKS subtraction method and the antenna and dipoles ones, which I have briefly discussed.

\section{Acknowledgments}

I am indebted to Fabio Maltoni for the countless discussions we have had on this matter (and on many others). I thank Michelangelo Mangano for patiently listening to, and answering, my many questions. Correspondence with Thomas Gehrmann and Rikkert Frederix is also gratefully acknowledged. This manuscript has benefited from comments by Fabio, Michelangelo, and Rikkert.

\section{A Conventions for colour matrices}

Contrary to the usual conventions, in this paper I normalize the Gell-Mann matrices in the same way as the $\mathrm{SU}(N)$ generators. Hence:

$$
\begin{aligned}
{\left[\lambda^{a}, \lambda^{b}\right] } & =i \sum_{c=1}^{N^{2}-1} f^{a b c} \lambda^{c}, \\
\operatorname{Tr}\left(\lambda^{a} \lambda^{b}\right) & =T_{F} \delta^{a b}, \\
\sum_{a=1}^{N^{2}-1}\left(\lambda^{a} \lambda^{a}\right)_{i j} & =C_{F} \delta_{i j}, \\
\sum_{a, b=1}^{N^{2}-1} f^{a b c} f^{a b d} & =C_{A} \delta^{c d}, \\
\sum_{a=1}^{N^{2}-1} \lambda_{i j}^{a} \lambda_{k l}^{a} & =\frac{1}{2}\left(\delta_{i l} \delta_{j k}-\frac{1}{N} \delta_{i j} \delta_{k l}\right),
\end{aligned}
$$

with the usual colour factors

$$
\begin{aligned}
T_{F} & =\frac{1}{2}, \\
C_{F} & =\frac{N^{2}-1}{2 N}, \\
C_{A} & =N .
\end{aligned}
$$




\section{B Colour operators, flows, and colour conservation}

As shown in sections 2.1 and 3.1 for gluon and quark-gluon amplitudes respectively, the consistency between the soft and collinear limits of the matrix elements follows from the colour-conservation identities of eqs. (2.45) and (3.47). These identities are in turn a consequence of expressing the scattering amplitudes in terms of dual amplitudes, as done in eqs. (2.4) and (3.14), as I shall show in this appendix.

An important by-product of this proof is the fact that the colour operators $Q^{b}$, while defined in a natural way in the spaces of colour configurations as in eqs. (2.33)-(2.34) and (3.36)-(3.38), can also be easily interpreted in terms of flows. I shall therefore introduce operators acting on flows that have an explicit correspondence with $Q^{b}$, and which will be instrumental in deriving a formulation of the FKS subtraction alternative to that at fixed colour configurations.

\section{B.1 Gluon amplitudes}

Since eq. (2.45) is an identity in the colour space of $n+1$ gluons, it can be rewritten as follows

$$
X \equiv \sum_{k=1}^{n}\left\langle a_{1}, \ldots a_{n+1}\left|Q^{b}(k)\right| \mathcal{A}^{(n)}\right\rangle=0 \quad \forall\left\{a_{i}\right\}_{i=1}^{n+1} .
$$

Note that eq. (B.1) is equivalent to saying that colour conservation applies to $(n+1)$-gluon amplitudes at fixed colour configurations. Using eq. (2.13), one can rewrite

$$
\begin{aligned}
X & =\sum_{\sigma \in P_{n}^{\prime}} X_{\sigma} \\
X_{\sigma} & =\sum_{k=1}^{n}\left\langle a_{1}, \ldots a_{n+1}\left|Q^{b}(k)\right| \mathcal{A}^{(n)}(\sigma)\right\rangle .
\end{aligned}
$$

Therefore, if one can prove that $X_{\sigma}=0$ for an arbitrary $\sigma$, eq. (B.1) will follow (the converse is obviously not true, and thus $X_{\sigma}=0$ is a stronger conditions than $X=0$ ). By using eqs. (2.11), (2.33), and (2.34), one obtains

$$
X_{\sigma}=Y_{\sigma} \widehat{\mathcal{A}}^{(n)}(\sigma),
$$

where

$$
\begin{aligned}
Y_{\sigma} & =\sum_{k=1}^{n} \sum_{b_{k}}\left(T^{a_{n+1}}\right)_{a_{k} b_{k}} \operatorname{Tr}\left(\lambda^{a_{\sigma(1)}} \ldots \lambda^{a_{\sigma\left(\sigma^{-1}(k)-1\right)}} \lambda^{b_{k}} \lambda^{a_{\sigma\left(\sigma^{-1}(k)+1\right)}} \ldots \lambda^{a_{\sigma(n)}}\right) \\
& =\sum_{k=1}^{n} \operatorname{Tr}\left(\lambda^{a_{\sigma(1)}} \ldots \lambda^{a_{\sigma\left(\sigma^{-1}(k)-1\right)}}\left[\lambda^{a_{k}}, \lambda^{a_{n+1}}\right] \lambda^{a_{\sigma\left(\sigma^{-1}(k)+1\right)}} \ldots \lambda^{a_{\sigma(n)}}\right) .
\end{aligned}
$$

Hence, in order to prove that $X_{\sigma}=0$, one must prove that $Y_{\sigma}=0$. I start from observing that eq. (B.6) is a linear combination of traces obtained by inserting $\lambda^{a_{n+1}}$ into the traces 
that appear in $\left|\mathcal{A}^{(n)}(\sigma)\right\rangle$. Such insertions can be conveniently represented in terms of flows. This can be done by defining the following operators:

$$
\begin{aligned}
I_{+}(i) \sigma & \equiv I_{+}(i)(\sigma(1), \ldots \sigma(n))=(\sigma(1), \ldots \sigma(i), n+1, \ldots \sigma(n)), \\
I_{-}(i) \sigma & \equiv I_{-}(i)(\sigma(1), \ldots \sigma(n))=(\sigma(1), \ldots n+1, \sigma(i), \ldots \sigma(n)) .
\end{aligned}
$$

In other words, $I_{+}(i)\left(I_{-}(i)\right)$ inserts the number $n+1$ into the list defined by $\sigma$ after (before) the $i^{\text {th }}$ member of the list. One can now rewrite eq. (B.6) as follows, using the shorthand notation of eq. (2.5):

$$
Y_{\sigma}=\sum_{k=1}^{n}\left\{\Lambda\left(I_{+}\left(\sigma^{-1}(k)\right) \sigma\right)-\Lambda\left(I_{-}\left(\sigma^{-1}(k)\right) \sigma\right)\right\}
$$

A comparison of eq. (B.9) with eq. (B.3) shows the relationship between the $Q^{b}$ and the $I_{ \pm}$operators:

$$
Q^{b} \longleftrightarrow I_{+}-I_{-} .
$$

In keeping with the fact that $Q^{b}$ creates a colour state associated with gluon $n+1$, the operators $I_{ \pm}$transform a $n$-gluon flow into an $(n+1)$-gluon flow. A technical difference between the operators $Q^{b}$ and $I_{ \pm}$is that the argument of the former is a particle label, whereas the arguments of the latter are the positions in the list of particles that defines the flow. This is justified by the fact that when manipulating flows it is more convenient to deal with positions rather than particle labels - singularity configurations are due to particles that are adjacent in a flow. It is obvious that summing over particle labels is equivalent to summing over positions in a flow. Hence, from eq. (B.9):

$$
Y_{\sigma}=\sum_{i=1}^{n}\left\{\Lambda\left(I_{+}(i) \sigma\right)-\Lambda\left(I_{-}(i) \sigma\right)\right\}
$$

Furthermore

$$
\begin{aligned}
\sum_{i=1}^{n} \Lambda\left(I_{-}(i) \sigma\right) & =\Lambda\left(I_{-}(1) \sigma\right)+\sum_{i=2}^{n} \Lambda\left(I_{-}(i) \sigma\right) \\
& =\Lambda\left(I_{+}(n) \sigma\right)+\sum_{i=2}^{n} \Lambda\left(I_{+}(i-1) \sigma\right) \\
& =\Lambda\left(I_{+}(n) \sigma\right)+\sum_{i=1}^{n-1} \Lambda\left(I_{+}(i) \sigma\right) \\
& =\sum_{i=1}^{n} \Lambda\left(I_{+}(i) \sigma\right)
\end{aligned}
$$

where the various manipulations follow from the invariance of the trace under cyclic permutations, and from the property

$$
I_{-}(i) \sigma=I_{+}(i-1) \sigma, \quad 2 \leq i \leq n,
$$


which trivially follows from the definitions of $I_{ \pm}(i)$. By replacing eq. (B.12) into eq. (B.11) one proves that $Y_{\sigma}=0$. As discussed at the beginning of this section, this not only proves eq. (2.45), but also that

$$
\sum_{k=1}^{n} Q^{b}(k)\left|\mathcal{A}^{(n)}(\sigma)\right\rangle=0 .
$$

In other words, colour conservation also holds at fixed Born flows; this is far from surprising from the physics viewpoint.

\section{B.2 Quark-gluon amplitudes}

In the case of quark-gluon amplitudes, the proof of eq. (3.47) proceeds through proving the analogue of eq. (B.1), i.e.:

$$
X \equiv \sum_{k=-2 q}^{n}\left\langle a_{-2 q}, \ldots a_{n+1}\left|Q^{b}(k)\right| \mathcal{A}^{(2 q ; n)}\right\rangle=0 \quad \forall\left\{a_{i}\right\}_{i=-2 q}^{n+1} .
$$

Following what is done in the case of gluon amplitudes, one writes

$$
\begin{aligned}
X & =\sum_{\gamma \in \mathcal{F}_{2 q ; n}} X_{\gamma}, \\
X_{\gamma} & =\sum_{k=-2 q}^{n}\left\langle a_{-2 q}, \ldots a_{n+1}\left|Q^{b}(k)\right| \mathcal{A}^{(2 q ; n)}(\gamma)\right\rangle .
\end{aligned}
$$

As was done before, I shall show that the condition $X_{\gamma}=0$ holds, thereby proving eq. (B.15) as well. Using eq. (3.29), one gets

$$
X_{\gamma}=Y_{\gamma} \widehat{\mathcal{A}}^{(2 q ; n)}(\gamma),
$$

where now

$$
Y_{\gamma}=\sum_{\left\{c_{i}\right\}_{i=-2 q}^{n}} \Lambda\left(\left\{c_{i}\right\}, \gamma\right) \sum_{k=-2 q}^{n}\left\langle a_{-2 q}, \ldots a_{n+1}\left|Q^{b}(k)\right| c_{-2 q}, \ldots c_{n}\right\rangle .
$$

Owing to the factorized form of the colour structure, eq. (3.15), one can associate a colour structure with each colour line:

$$
\begin{aligned}
\Lambda\left(\left\{a_{i}\right\}, \gamma\right) & =N^{-\rho(\gamma)} \prod_{p=1}^{q} \Lambda\left(\left\{a_{i}\right\}, \gamma_{p}\right), \\
\Lambda\left(\left\{a_{i}\right\}, \gamma_{p}\right) & =\left(\lambda^{a_{\sigma\left(t_{p-1}+1\right)}} \ldots \lambda^{a_{\sigma\left(t_{p}\right)}}\right)_{a_{-p} a_{\mu(-p-q)}} .
\end{aligned}
$$

Using the definition of the operator $Q^{b}(k)$, eq. (B.19) then becomes:

$$
\begin{aligned}
Y_{\gamma} & =N^{-\rho(\gamma)} \sum_{p=1}^{q}\left(\prod_{\substack{r=1 \\
r \neq p}}^{q} \Lambda\left(\left\{a_{i}\right\}, \gamma_{r}\right)\right) Y_{\gamma, p}, \\
Y_{\gamma, p} & =\sum_{k \in \gamma_{p}} \sum_{c_{k}}\left(Q^{a_{n+1}}(k)\right)_{a_{k} c_{k}} \Lambda\left(\left\{a_{i}\right\}_{i \neq k}, c_{k}, \gamma_{p}\right) .
\end{aligned}
$$


Equation (B.23) states formally that the action of the operators $Q^{b}$ onto the scattering amplitude can be conveniently rewritten colour line per colour line. It also implies that in order to prove $X_{\gamma}=0$ a sufficient condition is that of proving $Y_{\gamma, p}=0$ for all $p$, which is what I set out to do now. First, by using eqs. (2.34), (3.37) and (3.38), one obtains:

$$
\begin{aligned}
& \sum_{c_{k}}\left(Q^{a_{n+1}}(k)\right)_{a_{k} c_{k}} \Lambda\left(\left\{a_{i}\right\}_{i \neq k}, c_{k}, \gamma_{p}\right)= \\
& \left(\lambda^{a_{\sigma\left(t_{p-1}+1\right)}} \ldots \lambda^{a_{\sigma\left(\sigma^{-1}(k)-1\right)}}\left[\lambda^{a_{k}}, \lambda^{a_{n+1}}\right] \lambda^{a_{\sigma\left(\sigma^{-1}(k)+1\right)}} \ldots \lambda^{a_{\sigma\left(t_{p}\right)}}\right)_{a_{-p} a_{\mu(-p-q)}}, \\
& \sum_{c_{k}}\left(Q^{a_{n+1}}(k)\right)_{a_{k} c_{k}} \Lambda\left(\left\{a_{i}\right\}_{i \neq k}, c_{k}, \gamma_{p}\right)=\left(\lambda^{a_{n+1}} \lambda^{a_{\sigma\left(t_{p-1}+1\right)}} \ldots \lambda^{a_{\sigma\left(t_{p}\right)}}\right)_{a_{-p} a_{\mu(-p-q)}},
\end{aligned}
$$

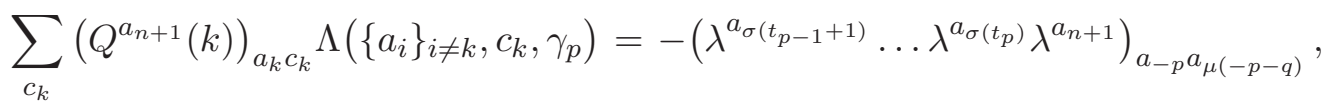

for the cases when $k$ is a gluon, a quark, or an antiquark respectively. Note that in the latter two cases the requirement that $k \in \gamma_{p}$ implies $k=-p$ and $k=\mu(-p-q)$ respectively. Precisely as in the case of gluon amplitudes, eqs. (B.24)-(B.26) suggest the use of operators acting on flows. For consistency with what was done before, the arguments of these operators will have to be the positions of the particles that appear in flows. My conventions for such positions are the following. The colour line of eq. (3.26)

$$
\gamma_{p}=\left(-p ; \sigma\left(t_{p-1}+1\right), \ldots \sigma\left(t_{p}\right) ; \mu(-p-q)\right)
$$

corresponds to positions:

$$
\left(-p ; t_{p-1}+1, \ldots t_{p} ;-p-q\right) .
$$

This implies that $-p$ is adjacent to $t_{p-1}+1$, and that $-p-q$ is adjacent to $t_{p}$. It is therefore convenient to define the operations $\oplus 1$ and $\ominus 1$, that will serve to move across the list in eq. (B.28):

$$
\begin{aligned}
i \oplus 1 & = \begin{cases}t_{p-1}+1 & i=-p, \\
i+1 & t_{p-1}+1 \leq i<t_{p}, \\
-p-q & i=t_{p},\end{cases} \\
i \ominus 1 & = \begin{cases}-p & i=t_{p-1}+1, \\
i-1 & t_{p-1}+1<i \leq t_{p}, \\
t_{p} & i=-p-q,\end{cases} \\
i \oplus 1 \ominus 1 & =i \ominus 1 \oplus 1=i, \\
i \oplus-1 & =i \ominus 1 .
\end{aligned}
$$

The definitions in eqs. (B.29) and (B.30) imply that one can move continuously across a given colour line, but cannot pass continuously from line $\gamma_{p}$ to line $\gamma_{p \pm 1}$. This is consistent with the physical interpretation of colour lines, which from the colour viewpoint are disconnected from each other, and with the fact that when studying singularities at fixed flows the emphasis is on adjacent particles. Equations (B.28)-(B.30) are meant to hold for 
all colour lines $\gamma_{p}$ belonging to a given flow $\gamma$. Note that in the case of eq. (3.23), i.e. when there are no gluons on colour line $\gamma_{p},-p$ and $-p-q$ are contiguous, and therefore

$$
\left.\begin{array}{rl}
(-p) \oplus 1 & =-p-q \\
(-p-q) \ominus 1 & =-p
\end{array}\right\} \text { if } t_{p-1}=t_{p}
$$

Finally, I shall denote by $\gamma^{-1}(k)$ the position of particle $k$ in flow $\gamma$ (exactly as $\sigma^{-1}(k)$ denotes the position of gluon $k$ in flow $\sigma$ ). Thus, eqs. (B.24)-(B.26) can be rewritten as follows:

$$
\begin{aligned}
& \sum_{c_{k}}\left(Q^{a_{n+1}}(k)\right)_{a_{k} c_{k}} \Lambda\left(\left\{a_{i}\right\}_{i \neq k}, c_{k}, \gamma_{p}\right)= \\
& \Lambda\left(\left\{a_{i}\right\}, I_{+}\left(\gamma^{-1}(k)\right) \gamma_{p}\right)-\Lambda\left(\left\{a_{i}\right\}, I_{-}\left(\gamma^{-1}(k)\right) \gamma_{p}\right), \\
& \sum_{c_{k}}\left(Q^{a_{n+1}}(k)\right)_{a_{k} c_{k}} \Lambda\left(\left\{a_{i}\right\}_{i \neq k}, c_{k}, \gamma_{p}\right)=\Lambda\left(\left\{a_{i}\right\}, I_{+}\left(\gamma^{-1}(k)\right) \gamma_{p}\right), \\
& \sum_{c_{k}}\left(Q^{a_{n+1}}(k)\right)_{a_{k} c_{k}} \Lambda\left(\left\{a_{i}\right\}_{i \neq k}, c_{k}, \gamma_{p}\right)=-\Lambda\left(\left\{a_{i}\right\}, I_{-}\left(\gamma^{-1}(k)\right) \gamma_{p}\right) .
\end{aligned}
$$

As in the case of gluon amplitudes, the result of $I_{+}(i)\left(I_{+}(i)\right)$ acting on the list associated with the flow is that of inserting the number $n+1$ after (before) the $i^{\text {th }}$ member of the list. Given the factorized form of the flow, eq. (3.25), the operators $I_{ \pm}(i)$ can be equivalently understood as acting on the colour flow $\gamma$, or on the colour line $\gamma_{p}$ which includes the $i^{\text {th }}$ position. The conventions adopted before imply that:

$$
\begin{array}{llrl}
I_{+}\left(\gamma^{-1}(k)\right) & =I_{+}(-p) & k & =-p, \\
I_{-}\left(\gamma^{-1}(k)\right) & =I_{-}(-p-q) & k & =\mu(-p-q) .
\end{array}
$$

Putting all this together, one obtains:

$$
Y_{\gamma, p}=\sum_{i=t_{p-1}+1 \ominus 1}^{t_{p}} \Lambda\left(\left\{a_{i}\right\}, I_{+}(i) \gamma_{p}\right)-\sum_{i=t_{p-1}+1}^{t_{p} \oplus 1} \Lambda\left(\left\{a_{i}\right\}, I_{-}(i) \gamma_{p}\right) .
$$

By construction, the analogue of eq. (B.13) holds:

$$
I_{-}(i) \gamma_{p}=I_{+}(i \ominus 1) \gamma_{p}, \quad t_{p-1}+1 \leq i \leq t_{p} \oplus 1 .
$$

Hence

$$
\begin{aligned}
\sum_{i=t_{p-1}+1}^{t_{p} \oplus 1} \Lambda\left(\left\{a_{i}\right\}, I_{-}(i) \gamma_{p}\right) & =\sum_{i=t_{p-1}+1}^{t_{p} \oplus 1} \Lambda\left(\left\{a_{i}\right\}, I_{+}(i \ominus 1) \gamma_{p}\right) \\
& =\sum_{i=t_{p-1}+1 \ominus 1}^{t_{p}} \Lambda\left(\left\{a_{i}\right\}, I_{+}(i) \gamma_{p}\right)
\end{aligned}
$$

where in the last equation I relabeled the sum variable $i \rightarrow i \ominus 1$. By plugging eq. (B.42) into eq. (B.39) one gets finally gets $Y_{\gamma, p}=0$. It is easy to convince oneself that the derivation above also applies to the case of a colour line with no gluons attached, since in such a case:

$$
I_{+}(-p) \gamma_{p}=I_{-}(-p-q) \gamma_{p} \quad \text { if } t_{p-1}=t_{p}
$$



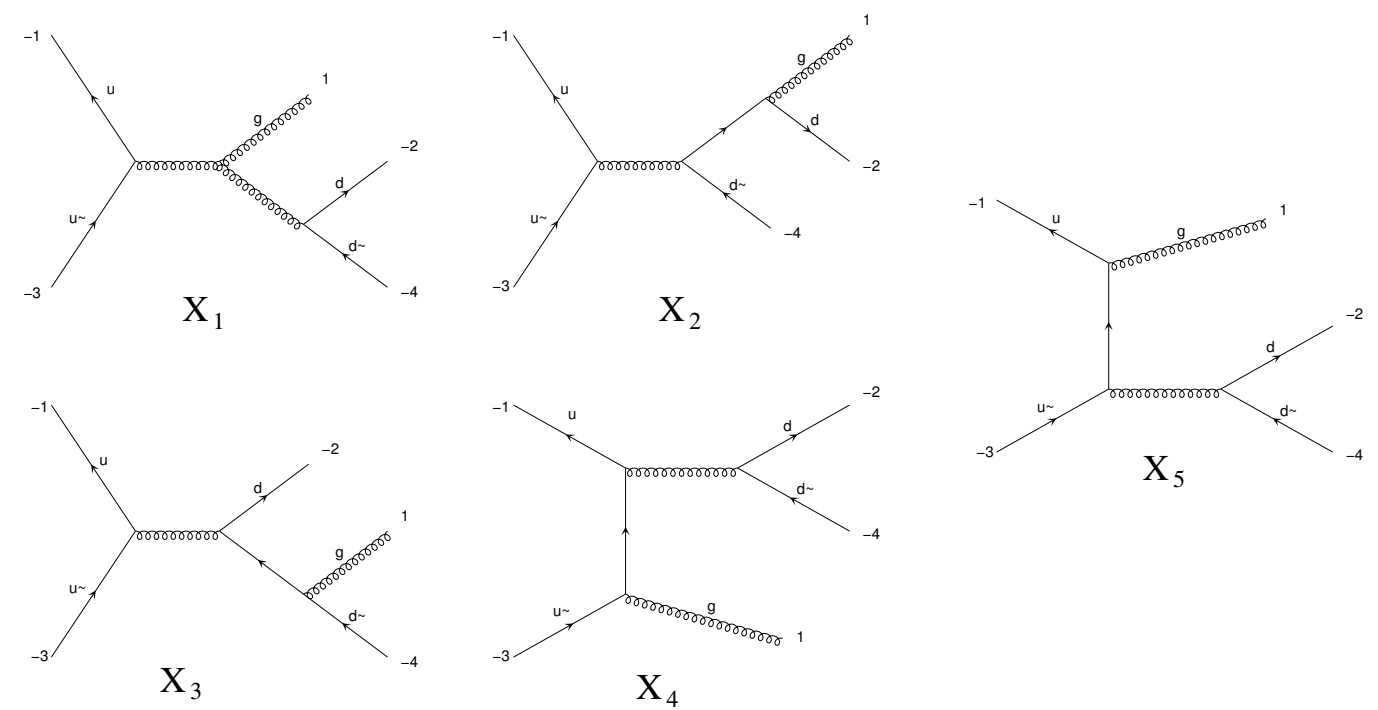

Figure 1. Diagrams that contribute to $0 \rightarrow u \bar{u} d \bar{d} g$.

To take this fact into account, one may perform the following formal replacement:

$$
\sum_{i=t_{p-1}+1 \ominus 1}^{t_{p}} f(i) \longrightarrow\left(1-\delta_{t_{p-1} t_{p}}\right) \sum_{i=t_{p-1}+1 \ominus 1}^{t_{p}} f(i)+\delta_{t_{p-1} t_{p}} \delta_{i(-p)} f(i) .
$$

In practice, the replacement in eq. (B.44) will always be understood. Given that the arguments above apply to any $p$, one has indeed proved eq. (3.47), and also that

$$
\sum_{k=-2 q}^{n} Q^{b}(k)\left|\mathcal{A}^{(n)}(\gamma)\right\rangle=0
$$

analogously to what happens in the case of gluon amplitudes.

\section{A simple example: $0 \rightarrow u \bar{u} d \bar{d} g$}

In this appendix I shall illustrate the practical application of some of the techniques presented in this paper, by considering the real-emission process

$$
0 \rightarrow u(-1) \bar{u}(-3) d(-2) \bar{d}(-4) g(1),
$$

where the parton labelling follows the conventions introduced in section 3. The diagrams that contribute to the process of eq. (C.1) are depicted in figure 1. A straightforward calculation leads one to writing the amplitude, whose general form is given in eq. (3.14), as follows:

$$
\mathcal{X}=\sum_{i=1}^{4} \Lambda\left(\Gamma_{i}\right) \widehat{\mathcal{X}}\left(\Gamma_{i}\right)
$$

where the colour flows are

$$
\begin{array}{ll}
\Gamma_{1}=(-1 ;-4)(-2 ; 1 ;-3), & \Gamma_{2}=(-1 ; 1 ;-4)(-2 ;-3), \\
\Gamma_{3}=(-1 ;-3)(-2 ; 1 ;-4), & \Gamma_{4}=(-1 ; 1 ;-3)(-2 ;-4),
\end{array}
$$


which, according to eq. (3.15), correspond to:

$$
\begin{aligned}
\Lambda\left(\Gamma_{1}\right) & =\delta_{a_{-1} a_{-4}} \lambda_{a_{-2} a_{-3}}^{a_{1}}, & \Lambda\left(\Gamma_{2}\right) & =\delta_{a_{-2} a_{-3}} \lambda_{a_{-1} a_{-4}}^{a_{1}}, \\
\Lambda\left(\Gamma_{3}\right) & =\frac{1}{N} \delta_{a_{-1} a_{-3}} \lambda_{a_{-2} a_{-4}}^{a_{1}}, & \Lambda\left(\Gamma_{4}\right) & =\frac{1}{N} \delta_{a_{-2} a_{-4}} \lambda_{a_{-1} a_{-3}}^{a_{1}},
\end{aligned}
$$

and the dual amplitudes are defined in terms of Feynman diagrams (stripped of colour factors):

$$
\begin{array}{ll}
2 \widehat{\mathcal{X}}\left(\Gamma_{1}\right)=-i X_{1}+X_{2}+X_{5}, & 2 \widehat{\mathcal{X}}\left(\Gamma_{2}\right)=i X_{1}+X_{3}+X_{4}, \\
2 \widehat{\mathcal{X}}\left(\Gamma_{3}\right)=-X_{2}-X_{3}, & 2 \widehat{\mathcal{X}}\left(\Gamma_{4}\right)=-X_{4}-X_{5} .
\end{array}
$$

By direct computation, the colour-flow matrix of eq. (3.76)

$$
C_{i j}^{(X)} \equiv C\left(\Gamma_{i}, \Gamma_{j}\right)
$$

turns out to read:

$$
\begin{aligned}
& C_{11}^{(X)}=C_{22}^{(X)}=L C, \\
& C_{12}^{(X)}=C_{21}^{(X)}=C_{34}^{(X)}=C_{43}^{(X)}=0, \\
& C_{i j}^{(X)}=S C,
\end{aligned}
$$$$
\text { all other } i, j \text {, }
$$

with

$$
L C=\left(N^{3}-N\right) / 2, \quad S C=N / 2-1 /(2 N) .
$$

Therefore, only the closed flows $\left(\Gamma_{1}, \Gamma_{1}\right)$ and $\left(\Gamma_{2}, \Gamma_{2}\right)$ give leading-colour contributions to the cross section, the other closed flows being subleading in colour, and associated with the same power of $N$. In turn, this suggests to define:

$$
p\left(\Gamma^{\prime}, \Gamma\right)=\# \text { colour loops }-\rho\left(\Gamma^{\prime}\right)-\rho(\Gamma),
$$

where the number of colour loops is that identified by the closed flow $\left(\Gamma^{\prime}, \Gamma\right)$, and the function $\rho$ has been defined in eq. (3.16). The larger $p\left(\Gamma^{\prime}, \Gamma\right)$, the more leading in colour the contribution of the closed flow $\left(\Gamma^{\prime}, \Gamma\right)$ to the cross section. In the example I am considering in this appendix, one has $p=3$ for the closed flows in eq. (C.10), and $p=1$ for those in eq. (C.12). I stress that the computation of $p$ can be carried out without calculating traces of Gell-Mann matrices, since both the number of colour loops and the value of $\rho$ can be obtained directly from the colour flows as given in eqs. (3.25) and (3.26).

I now turn to discussing the calculation of the soft and of the $g\|d, d\| \bar{d}$ collinear limits of the process of eq. (C.1), which are representative of all possible singular configurations. Since I shall consider analytical results here, the treatment at fixed colour configurations is not particularly illuminating, and I shall rather concentrate on that at fixed (Born or real) colour flows. The following underlying Born processes will be relevant for the study of the limits:

$$
\begin{aligned}
& 0 \rightarrow u(-1) \bar{u}(-3) d(-2) \bar{d}(-4), \\
& 0 \rightarrow u(-1) \bar{u}(-3) g(1) g(2)
\end{aligned}
$$



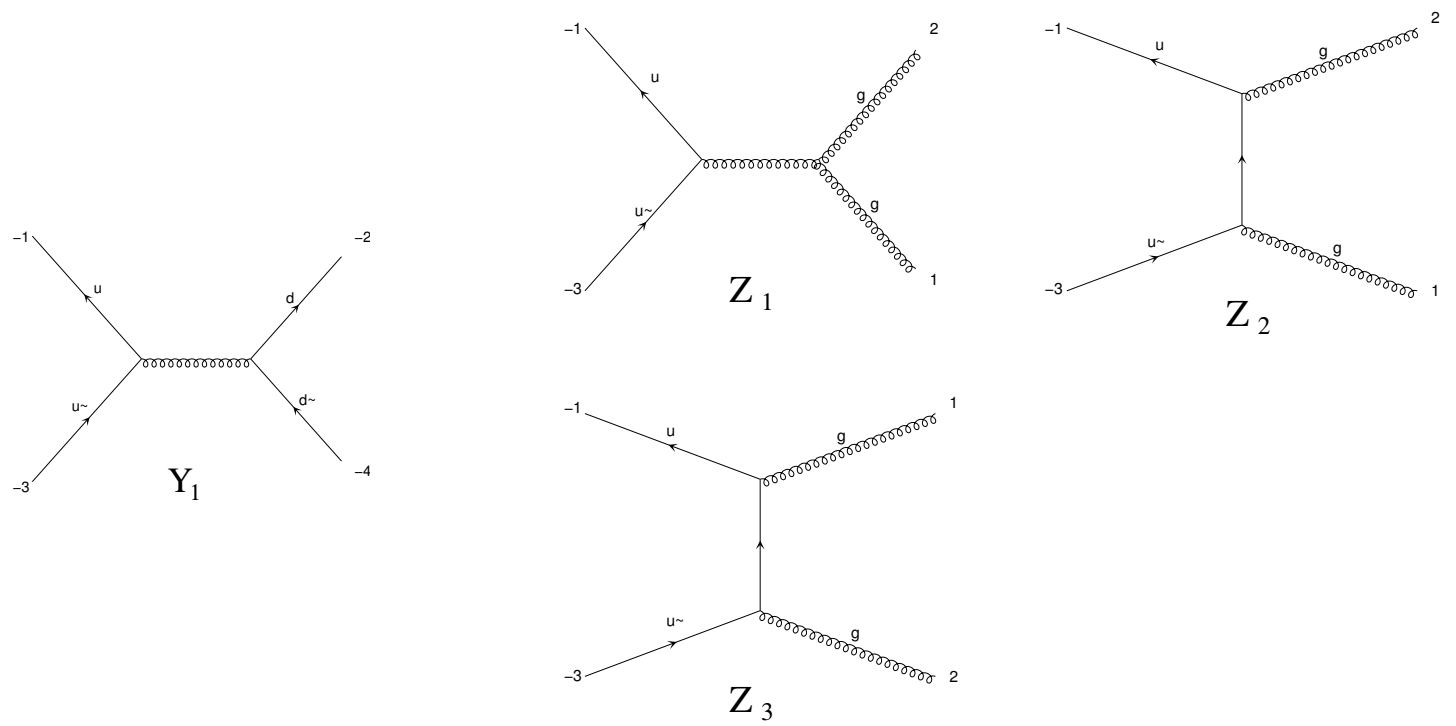

Figure 2. Diagrams that contribute to $0 \rightarrow u \bar{u} d \bar{d}$ and $0 \rightarrow u \bar{u} g g$.

whose contributing Feynman diagrams are depicted in figure 2. The amplitude that corresponds to eq. (C.15) is:

$$
\mathcal{Y}=\sum_{i=1}^{2} \Lambda\left(\gamma_{i}^{(Y)}\right) \widehat{\mathcal{Y}}\left(\gamma_{i}^{(Y)}\right)
$$

with:

$$
\begin{aligned}
\gamma_{1}^{(Y)} & =(-1 ;-4)(-2 ;-3), & \gamma_{2}^{(Y)} & =(-1 ;-3)(-2 ;-4) \\
\Lambda\left(\gamma_{1}^{(Y)}\right) & =\delta_{a_{-1} a_{-4}} \delta_{a_{-2} a_{-3}}, & \Lambda\left(\gamma_{2}^{(Y)}\right) & =\frac{1}{N} \delta_{a_{-1} a_{-3}} \delta_{a_{-2} a_{-4}}, \\
2 \widehat{\mathcal{Y}}\left(\gamma_{1}^{(Y)}\right) & =Y_{1}, & 2 \widehat{\mathcal{Y}}\left(\gamma_{2}^{(Y)}\right) & =-Y_{1} .
\end{aligned}
$$

Hence, the colour-flow matrix:

$$
C_{i j}^{(Y)} \equiv C\left(\gamma_{i}^{(Y)}, \gamma_{j}^{(Y)}\right)
$$

reads:

$$
\begin{aligned}
& C_{11}^{(Y)}=N^{2}, \\
& C_{12}^{(Y)}=C_{22}^{(Y)}=1,
\end{aligned}
$$

consistently with the fact that $p\left(\gamma_{1}^{(Y)}, \gamma_{1}^{(Y)}\right)=2$, and $p\left(\gamma_{1}^{(Y)}, \gamma_{2}^{(Y)}\right)=p\left(\gamma_{2}^{(Y)}, \gamma_{2}^{(Y)}\right)=0$.

In the case of the process of eq. (C.16) the amplitude reads:

$$
\mathcal{Z}=\sum_{i=1}^{2} \Lambda\left(\gamma_{i}^{(Z)}\right) \widehat{\mathcal{Z}}\left(\gamma_{i}^{(Z)}\right),
$$

with:

$$
\begin{aligned}
\gamma_{1}^{(Z)} & =(-1 ; 1,2 ;-3), & \gamma_{2}^{(Z)} & =(-1 ; 2,1 ;-3) \\
\Lambda\left(\gamma_{1}^{(Z)}\right) & =\left(\lambda^{a_{1}} \lambda^{a_{2}}\right)_{a_{-1} a_{-3}}, & \Lambda\left(\gamma_{2}^{(Z)}\right) & =\left(\lambda^{a_{2}} \lambda^{a_{1}}\right)_{a_{-1} a_{-3}} \\
\widehat{\mathcal{Z}}\left(\gamma_{1}^{(Z)}\right) & =Z_{2}-i Z_{1}, & \widehat{\mathcal{Z}}\left(\gamma_{2}^{(Z)}\right) & =Z_{3}+i Z_{1}
\end{aligned}
$$


Hence, the colour-flow matrix:

$$
C_{i j}^{(Z)} \equiv C\left(\gamma_{i}^{(Z)}, \gamma_{j}^{(Z)}\right)
$$

reads:

$$
\begin{aligned}
& C_{11}^{(Z)}=C_{22}^{(Z)}=N^{3} / 4-N / 2+1 /(4 N), \\
& C_{12}^{(Z)}=-N / 4+1 /(4 N),
\end{aligned}
$$

consistently with the fact that $p\left(\gamma_{1}^{(Z)}, \gamma_{1}^{(Z)}\right)=p\left(\gamma_{2}^{(Z)}, \gamma_{2}^{(Z)}\right)=3$ and $p\left(\gamma_{1}^{(Z)}, \gamma_{2}^{(Z)}\right)=1$.

Soft limit, fixed real flows. This is given in eq. (3.105). I list below the result for each closed flow, omitting the factors $-g_{S}^{2} C_{i j}^{(X)}$. One obtains: ${ }^{9}$

$$
\begin{array}{lll}
\left(\Gamma_{1}, \Gamma_{1}\right) & \longrightarrow & -2[-2,-3] \widehat{\mathcal{Y}}\left(\gamma_{1}^{(Y)}\right)^{\star} \widehat{\mathcal{Y}}\left(\gamma_{1}^{(Y)}\right), \\
\left(\Gamma_{2}, \Gamma_{2}\right) & \longrightarrow & -2[-1,-4] \widehat{\mathcal{Y}}\left(\gamma_{1}^{(Y)}\right)^{\star} \widehat{\mathcal{Y}}\left(\gamma_{1}^{(Y)}\right), \\
\left(\Gamma_{1}, \Gamma_{3}\right) & \longrightarrow & (-[-2,-4]-[-2,-3]+[-3,-4]) \widehat{\mathcal{Y}}\left(\gamma_{1}^{(Y)}\right)^{\star} \widehat{\mathcal{Y}}\left(\gamma_{2}^{(Y)}\right), \\
\left(\Gamma_{1}, \Gamma_{4}\right) & \longrightarrow & ([-1,-2]-[-2,-3]-[-1,-3]) \widehat{\mathcal{Y}}\left(\gamma_{1}^{(Y)}\right)^{\star} \widehat{\mathcal{Y}}\left(\gamma_{2}^{(Y)}\right), \\
\left(\Gamma_{2}, \Gamma_{3}\right) & \longrightarrow & ([-1,-2]-[-1,-4]-[-2,-4]) \widehat{\mathcal{Y}}\left(\gamma_{1}^{(Y)}\right)^{\star} \widehat{\mathcal{Y}}\left(\gamma_{2}^{(Y)}\right), \\
\left(\Gamma_{2}, \Gamma_{4}\right) & \longrightarrow & (-[-1,-3]-[-1,-4]+[-3,-4]) \widehat{\mathcal{Y}}\left(\gamma_{1}^{(Y)}\right)^{\star} \widehat{\mathcal{Y}}\left(\gamma_{2}^{(Y)}\right), \\
\left(\Gamma_{3}, \Gamma_{3}\right) & \longrightarrow & -2[-2,-4] \widehat{\mathcal{Y}}\left(\gamma_{2}^{(Y)}\right)^{\star} \widehat{\mathcal{Y}}\left(\gamma_{2}^{(Y)}\right), \\
\left(\Gamma_{4}, \Gamma_{4}\right) & \longrightarrow & -2[-1,-3] \widehat{\mathcal{Y}}\left(\gamma_{2}^{(Y)}\right)^{\star} \widehat{\mathcal{Y}}\left(\gamma_{2}^{(Y)}\right),
\end{array}
$$

where the eikonal factor $[k, l]$ has been introduced in eq. (2.31). According to eqs. (C.10)(C.13), at the leading colour only eq. (C.31) and (C.32) contribute to the soft limit, the other closed flows being colour-suppressed (or identically equal to zero).

Collinear limit $\boldsymbol{g} \| \boldsymbol{d}$, fixed real flows. This is given in eq. (3.109). In that equation, the kernels are flow-independent; hence, the only flow-specific information is contained in $\delta\left(\Gamma^{\prime}, \Gamma\right)$, and in the reduced matrix elements. Using the definition of the former, given in eq. (3.111), it is easy to obtain the following list of closed flows which give a non-zero contribution in the collinear limit:

$$
\begin{aligned}
& \left(\Gamma_{1}, \Gamma_{1}\right) \longrightarrow \hat{\mathcal{Y}}\left(\gamma_{1}^{(Y)}\right)^{\star} \widehat{\mathcal{Y}}\left(\gamma_{1}^{(Y)}\right), \\
& \left(\Gamma_{1}, \Gamma_{3}\right) \longrightarrow \hat{\mathcal{Y}}\left(\gamma_{1}^{(Y)}\right)^{\star} \widehat{\mathcal{Y}}\left(\gamma_{2}^{(Y)}\right), \\
& \left(\Gamma_{3}, \Gamma_{3}\right) \longrightarrow \hat{\mathcal{Y}}\left(\gamma_{2}^{(Y)}\right)^{\star} \hat{\mathcal{Y}}\left(\gamma_{2}^{(Y)}\right),
\end{aligned}
$$

where I have also indicated the reduced amplitudes that enter the limit of each closed-flow amplitude squared. Equation (C.39) is the only leading-colour contribution. As was already discussed at length in the main text, eqs. (C.31)-(C.38) are consistent with eqs. (C.39)(C.41) in the sense of eq. (2.29). Here, the reader can check with an explicit example what has been claimed after eq. (2.113) (which applies identically to the case of quark-gluon amplitudes). Namely, that despite the fact that individual eikonal factors may diverge

\footnotetext{
${ }^{9}$ Because of eq. (2.24), I shall consider here explicitly only closed flows $\left(\Gamma_{i}, \Gamma_{j}\right)$ with $i \leq j$.
} 
in a given collinear limit, the corresponding interferences of amplitudes may still be nondivergent in that limit. This is the case here for eqs. (C.34) and (C.35), which do not have a $g \| d$ collinear divergence owing to the cancellations that occur among pairs of eikonals which contain the momentum of $d$, and differ by the sign in front of them.

Collinear limit $\boldsymbol{d} \| \overline{\boldsymbol{d}}$, fixed real flows. This is given in eq. (3.156). Also in this case, the kernels are flow-independent; the only flow-specific information one needs to derive is which closed flows give a non-zero contribution, and the reduced matrix elements they are associated with. In order to do this, one first determines to which class of flows the real-emission ones belong. Using the definitions given in eqs. (3.145) and (3.147), one obtains:

$$
\begin{aligned}
\Gamma_{1}, \Gamma_{2} & \in \mathcal{F}^{(J)}, \\
\Gamma_{4} & \in \mathcal{F}^{(K)}, \\
\Gamma_{3} & \notin \mathcal{F}^{(J)} \cup \mathcal{F}^{(K)} .
\end{aligned}
$$

Using the definitions of the $J$ and $K$ operators, eqs. (3.134) and (3.135), it is easy to see that:

$$
\begin{aligned}
J \gamma_{1}^{(Z)} & =\Gamma_{2}, & J \gamma_{2}^{(Z)} & =\Gamma_{1}, \\
K \gamma_{1}^{(Z)} & =\Gamma_{4}, & K \gamma_{2}^{(Z)} & =\Gamma_{4} .
\end{aligned}
$$

Hence, from eqs. (3.162)-(3.165):

$$
\begin{array}{lll}
\left(\Gamma_{1}, \Gamma_{1}\right) & \longrightarrow & \widehat{\mathcal{Z}}\left(\gamma_{2}^{(Z)}\right)^{\star} \widehat{\mathcal{Z}}\left(\gamma_{2}^{(Z)}\right), \\
\left(\Gamma_{2}, \Gamma_{2}\right) & \longrightarrow & \widehat{\mathcal{Z}}\left(\gamma_{1}^{(Z)}\right)^{\star} \widehat{\mathcal{Z}}\left(\gamma_{1}^{(Z)}\right), \\
\left(\Gamma_{1}, \Gamma_{4}\right) & \longrightarrow & -\widehat{\mathcal{Z}}\left(\gamma_{2}^{(Z)}\right)^{\star}\left(\widehat{\mathcal{Z}}\left(\gamma_{1}^{(Z)}\right)+\widehat{\mathcal{Z}}\left(\gamma_{2}^{(Z)}\right)\right) \\
\left(\Gamma_{2}, \Gamma_{4}\right) & \longrightarrow & -\widehat{\mathcal{Z}}\left(\gamma_{1}^{(Z)}\right)^{\star}\left(\widehat{\mathcal{Z}}\left(\gamma_{1}^{(Z)}\right)+\widehat{\mathcal{Z}}\left(\gamma_{2}^{(Z)}\right)\right) \\
\left(\Gamma_{4}, \Gamma_{4}\right) & \longrightarrow & \left(\widehat{\mathcal{Z}}\left(\gamma_{1}^{(Z)}\right)+\widehat{\mathcal{Z}}\left(\gamma_{2}^{(Z)}\right)\right)^{\star}\left(\widehat{\mathcal{Z}}\left(\gamma_{1}^{(Z)}\right)+\widehat{\mathcal{Z}}\left(\gamma_{2}^{(Z)}\right)\right)
\end{array}
$$

where only eqs. (C.47) and (C.48) contribute at the leading colour.

Soft limit, fixed Born flows. One starts by constructing the linear combinations of matrix elements with well-defined limits, which is equivalent to determining the sets defined in eq. (3.116). Using eq. (C.3), (C.4), and (C.18), one obtains:

$$
\begin{aligned}
& \xi\left(\gamma_{1}^{(Y)}\right)=\left\{\Gamma_{1}, \Gamma_{2}\right\}, \\
& \xi\left(\gamma_{2}^{(Y)}\right)=\left\{\Gamma_{3}, \Gamma_{4}\right\} .
\end{aligned}
$$

Therefore, the relevant matrix elements will be (see eq. (3.114)):

$$
\begin{aligned}
& \mathcal{M}\left(\gamma_{1}^{(Y)}, \gamma_{1}^{(Y)}\right)=\mathcal{M}\left(\Gamma_{1}, \Gamma_{1}\right)+\mathcal{M}\left(\Gamma_{2}, \Gamma_{2}\right) \\
& \mathcal{M}\left(\gamma_{1}^{(Y)}, \gamma_{2}^{(Y)}\right)=\mathcal{M}\left(\Gamma_{1}, \Gamma_{3}\right)+\mathcal{M}\left(\Gamma_{1}, \Gamma_{4}\right)+\mathcal{M}\left(\Gamma_{2}, \Gamma_{3}\right)+\mathcal{M}\left(\Gamma_{2}, \Gamma_{4}\right) \\
& \mathcal{M}\left(\gamma_{2}^{(Y)}, \gamma_{2}^{(Y)}\right)=\mathcal{M}\left(\Gamma_{3}, \Gamma_{3}\right)+\mathcal{M}\left(\Gamma_{4}, \Gamma_{4}\right)
\end{aligned}
$$


where I have exploited the fact that $\mathcal{M}\left(\Gamma_{1}, \Gamma_{2}\right)=0, \mathcal{M}\left(\Gamma_{3}, \Gamma_{4}\right)=0$ owing to eq. (C.11). According to eqs. (C.22) and (C.23), only eq. (C.54) will give a leading-colour contribution. The soft limits of these quantities can be computed using eqs. eq. (3.86) and (3.115). One obtains:

$$
\begin{array}{rlr}
\left(\gamma_{1}^{(Y)}, \gamma_{1}^{(Y)}\right) & \longrightarrow & -L C([-1,-4]+[-2,-3]), \\
\left(\gamma_{1}^{(Y)}, \gamma_{2}^{(Y)}\right) & \longrightarrow & -S C([-1,-2]-[-1,-3]-[-1,-4] \\
& \longrightarrow & -[-2,-3]-[-2,-4]+[-3,-4]), \\
\left(\gamma_{2}^{(Y)}, \gamma_{2}^{(Y)}\right) & \longrightarrow & -S C([-1,-3]+[-2,-4]),
\end{array}
$$

where I have omitted overall terms $g_{S}^{2} \widehat{\mathcal{Y}}\left(\gamma_{i}^{(Y)}\right)^{\star} \widehat{\mathcal{Y}}\left(\gamma_{j}^{(Y)}\right)$, and $L C$ and $S C$ are given in eq. (C.13). It is immediate to see that the results of eqs. (C.57)-(C.59) are consistent with those of eqs. (C.31)-(C.38). It should also be stressed that the fact that a single colour factor factorizes in eqs. (C.57)-(C.59) is accidental, and due to the relative simplicity of the process being considered here. In general, at fixed Born flows different (combinations of) eikonals will be multiplied by different colour factors; the only way in which the colour fully factorizes in the infrared limits is by fixing real flows.

Collinear limit $\boldsymbol{g} \| \boldsymbol{d}$, fixed Born flows. As one can see from eqs. (3.120)-(3.122), this case is trivial: the same closed flow appears on both sides of eq. (3.120). Note that, while all matrix elements in eqs. (C.54)-(C.56) will be collinear divergent, this behaviour is driven by the first term on each of the r.h.s.'s of these equations - this has been discussed in general in the main text (see e.g. eq. (3.123) for quark-gluon amplitudes).

Collinear limit $\boldsymbol{d} \| \overline{\boldsymbol{d}}$, fixed Born flows. As was discussed in section 3.3.3, one cannot treat a $g \rightarrow q \bar{q}$ branching by fixing the Born flows. The present example allows one to further this point. The problem arises from the fact that the $K$ operator has a multivalued inverse, see eq. (C.46) - it therefore becomes ambiguous whether to associate the contribution due to $\Gamma_{4}$ with that of $\gamma_{1}^{(Z)}=K_{1}^{-1} \Gamma_{4}$ or that of $\gamma_{2}^{(Z)}=K_{2}^{-1} \Gamma_{4}$. To proceed, one must look at the diagrammatic level. From eq. (C.8) and figure 1, one sees that diagrams $X_{4}$ and $X_{5}$, that contribute to $\widehat{\mathcal{X}}\left(\Gamma_{4}\right)$, correspond in the collinear limit to diagrams $Z_{2}$ and $Z_{3}$ of figure 2 , respectively. Now, $Z_{2}$ enters $\widehat{\mathcal{Z}}\left(\gamma_{1}^{(Z)}\right)$, and $Z_{3}$ enters $\widehat{\mathcal{Z}}\left(\gamma_{2}^{(Z)}\right)$ (see eq. (C.27)). Therefore, in order to match the collinear behaviour, the $X_{4}$ and $X_{5}$ parts of $\widehat{\mathcal{X}}\left(\Gamma_{4}\right)$ should be assigned to $\widehat{\mathcal{Z}}\left(\gamma_{1}^{(Z)}\right)$ and $\widehat{\mathcal{Z}}\left(\gamma_{2}^{(Z)}\right)$ respectively, which is not a gauge-invariant procedure.

Open Access. This article is distributed under the terms of the Creative Commons Attribution Noncommercial License which permits any noncommercial use, distribution, and reproduction in any medium, provided the original author(s) and source are credited.

\section{References}

[1] M.L. Mangano and S.J. Parke, Multi-parton amplitudes in gauge theories, Phys. Rept. 200 (1991) 301 [hep-th/0509223] [SPIRES]. 
[2] F.A. Berends and W.T. Giele, Recursive calculations for processes with $n$ gluons, Nucl. Phys. B 306 (1988) 759 [SPIRES].

[3] F. Cachazo, P. Svrček and E. Witten, MHV vertices and tree amplitudes in gauge theory, JHEP 09 (2004) 006 [hep-th/0403047] [SPIRES].

[4] R. Britto, F. Cachazo and B. Feng, New recursion relations for tree amplitudes of gluons, Nucl. Phys. B 715 (2005) 499 [hep-th/0412308] [SPIRES].

[5] F. Caravaglios, M.L. Mangano, M. Moretti and R. Pittau, A new approach to multi-jet calculations in hadron collisions, Nucl. Phys. B 539 (1999) 215 [hep-ph/9807570] [SPIRES].

[6] P.D. Draggiotis, R.H.P. Kleiss and C.G. Papadopoulos, Multi-jet production in hadron collisions, Eur. Phys. J. C 24 (2002) 447 [hep-ph/0202201] [SPIRES].

[7] F. Caravaglios and M. Moretti, An algorithm to compute Born scattering amplitudes without Feynman graphs, Phys. Lett. B 358 (1995) 332 [hep-ph/9507237] [SPIRES].

[8] C. Duhr, S. Hoeche and F. Maltoni, Color-dressed recursive relations for multi-parton amplitudes, JHEP 08 (2006) 062 [hep-ph/0607057] [SPIRES].

[9] T. Gleisberg and F. Krauss, Automating dipole subtraction for QCD NLO calculations, Eur. Phys. J. C 53 (2008) 501 [arXiv:0709.2881] [SPIRES].

[10] G. Bevilacqua, M. Czakon, C.G. Papadopoulos, R. Pittau and M. Worek, Assault on the $N L O$ wishlist: $p p \rightarrow t \bar{t} b \bar{b}, J H E P 09$ (2009) 109 [arXiv:0907.4723] [SPIRES].

[11] R. Frederix, S. Frixione, F. Maltoni and T. Stelzer, Automation of next-to-leading order computations in QCD: the FKS subtraction, JHEP 10 (2009) 003 [arXiv:0908.4272] [SPIRES].

[12] R. Frederix, T. Gehrmann and N. Greiner, Integrated dipoles with MadDipole in the MadGraph framework, JHEP 06 (2010) 086 [arXiv:1004.2905] [SPIRES].

[13] S. Frixione, Z. Kunszt and A. Signer, Three jet cross-sections to next-to-leading order, Nucl. Phys. B 467 (1996) 399 [hep-ph/9512328] [SPIRES].

[14] S. Frixione, A general approach to jet cross-sections in QCD, Nucl. Phys. B 507 (1997) 295 [hep-ph/9706545] [SPIRES].

[15] S. Catani and M.H. Seymour, A general algorithm for calculating jet cross sections in NLO QCD, Nucl. Phys. B 485 (1997) 291 [Erratum ibid. B 510 (1998) 503] [hep-ph/9605323] [SPIRES].

[16] R.K. Ellis, W.T. Giele, Z. Kunszt, K. Melnikov and G. Zanderighi, One-loop amplitudes for $W+3$ jet production in hadron collisions, JHEP 01 (2009) 012 [arXiv:0810.2762] [SPIRES].

[17] A. van Hameren, C.G. Papadopoulos and R. Pittau, Automated one-loop calculations: a proof of concept, JHEP 09 (2009) 106 [arXiv:0903.4665] [SPIRES].

[18] C.F. Berger et al., Precise predictions for $W+4$ jet production at the Large Hadron Collider, Phys. Rev. Lett. 106 (2011) 092001 [arXiv:1009.2338] [SPIRES].

[19] P. Mastrolia, G. Ossola, T. Reiter and F. Tramontano, Scattering amplitudes from unitarity-based reduction algorithm at the integrand-level, JHEP 08 (2010) 080 [arXiv : 1006.0710] [SPIRES]. 
[20] V. Hirschi et al., Automation of one-loop QCD corrections, JHEP 05 (2011) 044 [arXiv: 1103.0621] [SPIRES].

[21] Z. Bern, L.J. Dixon, D.C. Dunbar and D.A. Kosower, One-loop n-point gauge theory amplitudes, unitarity and collinear limits, Nucl. Phys. B 425 (1994) 217 [hep-ph/9403226] [SPIRES].

[22] R.K. Ellis, W.T. Giele and Z. Kunszt, A numerical unitarity formalism for evaluating one-loop amplitudes, JHEP 03 (2008) 003 [arXiv:0708.2398] [SPIRES].

[23] R.K. Ellis, W.T. Giele, Z. Kunszt and K. Melnikov, Masses, fermions and generalized D-dimensional unitarity, Nucl. Phys. B 822 (2009) 270 [arXiv:0806.3467] [SPIRES].

[24] F. del Aguila and R. Pittau, Recursive numerical calculus of one-loop tensor integrals, JHEP 07 (2004) 017 [hep-ph/0404120] [SPIRES].

[25] G. Ossola, C.G. Papadopoulos and R. Pittau, Reducing full one-loop amplitudes to scalar integrals at the integrand level, Nucl. Phys. B 763 (2007) 147 [hep-ph/0609007] [SPIRES].

[26] Z. Bern, L.J. Dixon and D.A. Kosower, Progress in one-loop QCD computations, Ann. Rev. Nucl. Part. Sci. 46 (1996) 109 [hep-ph/9602280] [SPIRES].

[27] D.A. Kosower, Antenna factorization of gauge-theory amplitudes, Phys. Rev. D 57 (1998) 5410 [hep-ph/9710213] [SPIRES].

[28] W.T. Giele and E.W.N. Glover, Higher order corrections to jet cross-sections in $e^{+} e^{-}$ annihilation, Phys. Rev. D 46 (1992) 1980 [SPIRES].

[29] J.M. Campbell, M.A. Cullen and E.W.N. Glover, Four jet event shapes in electron positron annihilation, Eur. Phys. J. C 9 (1999) 245 [hep-ph/9809429] [SPIRES].

[30] K. Odagiri, Color connection structure of supersymmetric QCD $(2 \rightarrow 2)$ processes, JHEP 10 (1998) 006 [hep-ph/9806531] [SPIRES].

[31] J. Alwall, M. Herquet, F. Maltoni, O. Mattelaer and T. Stelzer, MadGraph 5: going beyond, to appear, https://launchpad.net/madgraph5.

[32] G. Abelof and A. Gehrmann-De Ridder, Antenna subtraction for the production of heavy particles at hadron colliders, JHEP 04 (2011) 063 [arXiv:1102.2443] [SPIRES].

[33] Z. Nagy and D.E. Soper, Parton showers with quantum interference, JHEP 09 (2007) 114 [arXiv:0706.0017] [SPIRES].

[34] C.H. Chung, M. Krämer and T. Robens, An alternative subtraction scheme for next-to-leading order QCD calculations, JHEP 06 (2011) 144 [arXiv:1012.4948] [SPIRES].

[35] S. Frixione and M. Grazzini, Subtraction at NNLO, JHEP 06 (2005) 010 [hep-ph/0411399] [SPIRES]. 